\title{
THERMALLY DRIVEN CIRCULATION
}

\author{
by \\ Haim Nelken \\ B.A., Physics and Mathematics \\ Hebrew University, Jerusalem, Israel \\ 1981 \\ S.M., Oceanography \\ Massachusetts Institute of Technology \\ 1984 \\ Submitted in partial fulfillment of the \\ requirements for the degree of \\ Doctor of Science \\ at the \\ Massachusetts Institute of Technology \\ and the \\ Woods Hole Oceanographic Institution \\ August, 1987 \\ (C)Haim Nelken 1987
}

The author hereby grants to M.I.T. permission to reproduce and to distribute copies of this thesis in whole or in part.

Signature of the author

Joint Program in Oceanography, Massachusetts Institute of Technology-

Woods Hole Oceanographic Institution, August 1987.

Certified by

\section{Thesis supervisor}

Accepted by

Chairhan, Joint Committee for Physical Oceanography. Massachusetts Institute of Technology-

Woods Hole Oceanographic Institution. 


\title{
THERMALLY DRIVEN CIRCULATION
}

\author{
by
}

\author{
Haim Nelken
}

\begin{abstract}
Submitted to the Massachusetts Institute of Technology-Woods Hole Oceanographic Institution, Joint Program, in August, 1987, in partial fulfillment of the requirements for the degree of Doctor of Philosophy.
\end{abstract}

\begin{abstract}
Several problems connected by the theme of thermal forcing are addressed herein. The main topic is the stratification and flow field resulting from imposing a specified heat flux on a fluid that is otherwise confined to a rigid insulating basin. In addition to the traditional eddy viscosity and diffusivity, turbulent processes are also included by a convective overturning adjustment at locations where the local density field is unstable.

Two classes of problems are treated. The first is the large scale meridional pattern of a fluid in an annulus. The detailed treatment is carried out in two steps. In the beginning (chapter 2) it is assumed that the fluid is very diffusive, hence, to first approximation no flow field is present. It is found that the convective overturning adjustment changes the character of the stratification in all the regions that are cooled from the top, resulting in a temperature field that is nearly depth independent in the northernmost latitudes. The response to a seasonal cycle in the forcing, and the differences between averaging the results from the end of each season compared to driving the fluid by a mean forcing are analyzed. In particular, the resulting sea surface temperature is warmer in the former procedure. This observation is important in models where the heat flux is sensitive to the gradient of air to sea surface temperatures.

The analysis of the problem continues in chapter 5 where the contribution of the flow field is included in the same configuration. The dimensionless parameter controlling the circulation is now the Rayleigh number, which is a measure of the relative importance of gravitational and viscous forces. The effects of the convective overturning adjustment is investigated at different Rayleigh numbers. It is shown that not only is the stratification now always stable, but also that the vigorous vertical mixing reduces the effective Rayleigh number; thereby the flow field is more moderate, the thermocline deepens, and the horizontal surface temperature gradients are weaker. The interior of the fluid is colder compared to cases without convective overturning, and, because the amount of heat in the system is assumed to be fixed, the surface temperature is warmer.

The fluid is not only forced by a mean heat flux, or a seasonally varying one, but its behavior under permanent winter and summer conditions is also investigated. A steady state for the experiments where the net heat flux does not vanish is defined as that state where the flow field and temperature structure are not changing with time except for an almost uniform temperature decrease or increase everywhere. It is found
\end{abstract}


that when winter conditions prevail the circulation is very strong, while it is rather weak for continuous summer forcing. In contrast to those results, if a yearly cycle is imposed, the circulation tends to reach a minimum in the winter time and a maximum in the summer. This suggests that, depending on the Rayleigh number, there is a phase leg of several months between the response of the ocean and the imposed forcing.

Differences between the two averaging procedures mentioned before are also observed when the flow field is present, especially for large Rayleigh numbers. The circulation is found to be weaker and the sea surface temperature colder in the mean of the seasonal realizations compared to the steady state derived by the mean forcing.

As an extension to the numerical results, an analytic model is presented in chapter 4 for a similar annular configuration. The assumed dynamics is a bit different, with a mixed layer on top of a potential vorticity conserving interior. It is demonstrated that the addition of the thermal wind balance to the conservation of potential vorticity in the axially symmetric problem leads to the result that typical fluid trajectories in the interior are straight lines pointing downward going north to south. The passage of information in the system is surprisingly in the opposite sense to the clockwise direction of the flow.

A model for water mass formation by buoyancy loss in the absence of a flow field is introduced in chapter 3. The idea behind it is to use the turbulent mixing parameterization to generate chimney-like structures in open water, followed by alongisopycnal advection and diffusion. This model can be applied to many observations of mode water. In particular, in this work it is related to the chimneys observed by the MEDOC Group (1970), and the Levantine Intermediate Water in the Eastern Mediterranean Basin. An analytic prediction of the depth of the water mass is derived and depends on the forcing and initial stratification. It suggests that the depth of shallow mode water like the $18^{\circ} \mathrm{C}$ water or the Levantine Intermediate Water would not be very sensitive to reasonable changes in atmospheric forcing. Similar conclusions were also reached by Warren (1972) by assuming that the temperature in the thermocline decreases linearly with depth, and by approximating the energy balance in a water column by a Newtonian cooling law.

Thesis supervisor: Glenn R. Flierl

Professor of Physical Oceanography 


\section{Acknowledgments}

I am grateful to Professor Glenn Flierl for his patience and steady guidance throughout this work.

This thesis also benefited from commentary made by Doctors Harry Bryden, Jim Luyten, Dave Musgrave, and Bill Young - all of whom are associated with my Advisory Committee-and Doctor Mike McCartney, who was the chairman of the defense.

It is dedicated with love to four persons: my grandfather, mother, father, and Tami. 


\section{Table of contents}

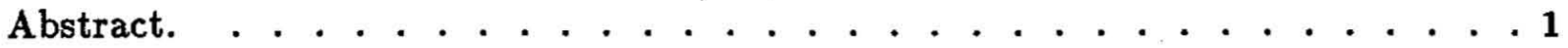

Acknowledgments. . . . . . . . . . . . . . 3

Table of contents. . . . . . . . . . . . . . . . 5

List of figures. . . . . . . . . . . . . . . . 7

Chapter 1: Preliminaries

1. Literature survey. . . . . . . . . . . . . . . . . . . 10

2. Introduction. . . . . . . . . . . . . . 21

3. Governing equations. . . . . . . . . . . . . 26

Chapter 2: The effects of heating in the absence of a flow field

1. Introduction. . . . . . . . . . . . . . . . . . . . 32

2. Heat equation. . . . . . . . . . . . . . . . . . 33

3. Convective overturning. . . . . . . . . . . . . . . . . . . 36

4. Numerical implementation. . . . . . . . . . . . . . . . . . 42

5. Results.

a. Vertical mixing with specified fixed flux. . . . . . . . 45

b. Forcing with a yearly cycle. . . . . . . . . . . . . . . 48

c. Flux depending on air to sea surface temperature gradient. . . . 51

d. Different averaging schemes. . . . . . . . . . . . . 57

6. Discussion. . . . . . . . . . . . . . . . . . . . . . . . . . . 59

a. Flux versus air to surface temperature gradient. . . . . . . 60

b. Steady state versus average of periodic states. . . . . . . . . 63

7. Conclusions. . . . . . . . . . . . . . . . . . . . . . 66

Chapter 3: Water mass formation by buoyancy loss without advection

1. Introduction. . . . . . . . . . . . . . 68

2. The model. . . . . . . . . . . . . . . . . . . . . . . . . . . 71

3. Numerical implementation. . . . . . . . . . . . . . . . . . . 73

4. Results. . . . . . . . . . . . . . . . . . . . . . 74

5. Discussion. . . . . . . . . . . . . . . . . . . . . . . . . . . 82

6. Relation to observations. . . . . . . . . . . . . . . . . . . . . 87

7. Conclusions. . . . . . . . . . . . . . . . . . . . . . . . . . . 97

Chapter 4: Two region vertical circulation driven by cooling

1. Introduction. . . . . . . . . . . . . . . . . . . . . 93

2. The model. . . . . . . . . . . . . . . . . . . . . . . . . . . 95

3. Upper layer balances. . . . . . . . . . . . . . . . . . . . . . . . 98

a. Heat equation. . . . . . . . . . . . . . . . . . . . . . . . . . 98

b. Momentum balance. . . . . . . . . . . . . . . . . . . 100

4. Lower layer balances. . . . . . . . . . . . . . . . . . . . . . . 101

a. Heat budget. . . . . . . . . . . . . . . . . . . . . . . . 101

b. Momentum balance. . . . . . . . . . . . . . . . . . . . . 101

5. A more general model for the mixed layer. . . . . . . . . . . . 102 
6. The uncoupled problem.

a. Analysis of heat budget in the mixed layer. . . . . . . . . 105

b. Momentum budget and solutions for the upper layer. . . . . . . 107

c. Lower layer conservation constraints. . . . . . . . . . . . . . 109

1. The potential vorticity. . . . . . . . . . . . . . . 110

II. Non-dissipative fluid. . . . . . . . . . . . . . . . . . . 111

III. The beta effect. . . . . . . . . . . . . . . . . . . 114

IV. Additional results for angular momentum conserving flow. . . 115

d. Effects of dissipation on the fluid trajectories. . . . . . . . 117

7. Discussion of the coupled problem. . . . . . . . . . . 121

a. Interior constraints. . . . . . . . . . . . . . . . 122

8. Conclusions. . . . . . . . . . . . . . . . . . . . . 124

Chapter 5: Effects of mixing and seasonal cycle on differentially heated flows

1. Introduction. . . . . . . . . . . . . . . . . . 126

2. Circulation in the absence of rotation. . . . . . . . . . . . 128

3. Simple analysis of the governing equations. . . . . . . . . . . 130

4. Parameter range. . . . . . . . . . . . . . . . . . . . . . . . 137

a. Standard case, no mixing. . . . . . . . . . . . . . 137

b. Mixing case. . . . . . . . . . . . . . . . . . . . . 138

c. Forcing. . . . . . . . . . . . . . . . . . . . . . . . 138

5. Numerical Scheme. . . . . . . . . . . . . . . . . 140

6. Results. . . . . . . . . . . . . . . . . . . . 141

7. Constant heat flux.

a. No mixing. . . . . . . . . . . . . . . . . . . . 142

b. Mixing. . . . . . . . . . . . . . . . . . 145

8. Discussion. . . . . . . . . . . . . . . . . . . . . . . 146

9. The heat equation and its leading order balance. . . . . . . . 148

10. Variable heat flux. . . . . . . . . . . . . 153

11. Discussion. . . . . . . . . . . . . . 165

12. Conclusions. . . . . . . . . . . . . . 173

Concluding remarks. . . . . . . . . . . . . . . . . 175

Appendix: Evaluation of the Arakawa Jacobian in a uniform grid. . . . . . 177

References. . . . . . . . . . . . . . 181 


\section{List of figures}

(2.1). A sketch of the interface between the convective overturning region and the stable one. . . . . . . . . . . . . . . . . . . . . . . . . . . . . 40 40

(2.2). Temperature structure from solving equation (2.4) for a specified $\cos (\pi y)$ heat flux. The ratio $\kappa$ between the diffusivities in the northern $\left(\kappa_{2}\right)$ and southern $\left(\kappa_{1}\right)$ regions is 1 in figure (a) and 100 elsewhere. (a) $\kappa=\kappa_{1}=1$, as in the analytic solution (2.2) with no overturning. (b) $\kappa_{1}=1$, regions to the right of the heavy line are weakly unstable. (c) $\kappa_{1}=10$. (d) $\kappa_{1}=0.1$. . . . . . . . . . . . . 46

(2.3). Temperature structure in a periodic steady state based on monthly varying heat flux taken from Oort and Vonder Haar (1976). (a) December. (b) February. (c) May. (d) August. (e) October. . . . . . . . . . . . . . . . . . . . . 49

(2.4). The relation between the $T^{\mathrm{air}}$ and $T^{\mathrm{sur}}$ when only the first is given and the flux is proportional to the difference between them. Line (A), the air temperature. Line (B), the initial surface temperature. Line (C), the surface temperature after some time. Line (D), the final surface temperature. (b) is an enlargement of (a) around the intersection of the four lines. . . . . . . . . . . . . . . . . . . . 53

(2.5). Temperature structure when the flux depends on the specified air temperature. (b) is the same as (a) but after running the model 50 times longer. . . . . 54

(2.6). South-north temperature cross sections at three different depth. Line (A), surface temperature. Line (B), $13 \%$ of the depth from the surface. Line (C), $7 \%$ of the depth from the bottom. (a) Corresponds to figure (2.1)b. (b) Based on data taken from figure (2.5)b. Note the different vertical scales. . . . . . . . 56

(2.7). Temperature structure for different averages with monthly data taken from Oort and Vonder Haar (1976). (a) Average of twelve monthly realizations, each with $\kappa=100$. (b) A steady state from average heat flux of the above data with $\kappa=100$. (c) The same as (b) but $\kappa=1$. . . . . . . . . . . . . . . . 58

(3.1). Chimney generation when the initial stratification is $T \propto z$, and $S \propto e^{3 z}$. The specified cooling at the top is one unit for the left column, and four units for the right. (a), (d) Temperature. (b), (e) Salinity. (c), (f) Density. . . . . . . 75

(3.2). Information about the salt content for the conditions of figure (3.1). The left column represents a unit heat flux while the right column is four times the cooling. (a), (c) Variations of salinity with depth. (b), (d) Salt content in each density layer weighted by the volume of that layer. In both, the solid line is the situation before the formation of the chimney, the dashed line, after the chimney's generation. 76

(3.3). Same as (3.1) but $T$ and $S$ are $\propto e^{4 z}$. . . . . . . . . . . . . . . 77

(3.4). Same as (3.2) but for the conditions presented in figure (3.3). . . . . . 78

(3.5). Solution to equation (3.2) for the two cases investigated in the text. The solid line corresponds to $T \propto z$ and $S \propto e^{3 z}$, the dashed line to $T$ and $S \propto e^{4 z} . \quad$. $\quad 85$

(3.6). Profiles taken at $33.00^{\circ} \mathrm{N}, 33.50^{\circ} \mathrm{E}$ in the Eastern Mediterranean. (a) Temperature. (b) Salinity. (c) Density. 
(3.7). Reproduction of section 10 of the MEDOC experiment. From Anati and Stommel, (1970).

90

(4.1). The schematic geometric configuration. The interface between the two layers is at depth $h(y)$ and reaches the bottom before the northern wall. Possible characteristics are presented in the lower domain.

96

(4.2). Numerical solutions for the stream function in the lower layer as a function of $a$ and $r$ in equation (4.20). (a) $r=100, a=10,000$. (b) $r=100, a=100$. (c) $r=1$, $a=100$. (d) $r=1, a=2$. (e) $r=1, a=1$. (f) $r=1, a=0.1$. (g) $r=0.01, a=2$. (h) $r=0.01, a=1$. (i) $r=0.01, a=0.1$. (j) $r=0.01, a=0.01$. (k) $r=0.005$, $a=0.1$.

(5.1). The steady state for cosine heat flux and $R_{a}=2 \cdot 10^{4}$. Standard case shown in the left column, density mixing on the right. (a), (d) $T$. (b), (e) $\psi$ (c), (f) $T_{z} .143$

(5.2). The same as (5.1) but for $R_{a}=2 \cdot 10^{5}$.

(5.3). The variations with depth of the terms in the heat equation (5.7b). Line (a), $v T_{y}$; line (b), $w T_{z}$; line (c), $T_{y y}$; line (d), $\kappa T_{z z},(\kappa=1)$. The profiles are taken at four different latitudes with the inserted figure showing the full variation with depth, while the outer one corresponding to the fluid below the thermocline. The Rayleigh number is $2 \cdot 10^{5}$, and the south-north latitude values are normalized between 0 and 1. (a) Section computed at latitude $1 / 16$. (b) Latitude 5/16. (c) Latitude 8/16. (d) Latitude $11 / 16 . \quad$. . . . . . . . . . . . . . . . . . . 149

(5.4). The periodic steady state when seasonal cycle is imposed. The first column is for the situation at the end of summer, while the second is evaluated at the end of winter. This is the standard case with $R_{a}=2 \cdot 10^{4}$. (a), (d) $\psi$. (b), (e) $T$. (c), (f) $T_{z}$. . . . . . . . . . . . . . . . . . . . 154

(5.5). The same as (5.4) but with mixing. . . . . . . . . . . . . 155

(5.6). The same as (5.4) but with $R_{a}=2 \cdot 10^{5}$. . . . . . . . . . . . . . 156

(5.7). The same as (5.5) but with $R_{a}=2 \cdot 10^{5}$. . . . . . . . . . . . . . 157

(5.8). Evolution of $\psi_{\max }$ over a year. Line (a) corresponds to the standard case, (b) to the turbulent mixing case, both with $R_{a}=2 \cdot 10^{4}$. Lines (c) and (d) are the same, but with $R_{a}=2 \cdot 10^{5}$.

(5.9). Quasi steady state for permanent winter condition when $R_{a}=2 \cdot 10^{4}$. The first column represent the fields in the standard case while the second corresponds to the mixing case. (a), (e) $\psi$. (b), (f) $T$. (c), (g) $T_{z}$. (d), (h) $T_{y} . \ldots 161$

(5.10). The same as (5.9) but for $R_{a}=2 \cdot 10^{5}$. . . . . . . . . . . . . . 162

(5.11). Quasi steady state for permanent summer condition. In the first column $R_{a}=2 \cdot 10^{4}$ while in the second $R_{a}=2 \cdot 10^{5}$. The summer state is not affected by the mixing hypothesis. (a), (e) $\psi$. (b), (f) $T$. (c), (g) $T_{z}$. (d), (h) $T_{y}$. . . . 163

(5.12). Evolution of $\psi_{\max }$ while permanent summer or winter conditions are imposed. The solid line corresponds to the standard case, while the dashed to density mixing. The units on the abscissa are real time in years. (a) $R_{a}=2 \cdot 10^{4}$, summer to winter. 
(b) $R_{a}=2 \cdot 10^{4}$, winter to summer. (c) $R_{a}=2 \cdot 10^{5}$, summer to winter. (d) $R_{a}=2 \cdot 10^{5}$, winter to summer. . . . . . . . . . . . . 164

(5.13). The mean fields from the four seasons with $R_{a}=2 \cdot 10^{4}$. Standard case shown in the first column, density mixing in the second. (a), (d) $\psi$. (b), (e) $T$. (c), (f) $T_{z}$. . . . . . . . . . . . . . . . . . 166

(5.14). The same as (5.13) but for $R_{a}=2 \cdot 10^{5}$. . . . . . . . . . . . 167 


\section{Chapter 1}

\section{Preliminaries}

\section{Literature survey}

It has been known for a long time that as one goes deeper and deeper in the ocean, the temperature decreases. Records of this depth dependent temperature profile can be traced at least to 1749 to measurements made off the north west coast of Africa by the captain of the Earl of Halifax, Henry Ellis (1751). In this letter to the Reverend Dr. Hale-who devised the special bucket used for bringing up cold water from the abyssthe slave-trader captain describes, among other things, some of his thoughts and results relating to his deep water temperature measurements. The deepest observation made on that cruise was 5346 feet-which was probably the length of the cable available, and not the true depth. The captain noted that the temperature decreased monotonically to a depth of 3600 feet, and then kept constant up to the maximum depth. The captain being the splendid fellow he was, used the cold water to chill wine, and to combat the hot weather by cold showers.

This phenomena is not altogether self-evident, since if one thinks about a fluid column in equatorial regions, where the air temperature never goes below, say $20^{\circ} \mathrm{C}$ it is only a question of time until all the fluid will reach a temperature of at least $20^{\circ} \mathrm{C}$, even at great depth. Yet, measurements indicate that the waters below are much colder, more like $4^{\circ} \mathrm{C}$. The most common explanation is that these waters come from a colder region, hypothesizing that they were in contact with the atmosphere in a region where 
the air temperature was at least $4^{\circ} \mathrm{C}$; there they sank and contributed to the deep circulation. When measuring the temperature profile in any water column (or for that matter, most other tracers), we actually measure the temperature of fluid particles that came from other places, while only partially succeeding in keeping some of their original properties. Using these properties, we can attempt to trace the particles back to their origin.

Schematically, this circulation pattern is maintained as a balance between downward heat flux (the sea surface is usually the warmest) and upwelling. But the net upwelling should be zero, so presumably there is a narrow region of downward flow where deep water are formed. Observations are not available to directly support the uniform upwelling theory, and indeed Roemmich and Wunsch (1984) showed that over two decades the deep North Atlantic has warmed. However, as the authors pointed out it is hard to tell whether these changes represent long term climatic trends or random fluctuations. Overall, one can probably assume that the ocean is in some equilibrium where, to a good approximation, the excess of heat at equatorial regions is lost to the atmosphere at the poles.

The description above is usually referred to as the thermohaline circulation and is distinguished by a narrow northern branch of sinking cold water, and weak upwelling in the rest of the domain. The word 'asymmetric' appearing in this work is used to stress the difference between the two branches of the flow field, and when used with conjunction with the temperature field, indicates that the temperature structure has a boundary layer character with a thermocline on top of a more isothermal interior.

With the exception of the upper surface, [and the geothermal vents which are not considered as a significant contribution to the global heat budget, but can affect the abyssal flow, Joyce and Speer (1987)] all oceanic boundaries are rigid and insulating. It is through the interaction along the upper interface between the ocean and the atmosphere that the two systems interact, and the ocean is forced. Much of the study in 
the past relied heavily on the mechanical connection between the two systems, where the most notable phenomenon is the stress imparted by the wind to the fluid. After choosing what is believed to be an appropriate small scale mixing and stress parameterization, one can generate Ekman layers, and drive the large scale oceanic circulation.

It is the purpose of this work to touch upon some points relevant to the problem of the thermal forcing, where the mechanical mixing will be included in some of the problems and ignored in others. The circulation induced by the thermal forcing is not only of interest to oceanographers, but to many other disciplines where one forces the system by flux or impressed temperature boundary conditions. In particular, similar problems occur in the atmosphere and the hot magma in the earth's core where the horizontal scale of the forcing is also large as in the ocean.

In the above pictorial description there is a strong emphasis on air-sea interaction as the main mechanism to heat the ocean. This is by and large supported by experimental evidence, and is a consequence of the insulating property of all the other boundaries. Other surface fluxes like evaporation and precipitation are ignored at this stage. A simple procedure for representing the heat loss or gain is to specify the heat flux as a function of position and time. A step further in making the problem more realistic is to allow some degree of feedback by parameterizing the flux as proportional to the difference in temperature between the two systems.

In the usual frame of reference, where $x, y$, and $z$ are the east, north, and upward directions, we will be looking at a problem that is independent of $x$ (by requiring symmetry in that direction) but that still has a meridional velocity field. A configuration like that is referred to in the literature as an axially symmetric system and benefits from a simplified structure and mathematical description compared to the full problem. Because it is common knowledge that meridional boundaries play an important role in the oceanic circulation-for example, by supporting the western boundary layer-one could think that our circulation pattern will have no analogue in the real ocean. But 
this is not the case for the vertical circulation described in this work. The meridional boundaries have less of an effect on the vertical gyre than on the wind driven gyre. We will not be able to duplicate features like deep western boundary currents, but as our results and those summarized here show, the meridional circulation is well defined. The existence of some mean meridional cell is also supported by analysis of observations. Wunsch and Grant (1981) showed that in the North Atlantic the zonally average flow field on a meridional plane is a very stable feature, and even if one employs different assumptions in the inverse method, leading to various circulation patterns, the structure of the vertical gyre is rather similar in all the pictures, with the northward flow deepening towards the Northern North Atlantic, and a deep equatorward flow from high-latitudes.

Some volume of work was devoted in past research to the problem of thermal forcing; albeit much smaller than that directed at the wind forcing. Most researchers simplify the problem by restricting it to the configuration above and investigating a two dimensional annular type circulation. They conclude that even if the impressed temperature field is simple, for example, linear or cosine dependence on latitude, the resultant picture for the velocity and temperature fields can be asymmetric; the temperature having a thermocline occupying a relatively thin layer next to the non-insulating wall, and the velocity having a strong and narrow downward flow in the north, and weak upward flow everywhere else. The asymmetry is a result of the nonlinear interaction between the flow and density fields, and is attributed to the different efficiencies of two important processes in the ocean: the advection of heat in the interior, and the conduction of heat from the surface. These points will be fully treated further on in this work.

In 1950 Stommel presented the first analytic solution for the problem. He carried out to two terms a small parameter series expansion for the temperature and the stream function fields for a non-rotating fluid. The small parameter used was the dimensional 
thermal expansion coefficient $(\alpha)$. Looking at the two zero order equations for the temperature and the stream function, it is apparent that the solution is lacking in many respects. For example, the zero order solution for the temperature indicates that the interior structure is the same as the imposed surface temperature, irrespective of depth. It is the responsibility of the higher order terms to generate the thermocline, and to homogenize the temperature at depth. Clearly this incorrectly opposes the notion that they are acting as a small correction, and indeed, Stommel notes that this is not the case because the series is only slowly convergent. Nonetheless, Stommel's solution is asymmetric for both fields, a non-intuitive result. The reader should note that the symmetry of the stream function in figure (1a) of Stommel's article is opposite to that found in later works. There is broad downwelling in the colder regions, while the upwelling is stronger and confined to the south. The discrepancy with future investigations is real and is not due an error in the figure making, though latter works referring to this paper ignore this difference. In work not included in this thesis it was found that the problem arises because the solution presented by Stommel is outside the range of his small parameter expansion. Namely, that the series solution is correct and does converge, but the small parameter used in the figures is actually rather large $(\approx 204)$, and is outside the radius of convergence.

It is immediately apparent that the analytic solution of even the simplified system used by Stommel (1950) is quite complicated. In an effort to understand the problem, Stommel (1962) devised a system with vertical tubes connected at the top with capillary horizontal tubes, and suspended in a fluid reservoir of constant temperature. The prediction of motion was done by looking at the strongly diffusive and strongly advective limits of the equations. Stommel found that even when one impresses uniform heating and cooling at the top, the fluid goes up everywhere, except at the coldest tube, where it flows down. However, one should note that the asymmetry is partially built into the problem. The flow at the top must be strong and narrow because it is confined 
to capillary tubes, the interior temperature is presumed uniform because the reservoir temperature is fixed, while the flow there must be weak because of its large size. In work not included here, we looked for solutions to the same problem beyond the very diffusive or very advective limits Stommel investigated. For this extended parameter range additional solutions were found - often more than one for the same parameterswhere downwelling was not confined to the coldest pipe, and where several vertical cells could be observed. The mutiplicity of vertical cells might be a realistic feature of the oceanic circulation, [Wunsch and Grant (1981)] but can also suggest that the discretization of the equations to a set of vertical and horizontal pipes is not always successful in describing the fluid behavior.

In the period between these two investigations, Stommel and Veronis (1957) looked at a horizontal layer of fluid which they forced to be gravitationally stable by heating it uniformly from above, and cooling it from below. The mean density structure was approximated by a known constant potential temperature gradient, and, with the exception of vertical advection of this mean field, no advection was present to balance diffusion. The momentum balance is hydrostatic in the vertical, with viscosity balancing horizontal pressure gradients. In the model, the temperature along the lower boundary is slightly perturbed, and the resultant motion and density fields are examined using linear perturbation theory. Cellular asymmetric convective motion develops whose character changes when there is no rotation, uniform rotation ( $f$ plane), or nonuniform rotation ( $\beta$ plane). For atmospheric parameters the developed cell is higher when rotation is imposed, and has a westward tilt when the rotation is made to depend on latitude. The crucial point in this approximation is the known vertical temperature gradient. This leads to a completely linear set-even if rotation is present-which can be solved analytically. The heating and cooling processes also differ conceptually from the oceanic case study, because they both occur at different levels, whereas in the ocean only the upper surface is non-insulating, so the thermal forcing varies on a horizontal 
plane only. By heating at two levels, one can have better control of the stratification and hence the stability of the flow.

The last two presentations gave rise to more experimental and theoretical works which solved the non-rotating problem under different assumptions. In 1967 Sommerville presented a spectral analysis of a nonlinear model where a two dimensional circulation pattern was induced by a horizontal temperature gradient along the bottom of a rectangular tank, with the top fixed at a given temperature. The last choice of a fixed temperature is less suitable to the oceanic case than an insulating wall, and as was mentioned for Stommel and Veronis (1957), this allows changing the lapse rate, which is equivalent to altering the strength of the stratification. The nonlinear terms in the momentum equations were retained, but only vertical viscosity $\left(\nu_{V}\right)$ was present. The solution is derived by writing the temperature as a known solution to the diffusive limit, plus an unknown correction due to advection, followed by expanding both fields in a double Fourier series in both coordinates. Multiplying by the proper trigonometric functions and integrating over the domain leads to a set of prognostic equations for the Fourier coefficients. Sommerville's solution exhibits only a slight asymmetry, due mainly to severe truncation of the Fourier series used in the spectral analysis. As a result of small amount of computer time available, the author allowed only two and three waves to be resolved in the expansion in each direction. Cases where the discrepancies between these two possible truncations were large, were omitted due to insufficient resolution. But even with this truncation the asymmetry in the flow pattern and the thermocline are both clearly visible in the numerical experiments.

The circulation of an axially symmetric system with small aspect ratio (most numerical experiments use an aspect ratio of one or close to it) was also considered by Stone (1968) in a non-rotating and a rotating system. In particular, the scale analysis for very large Rayleigh number was detailed, showing strong vertical and horizontal boundary layers. The limit of a small Rayleigh number was solved analytically like 
Stommel (1950), this time yielding narrow downwelling and broad upwelling. It is important to note that Stone prescribes the heat flux like we will do, and not the temperature. The physical differences between the two will follow shortly.

Other works followed, like that of Beardsley and Festa (1972), who applied a linear temperature along the bottom of an otherwise rigid and insulating rectangular box filled with fluid. The authors used the same dynamical system as Sommerville, with the addition of horizontal diffusion for both the temperature and vorticity equations, and investigated the various parameters associated with the solution of the coupled set. By using a finite difference scheme Beardsley and Festa were able to extend the parameter range of the Rayleigh number (which is a dimensionless measure of the buoyancy) associated with the problem beyond that investigated by Sommerville. They showed that the boundary layer character of the solution becomes more and more evident as the Rayleigh number increases, and presented data supporting a power law relation between the Rayleigh number and the maximum value of the stream function. Even though the largest Rayleigh number they investigated was orders of magnitude smaller than the oceanic values, (see chapter 5) their work is in many respects the last numerical investigation of the general annular circulation problem described at the beginning, and includes a summary of results from previous experimental and theoretical works.

In 1980 Killworth and Manins attempted an analytic solution for the same problem, where quadratic temperature variation was imposed on the bottom, and effort was made to understand the dynamics along the lower boundary. It turned out that the interior temperature is constant, and from turbulence arguments, the interior stream function is proportional to the distance from the intense upwelling northern region. The authors distinguish between two cases: those with a large Rayleigh number where the plume along the warm wall is turbulent; and those with smaller Rayleigh number, where it is laminar and has a constant diffusivity. The linear dependence of the stream function on distance holds for both cases, but in the first case-because there is also 
a term depending on the horizontal distance times the vertical one-the flow has a stagnation point. The solution is sought in terms of similarity solutions, where in order to match to the interior, the stream function and buoyancy have a certain power law dependence on position. The quadratic temperature profile along the bottom wall is actually required by the similarity solution. The coefficients multiplying these solutions are found numerically. The full analysis of the lower boundary layer indicates that two layers are present because the stratification alone cannot satisfy the no slip conditions assumed along the bottom; hence, a layer where nonlinear and viscous terms are dominant must exist. The results are not different from those obtained numerically, but has the obvious benefit of an analytical form, coupled with better understanding of the dynamics, including the relationship between the interior temperature, Prandtl number, and Rayleigh number. The central points in this approach are the utilization of the proper interior forms for the stream function and buoyancy, and the recognition that both the plume and the outflow provide a consistency check in the laminar case and are less important in the turbulent one.

Unlike other works that were set to solve the problem analytically or numerically, Stern (1975, ch. 12.1) uses scaling arguments to derive an expression for the thermocline depth. As was pointed out by Killworth and Manins (1980), the interior temperature used by Stern is the temperature at the coldest spot on the surface. The works cited before suggest that the proper magnitude should be warmer by about $30 \%$ of the of the north south temperature difference, changing the coefficient in his final solution. Simpler arguments leading to the same order of magnitude are also found in Sommerville (1967) and Rossby (1965).

The asymmetric form of the temperature and flow fields can be easily distinguished in laboratory experiments. Among those that initiated interest in the problem is the one done by Rossby (1965). In his experiments, one clearly sees that a linear temperature distribution applied at the bottom of a tank can generate a single asymmetric cell. The 
experimental results indicate that both the cold bottom layer and the upward jet above the warmer side becomes thinner when the viscosity of the fluid is reduced (same as increasing the Rayleigh number). The interior fluid is weakly stratified. The region near the upward jet shows temperature inversion, which is surprising especially because the local Rayleigh number in the experiments was very large; much larger than in any of the numerical realizations. If we are to assume that the region is stable, there must be other dominant effects, or maybe the fluid is not even hydrostatic.

In the works described above the interior circulation is downward, cooled by convection from the upward jet along the warmest wall. The flow warms up by conduction as it advances towards the lower surface, and then goes towards the warmest point in order to rise again. In the case where the fluid is cooled from the top instead of being warmed from below, the physics remains intact. There will be upwelling of cold fluid in the interior, and sinking next to the coldest boundary. The asymmetry-as suggested by Rossby (1965) -is explained as a result of a more efficient heating of fluid by convection than by conduction. These questions, and others relating to the location and extent of the boundary layers will be addressed in chapter 5 .

This brief summary will not be complete without mentioning some of the axially symmetric circulation models done in a meteorological context. These works usually bear little resemblance to the archetypal problem of interest because they deal with a rotational flow on a sphere, and thereby include a more complex dynamics. But in general, much like in the ocean, one develops an asymmetric circulation cell, also known as the Hadley cell, with localized upward motion in equatorial regions.

Schneider and Lindzen (1977) used a linearized numerical model to investigate such flows. Their forcing included a Newtonian cooling law for radiative heating-where the cooling is proportional to the temperature difference from radiative equilibrium-and applied heat sources represent the zonal average large scale cumulus convection. The 
last process is probably the most important driving mechanism in the model. Dissipation of momentum and temperature is done by a vertically varying vertical diffusion coefficient, where no horizontal diffusion is present. In addition, the momentum flux divergence due to cumulus friction is specified, where the mass flux in the hot cumulus is derived from a known heating function. The numerical solutions indicate that cumulus heating and friction drive a Hadley cell that is similar in many aspects to the annual mean observed circulation. In addition, the importance of the sea surface temperature gradient is evident in driving a meridional circulation below $800 \mathrm{mb}$, and in the generation of the ITCZ near the equator. The authors conclude that the model can be successfully used to calculate basic states for stability analysis.

A further study of the same model with the inclusion of nonlinear terms was done by Schneider (1977). In this improved model features like trade winds in the tropics, and surface westerlies to their north are observed.

Another modeling effort was carried out by Held and Hou (1980). There, a nonlinear rotating flow on a sphere is differentially heated. Two crucial assumptions are made: that the viscous forces are weak enough to allow a near conservation of angular momentum; and that the air is statically stable, with a known mean buoyancy that does not change appreciably due to circulation and diffusion. Again, a Hadley cell evolves in the final solution. The nearly inviscid approximations cause some problems, and as Held and Hou admit it is not always clear what form the circulation would take if the viscosity used were smaller than values for which numerical stable solutions were obtained. It seems that the conservation of angular momentum can be applied to the strong boundary flows, and has less success with the interior circulation. We will comment on the importance of friction in the interior in chapter 4. 


\section{Introduction}

Our work here is different in many aspects from previous investigations, although we still keep the same configuration of a fluid confined in a box with rigid and insulating walls, except for the one through which the system is being heated and cooled. We will also comment briefly on the different parameter regimes, the relations between the stream function and the Rayleigh number, and the importance of the Prandtl number when the Rayleigh number varies. Clearly works of this type are far from being exhausted, and deserve much more attention, especially because the oceanic study corresponds to a vary large Rayleigh number. For example, the numerical experiments did not go much beyond a Rayleigh number of order $10^{5}$, where laboratory measurements are available with a parameter five orders of magnitude larger. This highly unstable region is not well understood; hence often one employs other arguments like radiation convection equilibrium used in the atmosphere.

The most notable aspects of this work are three-fold. First we present a mixing parameterization to which we will refer as "convective overturning". A variation of this parameterization is used in general circulation models like Bryan an Cox (1968), but was not employed in the works summarized before for the meridional circulation. One of the benefits of this approach is that the final density field is everywhere stable or marginally stable. Previous works could only reach a stably stratified solution over the whole domain by a priori imposing a mean temperature field, [Stommel and Veronis (1957), Held and Hou (1980)]. Even Rossby's (1965) laboratory experiments with Rayleigh numbers of order $10^{7}$ to $10^{10}$ show temperature inversions along the bottom boundary. In cases where the main stratification was not imposed a priori, large unstable or weakly unstable regions exist as part of the steady state solution, whereas in our work, an overturning process will lead to marginal stability in those regions.

Secondly, we investigate the effects of the time dependent forcing on the stratification and flow fields. It will turn out that dramatic seasonal changes can be observed. 
In particular, distinct differences are measured when driving the circulation by mean forcing compared to averaging seasonal states. These will be analyzed in chapters 2 and 5. The effects of a seasonal cycle is investigated in other numerical models, in particular, primitive equations general circulation models applied to tropical regions like Philander and Pacanowski (1984). The question of variability was not addressed in the past in the configuration presented in this work, and more importantly, the difference between the two averaging schemes presented above is seldom touched upon. The work by Bryan and Lewis (1979) suggest that the two procedures will yield different results as observed in chapter 2 . Generally, because most fields like heating function, wind, and sea surface elevation, show an annual variability, it is difficult to distinguish between cause and effect. In the simplified context of this work, where all forcing but the heating are neglected, it will be easier to note the effects of a seasonal cycle in the thermal forcing.

Thirdly, with the exception of Stone (1968), our boundary conditions are different from other works. The heating function along the upper boundary is specified in terms of flux and not a given temperature, and thus is a direct statement of the forcing. When the temperature is specified, the heat flux-which is the real forcing in the problem-is determined as part of the solution, and might not be always realistic. For example, Stone (1968) suggests that the strong asymmetry found by Stommel (1962) is attributed to the fact that Stommel's applied thermal conditions presumably correspond to an asymmetric flux with cooling above the coldest pipe only. By specifying the flux and not the temperature we avoid such problems, and can investigate the asymmetric circulation resulting from a specified symmetric forcing. Other differences between specifying the flux and the temperature are discussed in chapter 5 , where the relation between heat flux and the oceanic horizontal surface temperature gradient is analyzed.

Unlike most other works, we neglect nonlinear terms a priori except in the heat equation. The role played by the nonlinear terms is important in the boundary layer 
analysis when one requires no slip conditions along the non-insulating boundary. Killworth and Manins (1980) indicated that they are dominant in the upper boundary layer of the highly asymmetric solutions, drawing energy from the mean flow. Still, for free slip conditions, Stommel's (1962) treatment hints that maybe the nonlinearity in the density equation is enough, since his pipe solution is highly asymmetric, but his 'momentum' relation is linear. Also, Beardsley and Festa (1972) conclude that the advection of vorticity by the flow field is only about $5 \%$ of the other terms for their numerical experiments with a Rayleigh number of order $10^{5}$.

As a last point, in order to resemble the oceanic case, we heat and cool the annulus from the top, and not from from below. The location of the non-insulating boundary is more a question of convenience, because we can always interchange cooling at the top with heating along the bottom.

In chapter 2 the density field of a fluid in an annular configuration that is heated from the top is examined. The fluid is diffusive and viscous enough so that no flow field is present (at least to the lowest order). The diffusion coefficient is a variable that depends on the local stratification. A variety of heat fluxes are used as boundary conditions, including one based on data collected by Oort and Vonder Haar (1976). The results show that the fluid is forced to sink in all regions where the ocean is being cooled by the atmosphere. The vertical extent of these sinking regions is a strong function of horizontal position. Different temperature structures from various averaging procedures are analyzed.

The third chapter uses the parameterization developed in the second in a situation where a local buoyancy loss causes strong vertical mixing. A simple model, based on mixing only without motion, is developed and used to show that the effects of vertical mixing are important in the formation process of water masses like mode water found in the world's oceans. Examples from the Mediterranean Sea are treated. 
The fourth chapter presents an analytical model for the vertical circulation with the dynamics described by a mixed layer lying on top of an interior water mass. The model is fairly general and can be realized at locations like the subpolar gyre where two conditions should be met; a wall at the poleward side, and a sense of the circulation where waters are being pumped from the mixed layer into the interior. The model helps in establishing several features that are unique to the axially symmetric problem: in particular, the important role of the weak dissipation of angular momentum; and the surprising fact that the passage of information in the system is against the flow field.

The model provides a general dynamical framework for the circulation at northern regions, and can be complemented by other local processes of deep water formation. The formation events are believed to happen in a very limited number of small sinking regions over the world oceans. It is thought that deep waters are formed in two different distinct processes: open water convection where the water is pumped down in chimneys where properties are homogeneous in the vertical, and sinking along continental shelves. The first process is documented in the Mediterranean [MEDOC Group (1970)], the Weddell polynya [Gordon (1978)], and to lesser extent in other regions like the Norwegian Sea [Carmack and Aagaard (1973)] and the Labrador Sea [Clarke and Gascard (1983), Lazier (1973)]. Suggested models can be found in Killworth (1976), Killworth (1979), and Martinson et al. (1981). Note, that the last two models that deal with the formation process in Antarctic regions have salt rejection due to a freezing ice cap, a process that helps produce warm and salty 'heavy' water above cold but fresh 'light' ones. The formation near continental shelves is also well observed, especially in the Weddell Sea [Gill (1973), Foster and Carmack (1976)] the Adélie coast [Gordon and Tchernia (1972)], and the Ross Sea [Jacobs et al. (1970)]. A review of this process is found in a paper by Killworth (1983), where most of the observations are recorded and referenced. The general dynamics described in chapter 4 is not detailed enough to 
describe formation processes where things like sea ice formation and salt rejection are needed, but on the other hand is appropriate in much broader regions where there is buoyancy loss at the surface.

Chapter 5 returns to the archetypal problem that was surveyed in the previous section, namely, that of the axially symmetric fluid differentially heated from the top. As mentioned before, we extend previous works in several directions, of which the two most important ones are the introduction of strong vertical density mixing when the stratification is unstable, and analysis of the time dependent problem. 


\section{Governing Equations}

The task of setting up a mathematical formulation to describe a physical problem can be quite complicated, where several issues need be addressed. Among those questions are: the proper dynamics, in particular the momentum equations; the thermodynamics, and its interaction with the dynamic variables; and formulation of boundary conditions. The last can include topography, wind stress imparting momentum to the system, heat fluxes, and evaporation and precipitation which not only act to change the salinity, but are sometimes treated as a mass source-sink.

Numerical simulations, which are often required to solve the resultant set of equations, introduce other complications, in particular, the resolution problem. As a result, we are often forced to make some type of a closure statement in order to be able to cut off the high wave numbers, while still accounting in some fashion for scales not resolved in the model.

The general dynamical framework which is often used to describe general circulation problems is usually written as:

$$
\begin{aligned}
u u_{x}+v u_{y}+w u_{z}-f v & =-\frac{1}{\rho} p_{x}+\nu_{H}\left(u_{x x}+u_{y y}\right)+\nu_{v} u_{z z} \\
u v_{x}+v v_{y}+w v_{z}+f u & =-\frac{1}{\rho} p_{y}+\nu_{H}\left(v_{x x}+v_{y y}\right)+\nu_{v} v_{z z} \\
u w_{x}+v w_{y}+w w_{z} & =-\frac{1}{\rho} p_{z}-g+\nu_{H}\left(w_{x x}+w_{y y}\right)+\nu_{v} w_{z z} \\
u_{x}+v_{y}+w_{z} & =0 \\
u \rho_{x}+v \rho_{y}+w \rho_{z} & =\kappa_{H}\left(\rho_{x x}+\rho_{y y}\right)+\kappa_{v} \rho_{z z} \\
\rho & =\rho_{0}\left[1-\alpha\left(T-T_{0}\right)\right]
\end{aligned}
$$

where we used the conventional notations with $x, y$, and $z$ as the east, north, and upward directions, and $u, v$, and $w$ for the velocity components in these directions. $\rho$ and $T$ stand for the density and temperature, $\rho_{0}$ and $T_{0}$ denote the reference density and temperature, $\alpha$ indicates the thermal expansion coefficient, $p$ the pressure, $g$ the 
gravitational acceleration, and $f$ the Coriolis parameter. $\nu_{z}$ and $\nu_{V}$ denote the horizontal and vertical components of the kinematic viscosity, and $\kappa_{H}$ and $\kappa_{V}$ represent the horizontal and vertical components of the thermal diffusivity. The viscosity and diffusivity in (1.1) are assumed to be constants. Later in this work we will relax this assumption. The formal effect in the representation of the equations will be that terms like $\nu_{z} u_{x x}$ will be replaced by $\left(\nu_{H} u_{x}\right)_{x}$.

For simplicity, our fluid is confined in an annulus with its north and south boundaries paralleling latitude lines. As was explained before, in this geometric representation we restrict ourselves to problems that do not exhibit $x$ dependence. This makes it easy to use the continuity equation (1.1d), and define a stream function

$$
\begin{aligned}
& \psi_{z}=-v \\
& \psi_{y}=w
\end{aligned}
$$

Our set of six equations for the unknowns $u, v, w, \rho, T$, and $p$, can now be reduced to three: the $x$ momentum equation, the vorticity equation, and the heat equation.

$$
\begin{aligned}
J(\psi, u)+f \psi_{z} & =\nu_{y} u_{y y}+\nu_{v} u_{z z} \\
J\left(\psi, \nabla^{2} \psi\right)-f u_{z} & =\alpha g T_{y}+\nu_{H} \nabla^{2} \psi_{y y}+\nu_{v} \nabla^{2} \psi_{z z} \\
J(\psi, T) & =\kappa_{z} T_{y y}+\kappa_{V} T_{z z}
\end{aligned}
$$

where $J(a, b)=\frac{\partial a}{\partial y} \frac{\partial b}{\partial z}-\frac{\partial a}{\partial z} \frac{\partial b}{\partial y}$ is the Jacobian operator, and we have used the Boussinesq approximation to neglect the variations in density except where coupled to gravity. The boundary conditions required to solve this set involves knowing the temperature or heat flux around the domain, as well as information on the velocities $\left(\psi, u\right.$, and $\left.\nabla^{2} \psi\right)$.

Exploring the interaction between different scales in the above set is often more transparent if we use the equivalent dimensionless equations. Using asterisks to denote 
dimensionless quantities, we introduce the following scales

$$
\begin{aligned}
y & =L y^{*} \\
z & =D z^{*} \\
\psi & =\phi \psi^{*} \\
f & =f_{0}\left(1+\frac{\beta L}{f_{0}} y^{*}\right)=f_{0}\left(1+\beta^{*} y^{*}\right)=f_{0} f^{*} \\
u & =U_{0} u^{*} \\
T & =T_{0} T^{*}
\end{aligned}
$$

and redefine our Laplace operator to be $\nabla^{2}=\left(\frac{D^{2}}{L^{2}} \frac{\partial^{2}}{\partial y^{2}}+\frac{\partial^{2}}{\partial z^{2}}\right)$. Note, that the last two scales, $U_{0}$ and $T_{0}$, are external parameters. The relation between $U_{0}$, and the forcing parameter $T_{0}$ will be established shortly. It is also helpful to introduce some dimensionless parameters

$$
\begin{aligned}
F & =\frac{f_{0}^{2} L^{2}}{g D}, \\
\epsilon & =\frac{U_{0}}{f_{0} L}, \\
\kappa & =\frac{\kappa_{v} L^{2}}{\kappa_{H} D^{2}}, \\
\nu & =\frac{\nu_{v} L^{2}}{\nu_{B} D^{2}}, \\
E & =\frac{\nu_{v}}{f_{0} D^{2}}, \\
\sigma & =\frac{\nu_{v}}{\kappa_{v}}, \\
\delta & =\frac{\phi D}{\nu_{v} L} .
\end{aligned}
$$

As we will show momentarily, not all of these seven parameters are independent, and it will be convenient to relate $\epsilon$ and $\delta$ to the other quantities. Employing these definitions 
gives the following dimensionless set

$$
\begin{aligned}
\delta J(\psi, u)+\frac{\delta}{\epsilon} f \psi_{z} & =\left(\frac{1}{\nu} \frac{\partial^{2}}{\partial y^{2}}+\frac{\partial^{2}}{\partial z^{2}}\right) u \\
\delta E^{2} J\left(\psi, \nabla^{2} \psi\right)-\frac{\epsilon}{\delta} f u_{z} & =\frac{\alpha T_{0}}{\delta F} T_{y}+E^{2}\left(\frac{1}{\nu} \frac{\partial^{2}}{\partial y^{2}}+\frac{\partial^{2}}{\partial z^{2}}\right) \nabla^{2} \psi \\
\delta \sigma J(\psi, T) & =\left(\frac{1}{\kappa} \frac{\partial^{2}}{\partial y^{2}}+\frac{\partial^{2}}{\partial z^{2}}\right) T
\end{aligned}
$$

Now $\psi, u, T, f, y$ and $z$ are $O(1)$, and we dropped the asterisks for neatness.

Further simplification of (1.6) is often possible by relating some of the scales used in deriving the dimensionless set. One of the more popular assumptions leading to a thermal wind balance in the vorticity equation (1.6b) while balancing the $v$ velocity component by $u_{z z}$ (remember that the system is axially symmetric) is derived by assuming that $\delta=\epsilon$, and that $\frac{\delta}{\epsilon}=\frac{\alpha T_{0}}{\delta F}$. In this way, the external parameters are now related $U_{0}=\alpha T_{0} \frac{g D}{F L}$, as well as $U_{0}=\phi \frac{f_{0} D}{\nu_{v}}$, and $\delta=\epsilon=\frac{\alpha T_{0}}{F}$. These assumptions yield the familiar set written in terms of the Rossby $(\epsilon)$, Ekman $(E)$, and Prandtl $(\sigma)$ numbers

$$
\begin{aligned}
\epsilon J(\psi, u)+f \psi_{z} & =\left(\frac{1}{\nu} \frac{\partial^{2}}{\partial y^{2}}+\frac{\partial^{2}}{\partial z^{2}}\right) u \\
\epsilon J\left(\psi, \nabla^{2} \psi\right)-f u_{z} & =T_{y}+E^{2}\left(\frac{1}{\nu} \frac{\partial^{2}}{\partial y^{2}}+\frac{\partial^{2}}{\partial z^{2}}\right) \nabla^{2} \psi \\
\epsilon \sigma J(\psi, T) & =\left(\frac{1}{\kappa} \frac{\partial^{2}}{\partial y^{2}}+\frac{\partial^{2}}{\partial z^{2}}\right) T .
\end{aligned}
$$

For some of the approaches in the chapters 4 and 5 it is convenient to have a different scale for the stream function than the one implied above. In those cases it is more transparent to use the set (1.6) than the set (1.7). In any case, the appropriate assumptions will be clearly stated at the beginning of each chapter.

When the Rossby $(\epsilon)$ and Ekman $(E)$ numbers are small, and we further justify neglecting the nonlinear advection of vorticity by $\epsilon<E^{2}$, the $u$ dependence can be eliminated and $(1.7 \mathrm{a}-\mathrm{b})$ can be reduced to one equation relating $T$ and $\psi$ : [The absence of inertial terms is also of advantage numerically. Even though we are looking for a 
steady state where no propagating waves exist, the solution is the end result of a time dependent spin up problem. If there are inertial terms, the time step is short, and is governed by the fast gravity waves (and also Rossby waves if there is no east-west symmetry). When the fast waves are suppressed, time stepping is a faster process.]

$$
\psi_{4 z}+\frac{f^{2}}{E^{2}} \psi=-\frac{1}{E^{2}} T_{y}
$$

where we retained the vertical diffusion terms relative to the horizontal one. The high order derivative with respect to $z$ comes from the $z$ momentum equation. This term is usually small because to first order our flow is hydrostatic. Nonetheless, it should be retained if we want to solve for the Ekman layers at the top and bottom. The heat equation (1.7c) remains the same as before, and the leading order terms depend on the relative size of the Prandtl $(\sigma)$ and Rossby $(\epsilon)$ numbers. Equations (1.8) and (1.7c) can be solved for proper formulation of boundary conditions. For instance, assume a temperature structure, use it to compute the stream function from (1.8)-which has an analytic solution-and apply the result for the stream function to improve the temperature field, and so on.

The zonal velocity is determined by integrating the $x$ momentum equation (1.7a), which for our approximation is simply $f \psi_{z}=u_{z z}$. The result is

$$
u=f \int_{0}^{z} \psi d z+\tau^{\text {wind }} z \text {. }
$$

The wind stress enters the result by our assumption that we can parameterize the velocity shear at the top as a stress, thus, $u_{z}(y, z=0)=\tau^{\text {wind }}(y)$. Note, that the kinematic viscosity need not be constant here. This solution was made possible because we kept the high order derivative term in (1.8). Without this term one cannot require $u_{z}=\tau^{\text {wind }}$.

Clearly, the last two equations are only an example from the wealth of possible dynamics hidden in the set (1.6). In what follows we will focus on specific processes and 
will justify picking up parts of these equations, employing different balance schemes. The aim of these manipulations is to simplify the description of the complicated structure of the fluid behavior. It is important, even in cases where one can write the mathematical formulation properly, to identify the terms dominating the fluid behavior from those of minor contribution, or those that have an effect only in limited regions. 


\section{Chapter 2}

\section{The effects of heating in the absence of a flow field}

\section{Introduction}

We start our examination of the thermal forcing by looking at a case with no flow field, where only mixing is relevant. In relation to the governing equations (1.6), this approximation is the same as assuming that $\delta$ is small enough (or that $E>\epsilon$ ) so that for a given Prandtl number $\sigma, \delta \sigma \ll 1$. The small correction to the temperature field induced by the weak velocity field can be computed by using the vorticity equation (which is basically the thermal wind relation when $E^{2} \ll 1$ ) to get the meridional velocity component, and the $x$ momentum equation to get the stream function. Mathematically, the zero order problem boils down to solving the diffusion equation in a rectangular box for specified boundary conditions. The analytic solution to this textbook problem is readily available. It is the physical interpretation of the results that complicates the problem and makes it interesting for the oceanic case.

While solving the diffusion equation for the temperature, several different surface boundary conditions will be analyzed; including those where the flux is specified, when it is time dependent, and when the air temperature is given. We find that in regions where the fluid is cooled from above, the vertical stratification becomes unstable. This leads us to introduce the concept of convective overturning. The idea behind it is very simple. In places where heat is taken away from the upper portion of the fluid, there may exist a layer of heavy fluid on top of lighter one. In these locations we make the 
vertical mixing more vigorous than in other places, thereby letting the vertical diffusion rapidly smooth the undesired density gradients. This concept will be related to the parameterization of turbulent and mesoscale mixing and will be widely used throughout chapters 3 and 5 .

\section{Heat equation}

The heat equation $(1.6 \mathrm{c})$ relates the stream function to the temperature. In order to examine of the importance of thermal forcing, we eliminate the stream function dependence by looking at the diffusive limit of this equation. Apart from simplifying the problem, elliptic equations illuminate the importance of boundary conditions because the solution in any interior point is some average of its surrounding. Our equation is written as

$$
T_{y y}+\left(\kappa T_{z}\right)_{z}=0
$$

where as before $\kappa=\frac{\kappa_{V} L^{2}}{\kappa_{B} D^{2}}$, and is treated as a constant, and we assumed that the horizontal diffusion coefficient is independent of position. Note, however, that by grouping the $\kappa T_{z}$ inside the brackets, we leave open the possibility that in the future $\kappa$ will depend on position. For no fluxes through the walls, $T_{y}=0$ at $y=0$ and $y=1$, $T_{z}=0$ at $z=0$, and specified surface heat flux $\kappa T_{z}=Q$ at $z=1$, the solution to this diffusive limit is

$$
T=A_{0} \cos (\pi y) \cosh \left(\frac{\pi z}{\sqrt{\kappa}}\right)
$$

with

$$
Q=A_{0} \sqrt{\kappa} \pi \sinh \left(\frac{\pi}{\sqrt{\kappa}}\right) \cos (\pi y)
$$

where $A_{0}$ is constant. For cases where the heat flux $Q$ does not exhibit a cosine behavior in $y$, we can simply write it-and the solution-as a Fourier sum.

Our simple diffusion equation actually contains some interesting information. Take typical scales of $L=4000 \mathrm{~km}, D=4 \mathrm{~km}, \kappa_{v}=1 \mathrm{~cm}^{2} / \mathrm{sec}$ and $\kappa_{z}=10^{6} \mathrm{~cm}^{2} / \mathrm{sec}$, and 
get $\kappa=1$. Now the temperature structure exhibits a strong vertical dependence, where the difference between the top and bottom temperatures can be large $[\cosh (\pi)-1] \approx$ $10.6^{\circ} \mathrm{C} \quad\left(A_{0}=1\right)$. This result is not an obvious one, because the fluid is confined to a very thin slab (aspect ratio of 1000 ), and one would not immediately expect that applying an order one temperature difference along the top of this slab, will translate to order one changes in the vertical. The reason behind the phenomena is of course the large difference between the horizontal and vertical mixing coefficients, preferring horizontal processes over vertical ones. Observations also suggest that in the ocean, vertical temperature changes are large. For example, Wüst (1935, pp. 3), and Iselin (1939) noticed that a $T-S$ diagram of surface water would correspond closely to a $T-S$ relation from a hydrographic station, implying that the surface structure closely resembles the vertical one. These observations are usually considered as evidence that advection and diffusion processes are more vigorous along density surfaces than across them.

It is interesting to see what happens if we follow this idea, and replace the horizontal and vertical diffusivities by along and across-isopycnal mixing coefficients. This amounts to relating the mixing coefficient $\kappa$ 's in the cartesian system to the along and across-isopycnal coefficients by a second order tensor whose arguments are the projections of the vertical and horizontal diffusion coefficients along and across isopycnal surfaces [Redi (1982)]. The components of the new isopycnal mixing coefficients are now $\kappa^{t}=\left(A_{a l}, A_{a l}, A_{a c}\right)$ as opposed to the rectangular coordinate representation $\kappa^{R}=\left(\kappa_{H}, \kappa_{H}, \kappa_{v}\right)$. The across and along-isopycnal diffusion coefficients are denoted by $A_{a c}$ and $A_{a l}$. The tensor can be written as

$$
\kappa_{i j}=A_{a l}\left[\delta_{i j}+\left(\frac{A_{a c}}{A_{a l}}-1\right) \frac{\partial_{i} \rho \partial_{j} \rho}{\rho_{x}^{2}+\rho_{y}^{2}+\rho_{z}^{2}}\right]
$$

where $i$ and $j$ vary over the three coordinates, and $\delta_{i j}$ is the unit tensor whose elements are one when $i=j$, and zero otherwise. 
Assuming that the equation of state (1.1f) has linear dependence on temperature only, there are no gradients along density surfaces because there are no contributions to the density field from either salinity or pressure. For this case, the dimensional heat equation $\partial_{i}\left(\kappa_{i j} \partial_{j} T\right)$ collapses immediately to $\nabla\left(A_{a c} \nabla T\right)=0$. If $A_{a c}$ is constant, the equation is completely equivalent to $\kappa_{z} T_{y y}+\kappa_{v} T_{z z}=0$ where we require $\kappa_{B}=$ $A_{a c}=\kappa_{v}$. This is of course the isotropic limit, which corresponds to a very large value of $\kappa=L^{2} / D^{2}$ in our scaled equation (2.1). Because the vertical mixing coefficient is large enough, the horizontal mixing coefficient small enough, and the slab of fluid so thin, the temperature structure in this case is depth independent, so that the latitudinal variations of the temperature impressed at the top of the basin persist all the way to the bottom. This result shows that in our problem the treatment in density coordinates is equivalent to isotropic mixing coefficients in regular space. It is independent of possible technical difficulties of implementing surface boundary conditions in density coordinates, and holds true for a three dimensional system as well. It suggests that in our problem it is not proper to use along and across-isopycnal mixing, and points out the importance of a realistic equation of state when dealing with isopycnal mixing, (in particular, the nonlinearities in the density field) and the role played by the circulation that advects this temperature field.

Another possible model for the role of mixing was postulated by Armi (1978). The author hypothesized that it is possible to explain the stratification in the deep water as a combined effect of vertical mixing at boundaries and topographic features, followed by lateral advection and diffusion along isopycnal surfaces. According to this argument, the stratification is determined by mixing along side walls and to a much lesser extent by the anisotropic nature of the fluid. In any case, we would continue to employ the anisotropic model because it gives a crude parameterization of the circulation.

The effects of the circulation is examined in chapter 5 where a similar problem is treated while including the flow field. One of the limits investigated (although not 
presented in this report) is the absence of horizontal diffusion in the axially symmetric configuration. This is an extreme case where $\kappa$ is infinitely large, whereas in the isotropic example before, it was the square of the inverse aspect ratio-about $10^{6}$. We find that even under these extreme conditions, there is a clear thermocline structure, indicating that the flow field is important in determining the final shape of the isopycnals.

\section{Convective overturning}

The solution for $T$ in (2.2) has unstable stratification over half the domain; namely, $T_{z}<0$ for $y \in\left(\frac{1}{2}, 1\right)$. This of course is not physically plausible, and one would tend to assume that wherever a situation is reached where heavy water lies on top of lighter ones, the fluid will rapidly mix to form a marginally stable water column. In order to overcome the possible physical instability of the solution (2.2), the diffusive limit was solved numerically with a vertical diffusion coefficient that depends on $T_{z}$,

$$
T_{y y}+\left(\kappa T_{z}\right)_{z}=0 \quad \text { with } \quad \kappa= \begin{cases}O(1), & T_{z} \geq 0 \\ \rightarrow \infty, & T_{z}<0\end{cases}
$$

The transition from (2.1) to (2.4) introduces a new nonlinear problem associated with

our previous linear description. In northern regions, the above parameterization of fast vertical mixing causes the water column to have an almost constant temperature profile. This parameterization is similar to the one used by Bryan and Cox (1968) [equation (3.20a) in their article], but its execution is different. Bryan and Cox takes care of the infinite mixing by a procedure that is equivalent to replacing the unstable stratification in a density column by some arithmetic mean of the density in that column. As opposed to that, our mixing process involves increasing the coefficient to a large but finite value. Following Bryan and Cox, the stratification should always be relaxed at locations where the fluid is cooled (because there the surface stratification is not stable, thus, at every time step a new mean density profile is calculated), hence 
a real steady state is not achieved. In our scheme a true steady state can be reached, and the numerical implementation is rather easy. In particular, it is easier to use our approach in cases where the Prandtl number is fixed, where we also mix momentum as well as density. Overall, the differences between the two schemes are small in both the technical and physical aspects, and numerical simulations should lead to similar results using either way.

For an imposed surface heat flux of $\cos (\pi y)$, the schematic result is represented in figure (2.2) b, and is analyzed later on. It is clear that the temperature structure is now different from the solution (2.2) which is represented in figure (2.2)a. The heavy line in figure (2.2)b marks the interface $\eta(z)$ between the regions where $T_{z} \geq 0$ (south) and $T_{z}<0$ (north).

Equation (2.4) is not only different mathematically from the linear problem (2.1), but has different underlying physics. The diffusion coefficients $\kappa_{H}$ and $\kappa_{V}$ are representative of mesoscale mixing parameterization, indicating that mesoscale features, like eddies, act to mix the ocean horizontally much more than vertically. But this is not the only scale on which mixing acts. There is also small scale turbulent mixing, which is a process found in the ocean but not resolved in our parameterization. In equation (2.4) we deliberately let the mixing coefficient depend on the stratification in order to include this turbulent process. Overall, in the stably stratified regions the mesoscale mixing controls the fluid behavior, whereas in unstable regions, it is the turbulent processes that determine the temperature structure.

The numerical simulations presented later on in this chapter show that there are two regions corresponding to the different values of $\kappa$ in equation (2.4). In the southern region the diffusivity is $\kappa_{1}=O(1)$, whereas in the northern region it is much larger, $\kappa_{2} \gg 1$. Technically, if the interface between the two regions is specified, one can solve (2.1) in each region, and determine the temperature everywhere. Because of the new shape of each domain, the solution will be more complicated than (2.2). But 
determining the shape of the interface is part of our solution. In the following paragraph we present a simple approach to investigate the temperature structure in the large $\kappa$ region. Although we will not be able to determine the shape of the interface, this solution shows the approximate temperature structure in the unstable region, and will also suggest that possibly the shape of the interface is independent of the ratio $\kappa=\kappa_{2} / \kappa_{1}$. This result was also verified by numerical simulations, that in addition demonstrated the dependence of the shape on $\kappa_{1}$.

In the north, $\kappa_{2} \gg 1$ and can be used in a small parameter expansion $\left(\kappa_{2}^{-1}\right)$. The equation can be written as $T_{z z}+\kappa_{2}^{-1} T_{y y}=0$, where the flux at the top $(z=1)$ is specified $\left.\kappa_{2} T_{z}\right|_{z=1}=Q$. Expanding the temperature in a power series of the small parameter $\kappa_{2}^{-1}$,

$$
T=T^{(0)}+\kappa_{2}^{-1} T^{(1)}+\cdots+\kappa_{2}^{-n} T^{(n)}+\cdots
$$

shows that the zero order equation is $T_{z z}^{(0)}=0$, so that $T_{z}^{(0)}=f(y)$. In addition, the expansion of $T$ for the top boundary condition gives

$$
\kappa_{2} T_{z}^{(0)}+T_{z}^{(1)}+\kappa_{2}^{-1} T_{z}^{(2)}+\cdots+\kappa_{2}^{1-n} T_{z}^{(n)}+\cdots=Q=O(1) .
$$

Because the surface heat flux is order one, we must also assume that $T_{z}^{(0)}=0$, so

$$
T^{(0)}=g(y)
$$

The function $g(y)$ is determined from the first order set

$$
T_{z z}^{(1)}+T_{y y}^{(0)}=0
$$

The matching condition at the top leads to $T_{z}^{(1)}=Q+g_{y y}(1-z)$, and that at the bottom (which can be the interface) to $g_{y y}=\frac{Q}{d-1}$ with $0 \leq d(y) \leq 1$ as the depth on the interface. Thus, we know that

$$
\begin{aligned}
& T^{(0)}=\int d y \int d y \frac{Q}{d-1} \\
& T^{(1)}=\frac{Q}{2(1-d)} z^{2}+a(y) .
\end{aligned}
$$


As before, we have to go an order further to determine $a(y)$. The most important result of this expansion is that the zero order solution is independent of depth and is controlled by the forcing only. One should note, that when $d=0$, summing the terms in the series expansion results in a $\cos (\pi y) \cosh \left(\pi z / \sqrt{\kappa_{2}}\right)$ where $Q=\cos (\pi y)$. This result is of course due to the fact that the analytic solution (2.2) is valid for any $\kappa_{2}$. For large diffusivities, $\cosh \left(\pi z / \sqrt{\kappa_{2}}\right)$ is approximately one and so $\kappa_{2}$ does not enter the zero order solution. This important observation lies behind our success in solving for the temperature in the northern region without knowing the shape of the domain itself (remember that the form of the interface is yet not determined). So we have shown that to a good approximation, neither the temperature, nor the shape of the interface depends on $\kappa_{2}$. We will see shortly that it does depend on the diffusivity in the southern region $\kappa_{1}$.

The solution above is not sufficient to determine the curve representing the interface in figure (2.1), but matching the solutions on its two sides require continuity of temperature and flux across the interface.

$$
\begin{aligned}
T^{(\mathrm{i})} & =T^{(\mathrm{ii})} \\
\cos \theta\left(T_{y}^{(\mathrm{i})}-T_{y}^{(\mathrm{ii})}\right) & =\sin \theta\left(\kappa_{2} T_{z}^{(\mathrm{ii})}-\kappa_{1} T_{z}^{(\mathrm{i})}\right)
\end{aligned}
$$

where the interface is inclined at an angle $\theta(z)$ with respect to a vertical line, and $\eta(z)$ is the displacement of the interface from a reference vertical line-say the line intersecting the surface where the specified heat flux $\kappa T_{z}=0$, so $\eta(1)=0$. Results like those presented in figures (2.2) indicate that $\eta(z)$ is very large and a perturbation expansion around the reference line is not always a good idea.

The continuity of flux (2.5b) can be simplified further by noting that $T_{z}=0$ on the interface. The fact that the vertical temperature gradients are positive to the south of it and negative north of it is not a rigorous justification, but the figures in chapter 5 , where $T_{z}$ contours are presented, lend additional support to the idea. (2.5b) 


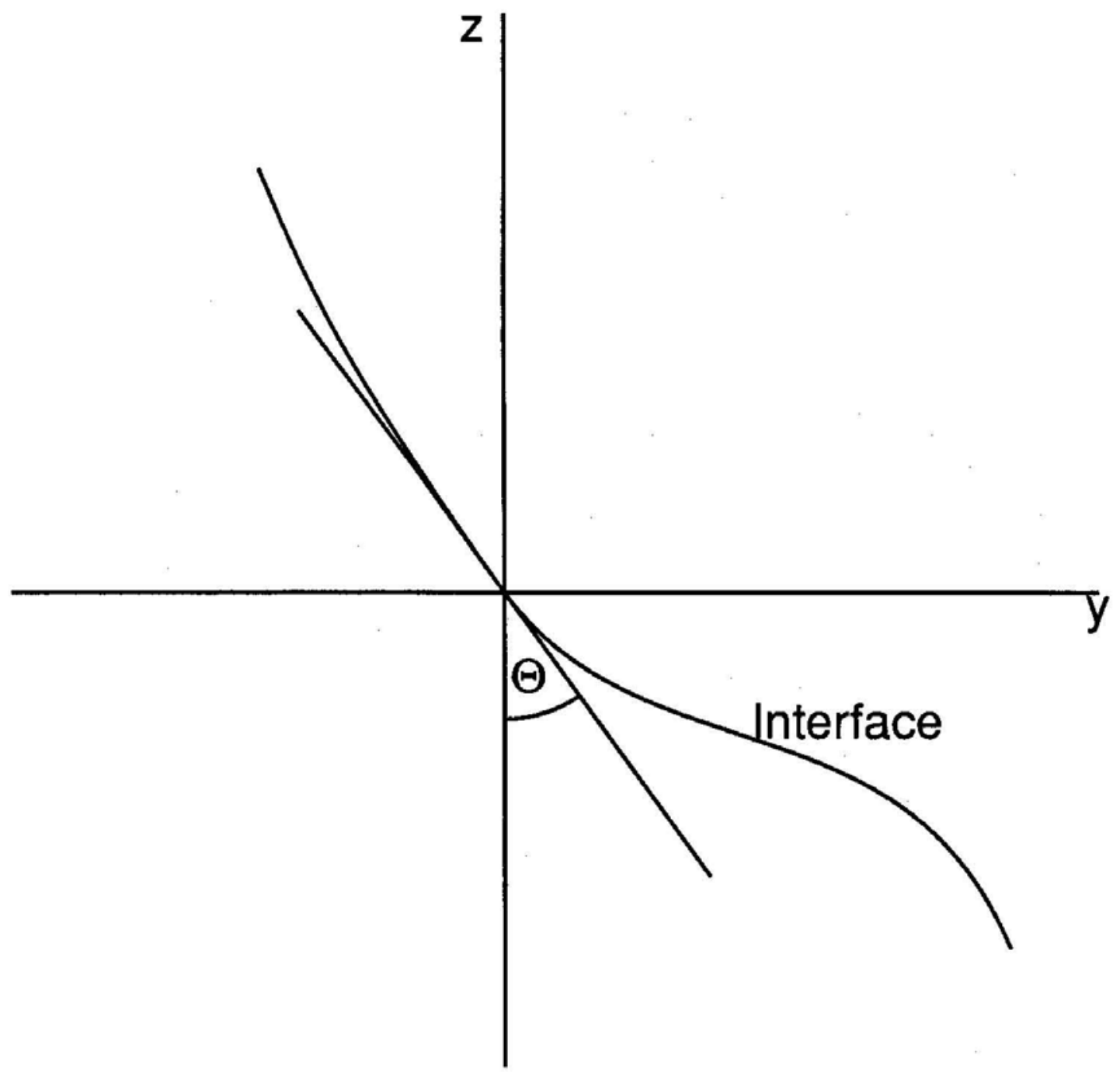

Figure (2.1). A sketch of the interface between the convective overturning region and the stable one. 
would now suggest that $T_{y}$ is also continuous across the interface. In addition to the continuity of temperature, one should also remember that the temperature in the north can be determined everywhere to within a constant from the specified forcing $Q$, thus, in particular, we know the temperature on the interface.

The problem now remains to solve (2.1) in the southern region under the specified boundary conditions of a given heat flux at the top (it is actually heating only because we already solved for the region that is cooled) and no flux through the southern and bottom walls. The shape of the northern extent of the domain is still unknown, but is replaced by two boundary conditions: the specified temperature, and $T_{z}=0$. The fact that the net flux through the interface to the northern region equals the input of heat at the top is automatically satisfied by any steady state solution of the diffusion equation. Solving the elliptic problem in this domain is not always straightforward, even if the shape of the interface is given. Theoretically, one can assume a shape for the interface, solve by using one boundary condition and then check that the other is satisfied. It seems reasonable to assume that there will be one solution where the two boundary condition will be met, thus, the interface will be determined. 


\section{Numerical implementation}

The numerical scheme for solving the diffusion equation involves adding a time derivative term, $T_{t}$, in equation (2.1), and time stepping the resultant equation. A steady state solution is reached when the derivative with respect to time, $T_{t}$, vanishes. For our boundary value problem, the signature of the initial conditions does not show up in the final solution, which should depend on the boundary conditions only. The insensitivity of the problem to initial conditions, and the important influence of different boundary conditions are verified by an array of numerical simulations.

Two dimensionless parameters are relevant in the analysis; the first, which was introduced already, is $\kappa_{i}$. This coefficient determines the vertical temperature profile that has a $\cosh \left(\pi z / \sqrt{\kappa_{i}}\right)$ dependence. In the south the vertical structure will be surface intensified, whereas in the north it will be approximately depth independent. The other parameter $\tau \kappa_{v}^{(i)} / D^{2}$, with $\tau$ as a time scale (say the number of seconds in a year) enters when the time derivative term is added, and is a measure of the rate of downward diffusion in the two regions. We would like it to be smaller than unity in the south, and bigger in the north. This would imply that diffusive effects penetrate deep and fast in the convective overturning regions, while they are more surface trapped in the stable regions. This last parameter is only important in time dependent cases; when the forcing varies with time or when the time dependent problem is investigated. When a steady state solution is reached, $T_{t}=0$, and no time scale is associated with the problem.

The numerical approach used is simple. All second derivatives are evaluated by a second order finite difference, while the grid configuration is such that the diffusion coefficient $\kappa$ is calculated in between points where the scalars $T$ (or $\rho$ ) are known. For example, if we let the index $i$ vary in the vertical direction, and let $C$ be any tracer quantity; then

$$
\left(\kappa C_{z}\right)_{z}=\kappa_{i+\frac{1}{2}}\left(C_{i+1}-C_{i}\right)-\kappa_{i-\frac{1}{2}}\left(C_{i}-C_{i-1}\right)
$$


where we took $\Delta z=1$. The same relation holds replacing $z$ by $y$ and letting $i \Delta y$ denote the distance in the north south direction. The diffusion coefficient above $\kappa_{i \pm \frac{1}{2}}$ is determined by looking at the sign of $C_{i+1}-C_{i}$ or $C_{i}-C_{i-1}$ correspondingly. If it is negative, the stratification is unstable and the coefficient is large.

Along the side and bottom boundaries no flux conditions are specified; thus, we create a grid point outside the boundary and equate the tracer value there to that at the point just inside the wall. This is equivalent to making the second order first derivative vanish there. It also enables us to easily evaluate the second derivative on all boundaries.

Practically, it is best to think of the grid configuration as a rectangular box with sides $\Delta y$ in the horizontal, $\Delta z$ in the vertical, and an average tracer concentration $C$. The change in concentration $\Delta C$ at each time step $\Delta t$ is due to the flux in and out of the box. Explicitly,

$\Delta C \Delta y \Delta z=\Delta t\left\{\Delta z\left[\left.\left(\kappa_{V} C_{z}\right)\right|_{\text {top }}-\left.\left(\kappa_{V} C_{z}\right)\right|_{\text {bottom }}\right]+\Delta y\left[\left.\left(\kappa_{H} C_{y}\right)\right|_{\text {right }}-\left.\left(\kappa_{H} C_{y}\right)\right|_{\text {left }}\right]\right\}$.

For our configuration, some simplifications apply. The horizontal diffusion coefficient $\kappa_{n}$ is a fixed constant everywhere, while $\kappa_{V}$ is determined from $C_{z}$ and thus is known at the top and bottom walls of each interior grid box. This means that the evaluation of $\Delta C$ is easily done at all points not bordering any of the walls. Grid boxes that share a wall with the boundary of our domain are treated in an almost identical manner. Along the Northern wall $\left.\left(\kappa_{H} C_{y}\right)\right|_{r i g h t}=0, \Delta y$ should be replaced by $\Delta y / 2$, because the grid boxes are only half as wide, while all other terms are known. Along the southern boundary, $\left.\left(\kappa_{B} C_{y}\right)\right|_{\text {left }}$ vanishes, and again $\Delta y$ should be replaced by $\Delta y / 2$. At the bottom boundary, $\left.\left(\kappa_{v} C_{z}\right)\right|_{\text {bottom }}=0$, and because the grid boxes adjacent to this wall are half the height, $\Delta z$ is replaced by $\Delta z / 2$. For the upper boundary, $\left.\left(\kappa_{v} C_{z}\right)\right|_{\text {top }}$ is specified, and again $\Delta z / 2$ replaces $\Delta z$. Along the four corner boxes, the same technique applies, taking note that their sides are $\Delta y / 2$ and $\Delta z / 2$. 
Writing the finite difference analogue to the diffusion equation, summing it up over all the domain-taking note that proper bookkeeping requires that an interior point represent twice as much area as a grid point along the wall and four times that of the corner point-shows that the net tracer input in each time step is the flux through the upper boundary. If this net flux sums to zero, say a cosine pattern, we neither gain nor lose tracer while running the model.

The stability criteria for determining the size of the time step is computed by the Von Neumann analysis, where a wave like solution $T_{j, k}^{n}=A_{n} e^{i(l j \Delta y+p k \Delta z)}$ is plugged into the finite difference form of the equation. In our notation, $n$ stands for a time step corresponding to an elapsed time $n \Delta t$ from the initial condition, $j \Delta y$ and $k \Delta z$ are the distances from the origin along the $y$ and $z$ directions, and $l$ and $p$ are the wave numbers in those directions. The numerical scheme is usually stable for all wave numbers when the amplitude of the wave $A$ does not increase with time, so $A_{n+1} / A_{n} \leq 1$. This leads to an upper bound on the time step

$$
\Delta t \leq \frac{1}{2\left(\frac{1}{\Delta y^{2}}+\frac{\kappa}{\Delta z^{2}}\right)} .
$$

For most practical implementations, the limit is usually smaller.

The physical basis behind the size of the time step is that the information will not travel more than one grid point away at each step. This way there is enough time for each point to adjust itself to changes in the fields. In our diffusive problem, this translates to a scale determined by dividing the diffusion coefficient by the squared grid spacing. 


\section{Results}

\section{Vertical mixing with specified fixed flux}

Numerical simulations were used to check a wide range of initial conditions, surface heat fluxes, and parameter ranges. For the linear problem (2.1) one should not expect difficulties, especially because a simple analytic solution exists (2.2). This solution is reproduced numerically in figure $(2.2) \mathrm{a}$; where $Q=\cos (\pi y), \quad y \in[0,1]$, and $\kappa_{1}=$ $\kappa_{2}=1$, which according to our previous analysis is a reasonable value for oceanic scales. Further numerical experiments show that the nonlinear problem (2.4) behaves in the same desirable fashion and seems insensitive to different initial conditions, always resulting in the same final temperature structure, regardless of the initial temperature distribution pattern.

As we start increasing the ratio $\kappa=\kappa_{2} / \kappa_{1} \quad[Q=\cos (\pi y)$ as before $]$, the temperature contours in the northern region tend to become vertical while cooling persists all the way from top to bottom. The transition from the pattern suggested in figure (2.2) is smooth. The solution for $\kappa=\kappa_{2} / \kappa_{1}=100$ is represented in figure (2.2)b. The heavy marked line is the border between the convective overturning region and that with a thermal diffusivity $\kappa_{1}$. The turbulent region where mixing is controlled by convective overturning is always present in places where heat is taken out of the ocean into the air, so that in all regions where $Q<0$ at the surface, vertical mixing is dominant, and the well mixed region might reach all the way to the bottom. The boundary lines surrounding the strong vertical diffusion region always emanate from the $Q=0$ points at the surface.

In the two other figures, the ratio $\kappa$ was still kept 100 but $\kappa_{1}$ is no longer a unity as it is in (2.2)a-b. For (2.2)c $\kappa_{1}=10$, while for (2.2) $\mathrm{d} \kappa_{1}=0.1$. Increasing $\kappa_{1}$ corresponds to stronger vertical diffusivity in the south, while decreasing it is equivalent to a more pronounced horizontal diffusivity. This is born out clearly in the pictures. The temperature structure in the south is more surface trapped when the horizontal 

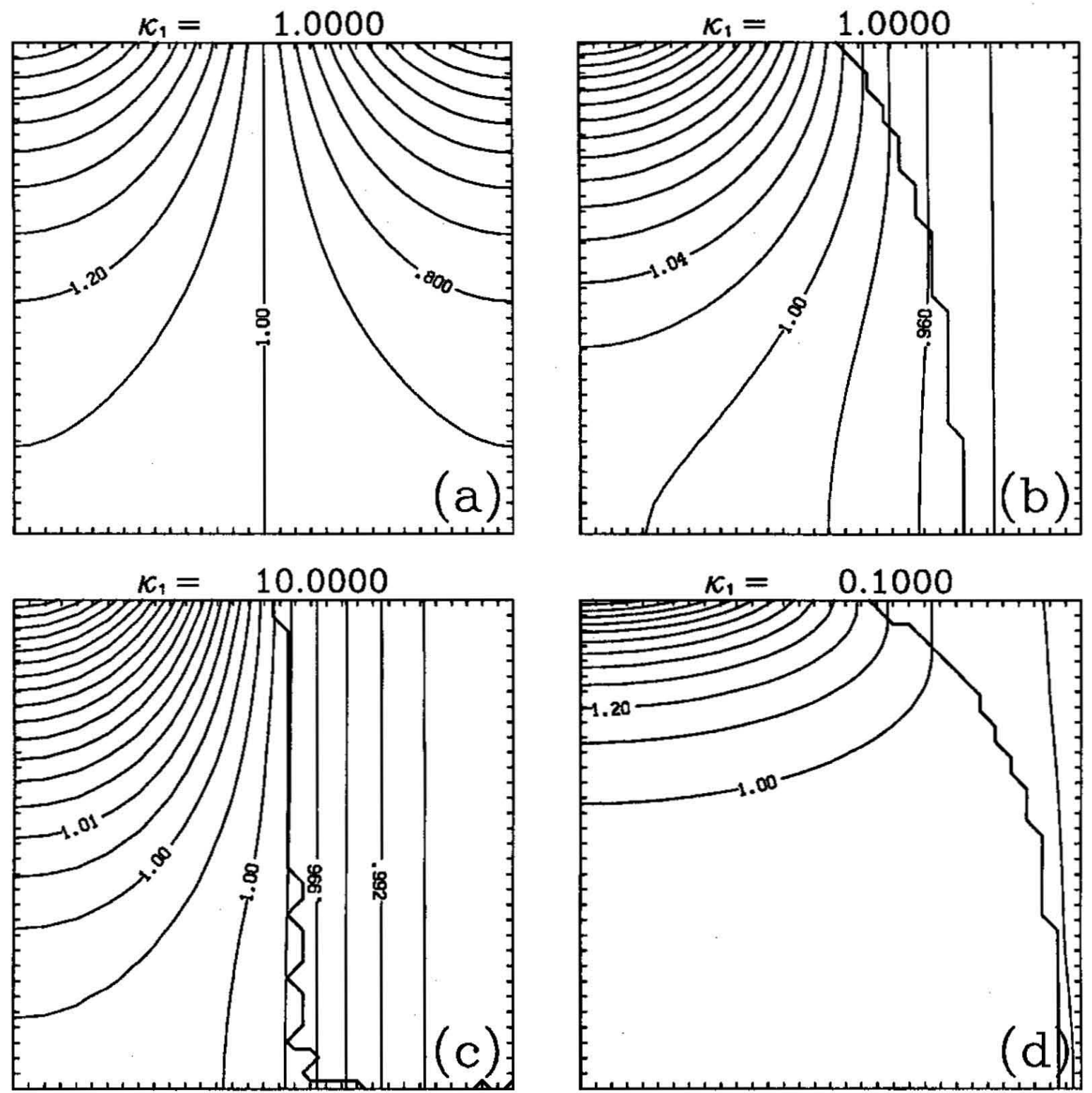

Figure (2.2). Temperature structure from solving equation (2.4) for a specified $\cos (\pi y)$ heat flux. The ratio $\kappa$ between the diffusivities in the northern $\left(\kappa_{2}\right)$ and southern $\left(\kappa_{1}\right)$ regions is 1 in figure (a) and 100 elsewhere. (a) $\kappa=\kappa_{1}=1$, as in the analytic solution (2.2) with no overturning. (b) $\kappa_{1}=1$, regions to the right of the heavy line are weakly unstable. (c) $\kappa_{1}=10$. (d) $\kappa_{1}=0.1$. 
diffusivity is strong (2.2)d, and less so [compared to (2.2)b] when the vertical diffusivity is dominant (2.2)c. In the north, the temperature is always nearly depth independent.

As the pictures indicate, the shape of the interface is sensitive to the value of $\kappa_{1}$. Similar numerical experiments that are not presented here, were carried out with the same values of $\kappa_{1}$, but with larger values of $\kappa=1000$ and 10000 . These experiments verified our previous statement that for fixed $\kappa_{1}$, the shape of the interface does not depend on the ratio $\kappa$. The choice of $\kappa=100$ is based on practical considerations. Because the time step is inversely proportional to $\kappa(2.7)$, a steady state solution for large $\kappa$ will take considerable computer resources. Since we already argued that to first order neither the interface nor the temperature in the south depends on it, one can safely choose values which are not very large. On the other hand, the figures clearly show that the location of the interface depends on the relative strength of horizontal and vertical diffusivities in the south. The stronger the horizontal diffusivity, the more the interface is pushed at the bottom toward the northern wall.

After exploring the sensitivity of the model to different diffusivities we look at the effects of the boundary conditions. Two types of surface heat fluxes are treated.

1) A specified value corresponding to $\kappa T_{z}$. This value might be time dependent.

2) A flux that is proportional to the difference in temperatures between the sea surface and that of the air. This flux can vary with time even if the air temperature is fixed because the sea surface temperature can be time dependent.

In order to reach a steady state, the total amount of heat exchanged between the ocean and atmosphere should be zero. For the first case this translates to the integral constraint $\int \kappa T_{z} d y=0$. (To avoid possible problems in northern regions where the diffusivity can change in time due to local instability, the local surface heat flux $\kappa T_{z}$ is always specified instead of specifying $T_{z}$ and multiplying it by the appropriate $\kappa$.) If the flux is time dependent, a periodic steady state can be reached, namely, a situation where there are periods of time over which the cumulative flux vanishes. We can write 
this constraint as $\int d t \int T_{z} d y=0$, since, although in the winter the oceans get colder and in the summer warmer, on a yearly (or multi yearly) average, their heat content is fixed in this approximation.

Whichever forcing is chosen, the convective overturning is limited to regions of negative heat flux. In addition, the shape of the solution in the southern region always approximates that suggested in the analytic solution (2.2). If the cooling period is short compared to the speed of the downward vertical mixing, the marginally stable region can be bounded vertically, and will not reach the bottom.

\section{Forcing with a yearly cycle}

Results from a periodic steady state based on monthly average data are represented in figures (2.3)a-e (the results repeat in a yearly cycle). The data, taken from Oort and Vonder Haar (1976), was stretched from their $10^{\circ}$ latitude belt to fit our grid resolution. As the authors point out, there are several problems associated with this data, including the less than reliable information in the northernmost latitude belt, and the fact that the data really relates to fluxes in and out of a latitude belt which covers both land and ocean, while the rate of heat storage is smaller for land than for water. We will not go into these difficulties, nor do we claim that our results are an exact representation of the oceanic heat balance.

One should view our specified monthly heat balance in more general terms, and consider only the broad similarities between our results and ideas about the general circulation. In particular, one should note, that in the data collected by Oort and Vonder Haar the annual average rate of energy flow from the atmosphere to the ocean in the latitude band $60^{\circ}-90^{\circ}$ is positive. In our results this reflects itself as warming so the temperature structure in the north is somewhat like the south. It is probably best to limit our observations to latitudes below $60^{\circ}$ where the data is more reliable. The other parameters used in the numerical simulations were $\kappa=100$ and $\tau \kappa_{v}^{(1)} / D^{2}=0.02$ 

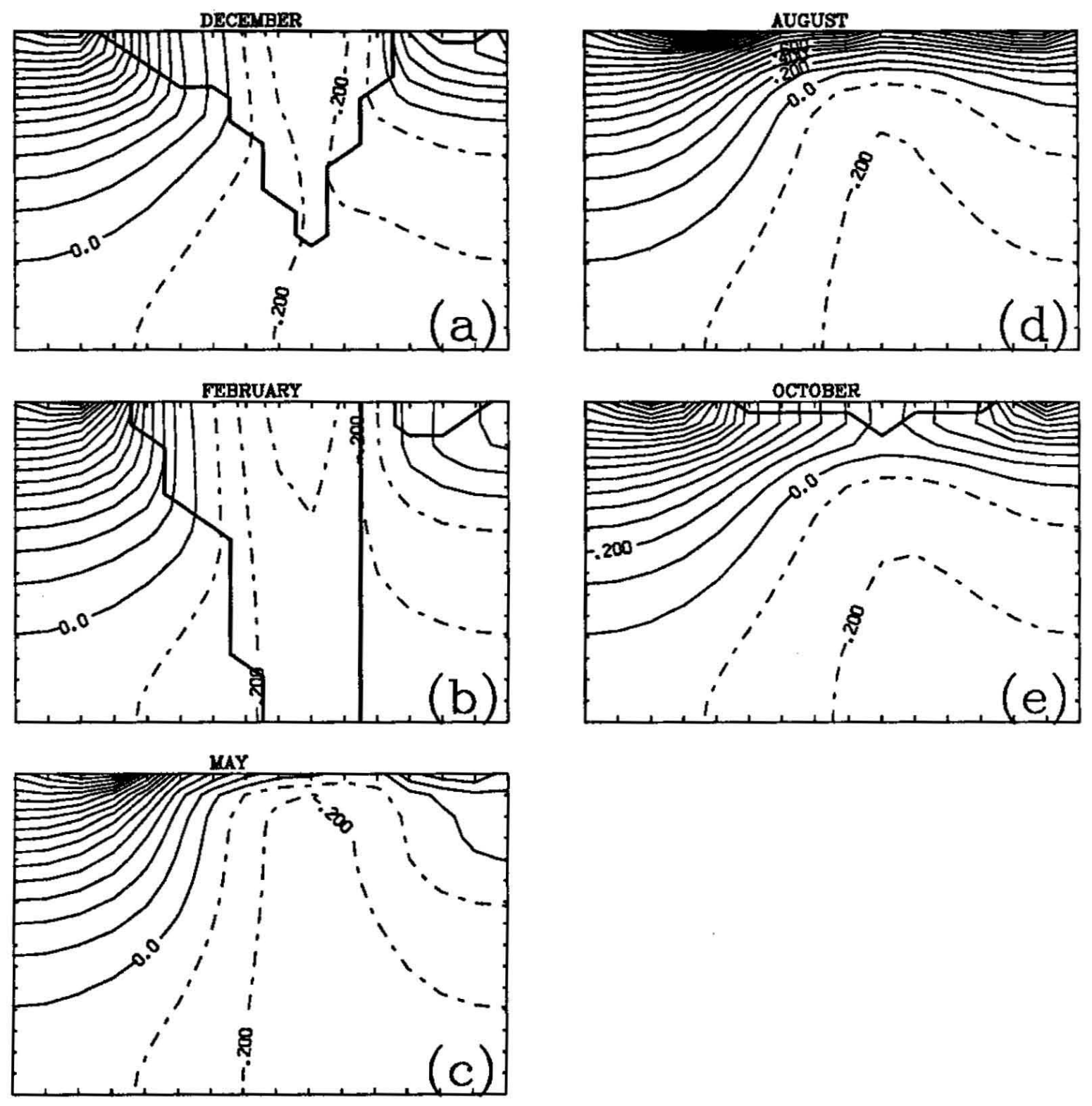

Figure (2.3). Temperature structure in a periodic steady state based on monthly varying heat flux taken from Oort and Vonder Haar (1976). (a) December. (b) February. (c) May. (d) August. (e) October. 
The first figure, (2.3)a, corresponds to December, when the unstable region is quite deep but does not reach all the way to the bottom. The large surface extent of the unstable region is a result of cooling over a large latitude band. This is due to warm surface waters and cool air, typical of late summer/early winter situation. As winter progresses, the region deepens, reaches the bottom and becomes wider. Figure (2.3)b shows the maximum extent of the convective overturning region occurring at the end of February, when the unstable region covers most of the subpolar gyre. As summer starts, the unstable region is isolated from the top, because $Q$ suddenly becomes positive. The blob of fluid with $\kappa_{2} \gg \kappa_{1}$ shrinks and erodes very rapidly due to horizontal diffusion. In the ocean, the decay rate is sometimes slower. For example, the signature of the Weddell polynya can be detected many months after its creation. But much like in other eddy features, this is probably due to mechanisms not treated here, most notably, the flow field and the relative stability caused by possible rotation of the chimney. Other structures like the MEDOC chimney (see chapter 3) erodes faster, on order of a few months. May is the first month in which the stratification is stable everywhere [figure (2.3)c]. Note, that the temperature structure in southern regions does not change. As summer progresses, the temperature structure becomes more surface intensified. Compare, for instance, the zero contour for May and August [figure (2.3)d]; the latter is much deeper as a result of three months of intense heating. In October the heat flux changes sign, and much of the north is cooled. Unstable regions evolve, and a chimneylike structure can be seen between the 0.0 and the 0.1 contours of figure (2.3)e in the region of the lowest point of the interface. The model developed in the next chapter will deal with this feature more extensively. Note also, that the deep fluid is relatively unaffected by the seasonal cycle, and does not show any strong horizontal or vertical gradients. In addition, in all realizations the isotherms bend upward from the equator and the northern boundary into the subpolar gyre. In spite of the simplicity of the 
description, we are able to capture some of the general features of a yearly climatological cycle in the world ocean by employing the simple idea of convective overturning.

\section{Flux depending on air to sea surface temperature gradient}

When the surface heat flux is proportional to the difference between the air-sea temperature, our results are qualitatively the same, but require some elaboration. For simplicity, assume that the air temperature has a $\cos (\pi y)$ (constant in time) profile, and let $Q=T^{\text {air }}-T^{\text {sur }}$ (up to some proportionality constant which does not depend on the value of $\kappa$ next to the surface). In a steady state, the temperature of the fluid does not change, so $\int Q d y=0$, or $\int T^{\mathrm{sur}} d y=0$, but as the solution evolves towards this condition, there are changes in the temperature structure. Let us use the analytic solution (2.2) as an initial condition, so that the surface temperature is symmetric in $y$ and averages to zero. As time progresses, the changes in southern regions are small compared to those in the north, (this is of practical importance, since the time step is governed by the largest of the $\kappa$ 's, although changes in the south happen on scale of the smaller one) where the surface temperature tends to be more homogeneous because the whole water column is being cooled, as opposed to a surface intensification in the south. This leads to the relation $\int T^{\text {sur }} d y>0$, which is observed in figures (2.2)b and (2.6)a. As a result, $Q$ is negative, and the fluid is cooled more and more as time goes by. As already noted, we must end up with $\int T^{\text {sur }} d y=0$, and since we started with a value of zero and progressed to a positive one, we must pass some maximum along the way, after which the value of the integral decreases to zero ever so slowly. It is around this maximum that the solution has actually gained its final shape, which is basically kept intact (apart from a decrease of temperature everywhere) as $\int T^{\mathrm{sur}} d y \rightarrow 0$. Figure (2.4) shows the relationship between the air and sea surface temperatures in the 
process, with figure (2.4) $\mathrm{b}$ as an enlargement of (2.4)a around the intersections of the four lines.

Line (A) is the air temperature which is fixed in time and has a cosine profile. (B) is the initial surface temperature-also with a cosine profile so $Q(t=0)=0$. (C) is the surface temperature after some time-note that it has a positive integral so $Q(t>0)<0$. Line (C) is different than (B) in the northern region only, indicating that the convective overturning process is faster than the regular mesoscale mixing in influencing the stratification. Over this short time span, the surface temperature in the north decreased by a couple of degrees Celsius, while that in the south remained fairly constant. (D) is the final surface temperature profile. It is almost like (C), but is shifted down along the ordinate over the whole domain. This results in a more horizontally uniform temperature structure, where now the integral of the surface temperature vanishes. The surface location of the $Q=0$ point drifts slightly to the north. Initially it is at the $y$ coordinate of 8.45 (intersection of lines A and C), while in the final stages the intersection of lines $\mathrm{A}$ and $\mathrm{D}$ is at $y=8.65$. Figures (2.5) $\mathrm{a}-\mathrm{b}$ show the isotherms for $\kappa_{2} / \kappa_{1}=100$ at two different times. Figure (2.5) b is the result of running 50 times longer than was necessary for (2.5)a, and both after a long enough time so that the integrated surface temperature converges to zero. Note, that not only do they share the same general structure (apart from the fact that the average temperature decreased), but also that both look remarkably like figure (2.2)b for which we used the same $\kappa$ but a cosine heat flux.

Differences between the case with specified air temperature and that with specified flux can be identified by looking at the horizontal temperature structure at three different depths presented in figures (2.6)a-b. The first corresponds to three cross sections in figure (2.2)b, and the second to the same sections in figure (2.5)b. Section (A) is the surface temperature, (B) the temperature two grid points away from the surface $(13 \%$ 

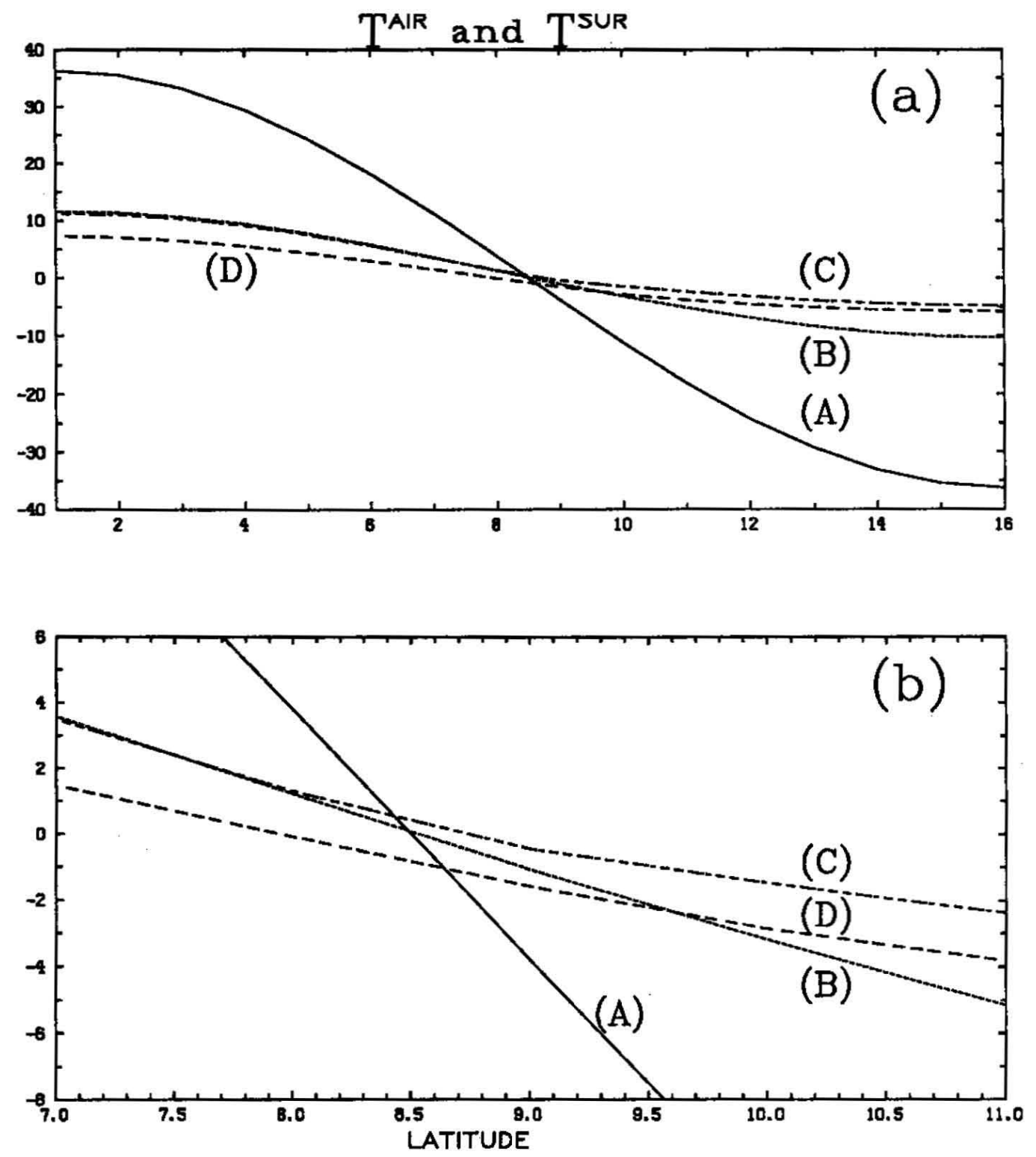

Figure (2.4). The relation between the $T^{\text {air }}$ and $T^{\text {sur }}$ when only the first is given and the flux is proportional to the difference between them. Line (A), the air temperature. Line (B), the initial surface temperature. Line (C), the surface temperature after some time. Line (D), the final surface temperature. (b) is an enlargement of (a) around the intersection of the four lines. 

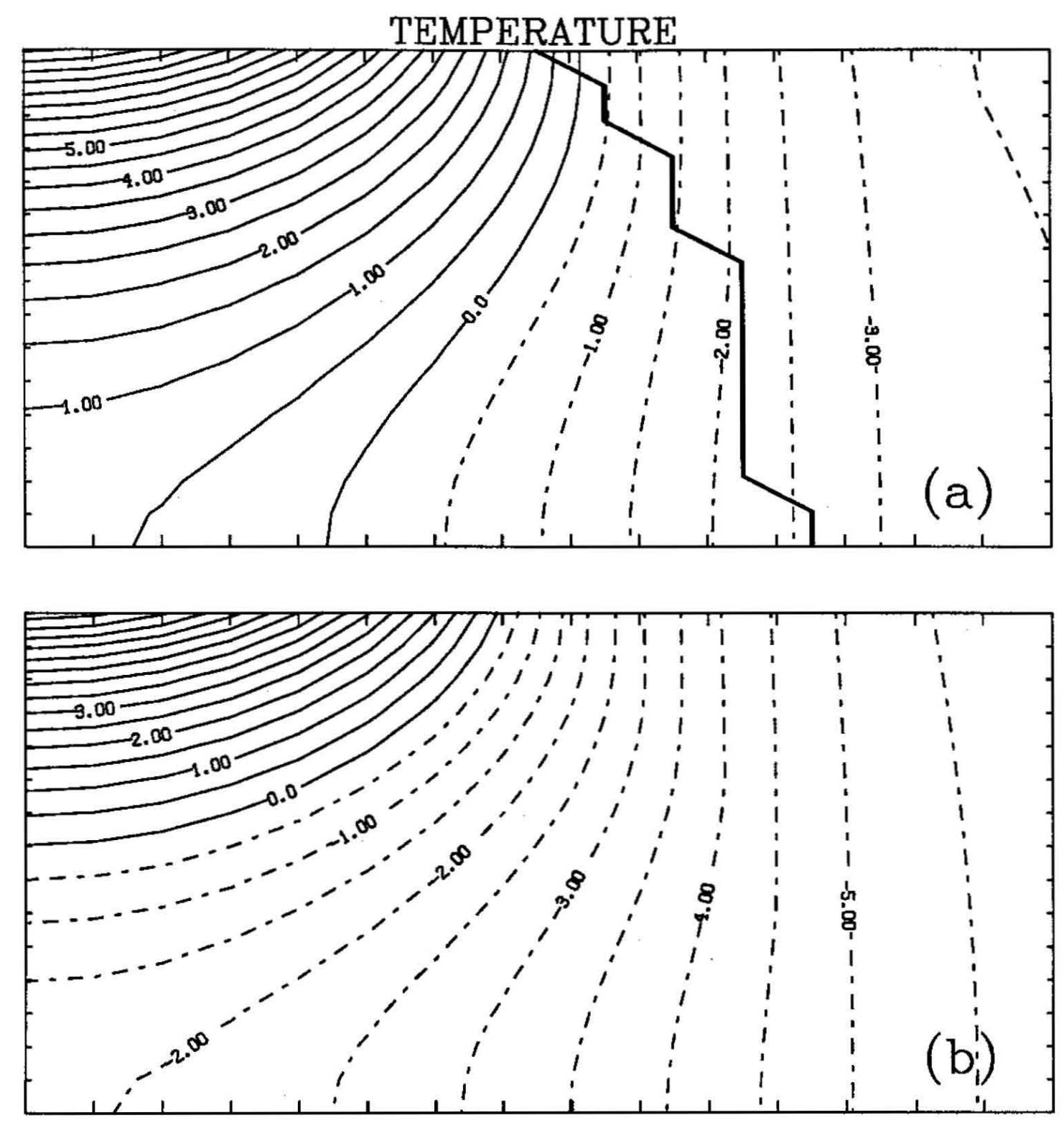

Figure (2.5). Temperature structure when the flux depends on the specified air temperature. (b) is the same as (a) but after running the model 50 times longer. 
of the total depth), and (C) is the temperature one grid point from the bottom (7\% of the total depth from the bottom).

It is evident that the temperatures in figure $(2.6) \mathrm{b}$ are everywhere colder than in (2.6)a. This is due to the fact that for a specified air temperature a steady state is reached only after intensive cooling, whereas for a specified flux, the net cooling is always zero. But the temperature gradients are also different in the two cases. The horizontal temperature differences in $(2.6) \mathrm{b}$ are $3.5,9.75$, and 12.75 corresponding to lines (C), (B), and (A), while they are about 1.2 times larger in figure (2.6)a. The vertical temperature differences also exhibit the same tendency. The surface temperature is about $11.5^{\circ} \mathrm{C}$ warmer than the near bottom temperature in (2.6)a, while it is only $9^{\circ} \mathrm{C}$ warmer in (2.6)b. This indicates that not only is the fluid everywhere colder when the flux depends on the air to sea-surface temperature, but also that the stratification as well as the horizontal gradients are weaker. The figures also help corroborate some of the observations we have already made. In northern regions all three plots merge to almost a single line, indicating that the temperature is vertically uniform. The horizontal thermal structure is more pronounced near the surface, confirming our observation that the deep fluid is cold and nearly homogeneous, while the temperature structure is surface intensified. 

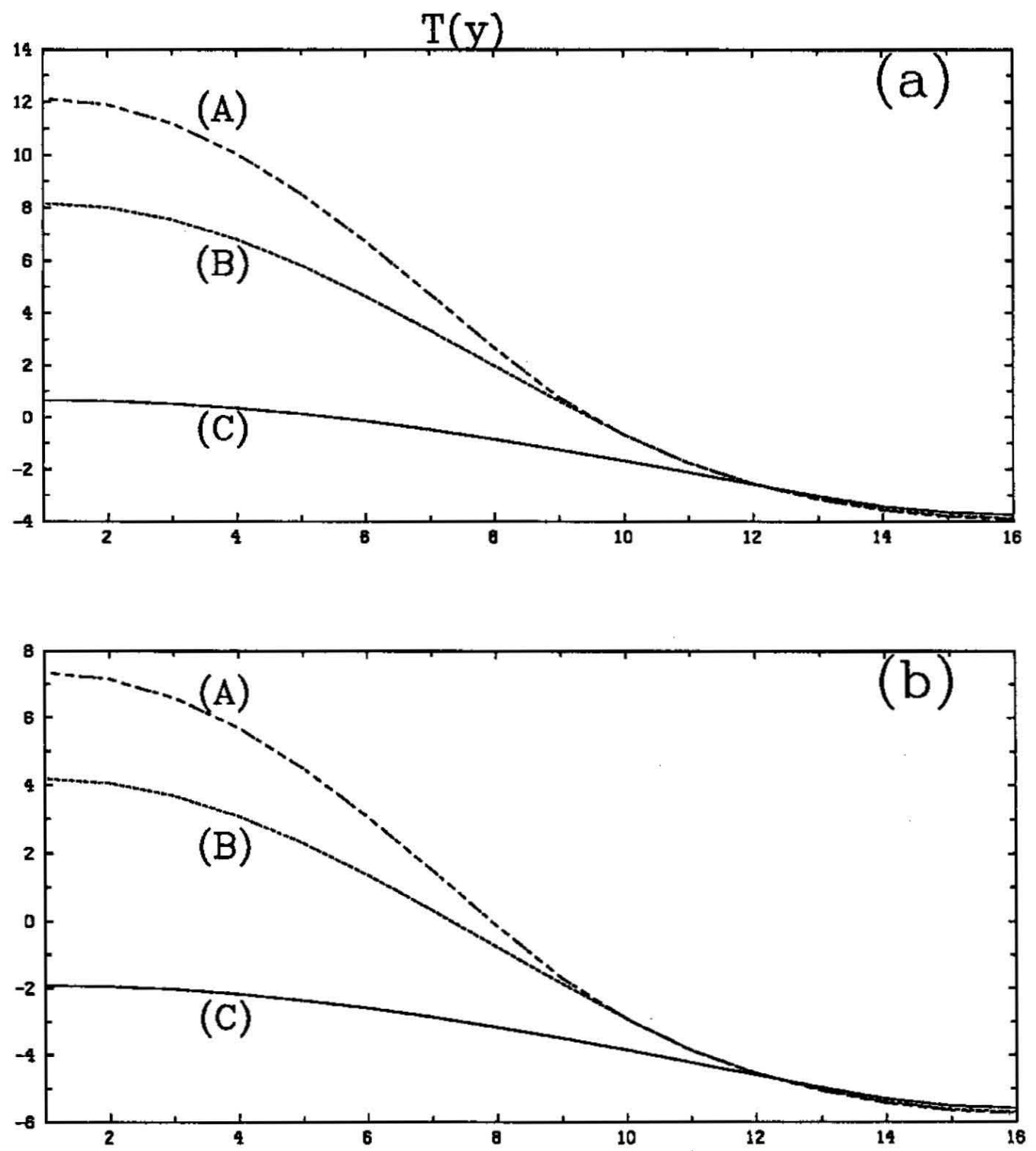

Figure (2.6). South-north temperature cross sections at three different depth. Line (A), surface temperature. Line (B), $13 \%$ of the depth from the surface. Line (C), $7 \%$ of the depth from the bottom. (a) Corresponds to figure (2.2)b. (b) Based on data taken from figure (2.5)b. Note the different vertical scales. 


\section{Different averaging schemes}

The last set of figures for the convective overturning model (2.7)a-c shows the difference between a steady state resulting from forcing the fluid with a yearly average of a monthly varying heat flux, and that resulting from taking a yearly average of monthly results. In figure (2.7)a we have taken a simple average of the temperature structure on the last day of each month, where the monthly states were computed using the Oort and Vonder Haar (1976) data mentioned before. Out of these twelve monthly states, five were already introduced in figures (2.6)a-e. Figure (2.7)b is a result of taking the average of the monthly varying heat flux and generating the appropriate steady state. For both these figures $\kappa=100$ in the unstable regions, but is taken to be one in figure (2.7)c, which in all other respects is the same as (2.7)b. Therefore, the relation between (2.7) $\mathrm{c}$ and (2.7) $\mathrm{b}$ is the same as between (2.2)a and (2.2) b, with the sole exception that the heat flux used is different. The region between the heavy lines in (2.7)c is the unstable region.

As expected, all three figures have the familiar $\cosh (z) \cos (y)$ temperature structure in the south where the isotherms are surface intensified. But even there it is clear that the surface temperature in (2.7)a is higher than in the other two figures. This phenomena is a result of convective overturning. In the winter-for instance the month of December in figure (2.3)a-the convective overturning region reaches well into the southern portion of the basin, thereby mixing the upper cold fluid with warmer water from below, and on the average cooling the surface water less. Thus, the average temperature is warmer than that corresponding to the average heat flux. On the other hand, in figure (2.7)b the overturning region is fixed, and never gets close to the equator. This warming effect of turbulent mixing can be found in other locations, as can be seen by comparing the northern regions in (2.7) b to (2.7)c, and (2.2)a to (2.2)b. One can also explain the surface warming by looking at the differences between the yearly cycles with and without overturning. If there is no intensive mixing the temperature 

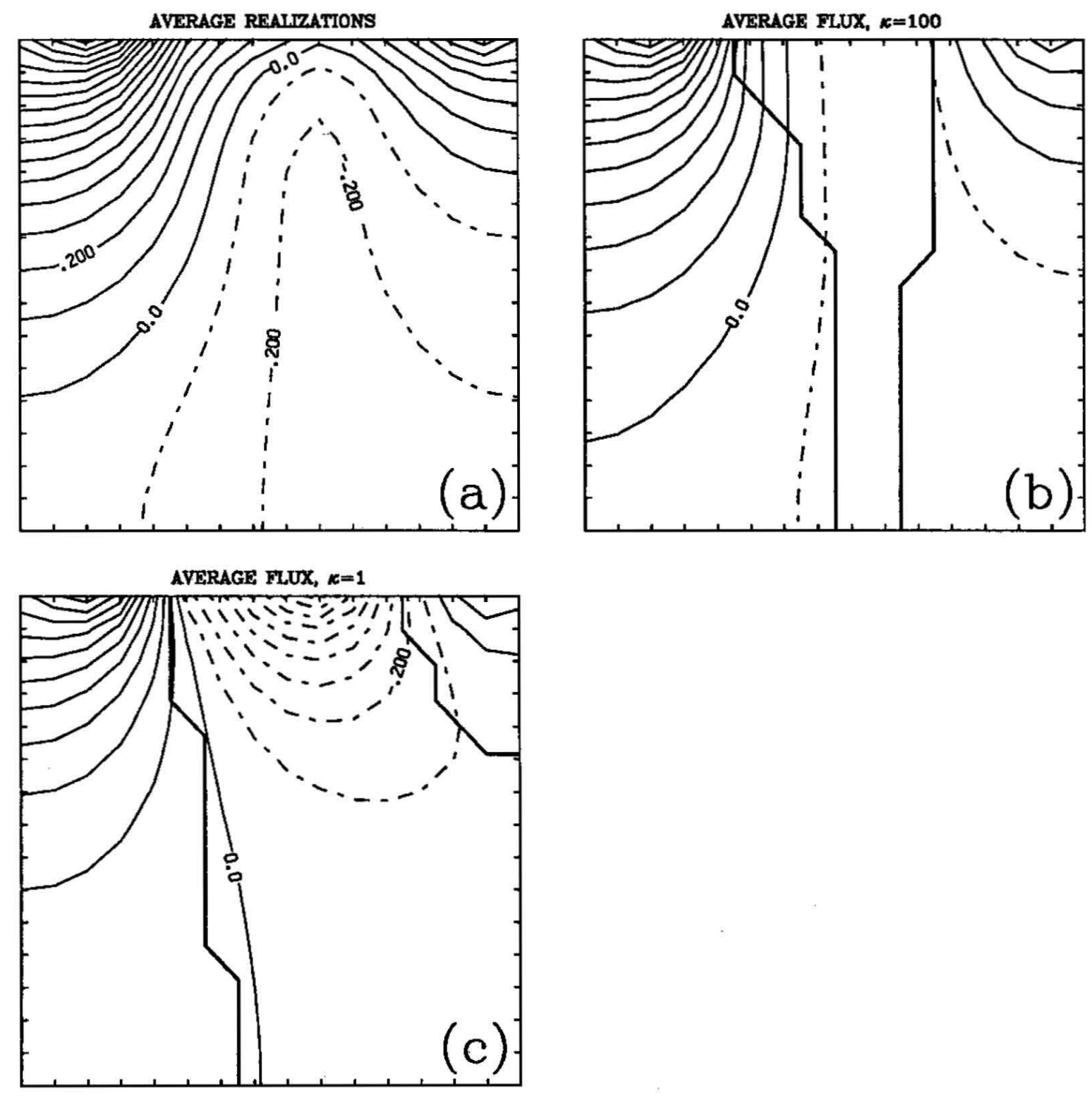

Figure (2.7). Temperature structure for different averages with the monthly data taken from Oort and Vonder Haar (1976). (a) Average of twelve monthly realizations, each with $\kappa=100$. (b) A steady state from average heat flux of the above data with $\kappa=100$. (c) The same as (b) but $\kappa=1$. 
only changes seasonally next to the surface, and is approximately fixed at great depth. It there is mixing, the situation is different in the cooling periods. At that time the mixing penetrates deeper than before, while the temperature of the water column next to the surface is almost depth independent. Its value is some mean of the cold surface water and the warmer water from below, thus necessarily warmer than without mixing.

The other major difference between the figures is of course the colder regions where we see that although the average heat flux results in a convective overturning region, the monthly average does not. Note also, that the overturning region does not have large horizontal gradients, especially compared with the unstable region in the linear problem whose solution is presented in (2.7)c. As with most of our results so far, the deep fluid is nearly homogeneous regardless of method of averaging.

\section{Discussion}

After having established the insensitivity of the numerics to initial conditions, the influence of different heat fluxes on the final temperature structure is explored. Several features are observed in all the results.

The structure in southern regions, where the fluid is heated, exhibits a strong resemblance to the surface intensification property of the analytic solution (2.2). In northern regions, where the heat flux cools the ocean, the convective overturning process dominates, and the isotherms are nearly vertical. The deviation from vertical is due to the finite vertical diffusion coefficient. The larger it is, the more vertical the lines become, but practical considerations of decreasing time step with $\kappa$, and a lack of new insight for very large $\kappa$ 's, dictates choosing a moderate diffusion coefficient. The interface between the two regions emanates from points at the surface where the flux vanishes. Its shape is controlled by the strength of the horizontal diffusion in the south. 
The periodic simulations clearly show that a yearly cycle can be reproduced with convective overturning. Again, the features in northern and southern regions are practically unchanged year-round, but those in mid-latitudes show a pronounced response to the thermal forcing. The yearly variability of the density field is largest at places where the turbulent processes are strong, in particular, if the changes of the forcing with time are large. The cooling in the winter time produces a region with weak vertical gradients. This region is isolated from the top at the beginning of the summer, and erodes by horizontal diffusion. The picture described above gives a rough sketch of the oceanic temperature structure. There is a thermocline in southern regions, cold deep fluid everywhere, and weak vertical gradients in northern regions. These gross features are apparent in the time dependent and steady state realizations.

\section{Flux versus air to surface temperature gradient}

For the linear problem (2.1), specifying the flux as proportional to the temperature difference between the air and the surface fluid is equivalent to a mixed Neumann and Dirichlet boundary conditions, resulting in a well posed problem. The nonlinear problem is probably still well posed, but the already noted remarkable similarity between figures (2.2)b and (2.5)b, opens some new questions about the difference between cases where the heat flux is given and those with known air temperature. The exact details of the time development of the two until a steady state is reached was summarized in the previous section.

It is easy to see that if $T_{1}(y, z)$ is a solution for a given $T_{1}^{\text {air }}(y)$, then $T_{2}(y, z)=$ $T_{1}(y, z)+T_{0}$ is a solution when the boundary condition specified is $T_{2}^{\mathrm{air}}(y)=T_{1}^{\mathrm{air}}(y)+$ $T_{0}$. This statement is an indication that changing the air temperature by a constant will change the solution by the same constant everywhere. In this respect the air temperature boundary condition differs drastically from the specified flux boundary condition. For the latter, if its integral over space and time is not zero, there is a net 
cooling or heating, and no steady state is possible. For the former, the steady state is possible because the surface temperature can always adapt itself so that the integrated gradient of air-water temperature vanishes.

For example, line $\mathrm{A}$ in figures (2.6) a and (2.6)b corresponds to the surface temperature structure in figures (2.2) b and figure (2.5) b, both with a cosine-like shape (at least in the south). But there is a notable difference between the two: when we specify a cosine flux structure, $T^{\text {sur }}=0$ at mid-latitudes $[8.45$ on the figure (2.6)a]; when the air temperature is given, the surface temperature at the same location is about -1 units in figure $(2.6) \mathrm{b}$. In this case, there is a net heating at that point, and the line separating the convective overturning region emanates from a point more to the north than before. The same difference is observed in figure (2.4) lines (A) and (D) as explained in the previous section. An even simpler example is given by the case in which the air temperature is, say, $2^{\circ} \mathrm{C}$ everywhere, the steady state is an ocean with a uniform temperature of $2^{\circ} \mathrm{C}$, and hence no flux anywhere. The corresponding flux boundary condition is zero flux everywhere, so diffusion can smear all temperature gradients. For a flux condition, only if the average temperature of the fluid is initially $2^{\circ} \mathrm{C}$, will the final temperature be $2^{\circ} \mathrm{C}$; for different initial temperatures, the final temperature will be a different constant.

This last observation has a numerical application: for a given air temperature, one can compute $\int_{0}^{L} T^{\text {air }} d y=\mathcal{H}$, (with $L$ as the south-north extent of the domain) and then subtract $\mathcal{H} / L$ everywhere from $T^{\text {air }}$. In this way we can conveniently work with numbers with a zero average.

Since the flux and air temperature boundary conditions react differently to a constant shift in forcing, the striking resemblance of figures (2.2) b and (2.5)b may not be a general rule. Thus, if there is a solution to the problem where the flux depends on the difference between the specified air temperature and the sea surface temperature 
that is determined by the model, it is not always possible to specify a simple heat flux that will give the exact same temperature everywhere.

Consider the two solutions to the linear diffusion equation (2.1) with the same $\kappa$ and with $z=0$ at the bottom and $D$ at the top. Let the first, $T^{(1)}$, be reached when the specified heat flux at the surface is $\kappa T_{z}^{(1)}(y, D)=-Q(y)$, and the second, $T^{(2)}$, corresponds to the case with the flux depending on the difference between the surface temperature and the specified temperature outside this boundary, $\kappa T_{z}^{(2)}(y, D)=T^{(2)}(y, D)-Q(y)$, where in both cases $Q=\left(Q_{0} / \sqrt{\kappa}\right) \cos (m \pi y)$. The analytic solution (2.2) to the linear Poisson problem is $T=A(m) \cos (m \pi y) \cosh (m \pi z / \sqrt{\kappa})$, so for the two different boundary conditions we get

$$
\begin{aligned}
m \pi \kappa A^{(1)}(m) \sinh (m \pi D / \sqrt{\kappa}) & =-Q_{0} \\
m \pi \kappa A^{(2)}(m) \sinh (m \pi D / \sqrt{\kappa})-A^{(2)}(m) \cosh (m \pi D / \sqrt{\kappa}) & =-Q_{0} .
\end{aligned}
$$

From these expressions we can find the coefficients $A^{(1)}(m)$ and $A^{(2)}(m)$, and use them in the expression for $T$ above. For large enough $m$ or $\kappa$, we can neglect the second term in the last equation compared to the first, and thus we have $A^{(1)}=A^{(2)}$. The introduction of convective overturning in (2.4) turns the problem into a nonlinear one. However, if we use the same argument-which should strictly apply only to the linear system-and apply it in our example for which $\kappa=100$ and $m=1$, we find that the two solutions should be quite similar. The constant difference in the temperature between the two figures is of no concern, because we can always add free solutions to the homogeneous problem-like a constant.

For smaller values of $\kappa$ the second term in (2.8) cannot be neglected, and the differences between the results are more pronounced than those between figures (2.2)b and $(2.5) \mathrm{b}$. 
Steady state versus average of periodic states

In the oceanographic literature many studies are based on the usage of climatological forcing, in particular, the use of climatological winds to force the oceanic circulation. Obviously, climatological quantities are nothing but a mean over instantaneous measurements, and the larger the time span of the measurements, the more indicative of climatology they are. Other climate models use seasonally varying forcing as boundary conditions; for example, Busalacchi and O'Brian (1980) and Philander and Pacanowski (1984). Most of the recent studies are done using primitive equation general circulation models applied in tropical regions. This is due to the fact that people believe that the response of the equatorial circulation to variability is much stronger than that of mid-latitudes. But in general, those models say very little on the difference between the mean of the variable forcing and the climatological one.

The purpose of figures (2.7) $\mathrm{a}-\mathrm{c}$ is to show the difference between forcing the ocean by a time dependent force and looking at the mean temperature structure, and forcing the ocean with a time average forcing. A major difference between these two approaches is that the last is a true steady state, while the first is a composite of time dependent situations. A priori, this is sufficient for the two techniques to yield different results, in particular because our problem is inherently nonlinear.

Based on changes occurring in both the cold and warm regions, several important features can be established from the three figures. When averaging periodic states, the surface temperature is warmer in the south and the surface intensification is more pronounced. This change is mainly due to the fact that in taking the average of monthly realizations, because the surface heat flux is time dependent, the influence of the turbulent mixing can be traced in regions where in the second average we have only heating. This is particularly true for the southern regions, where the mixing is vigorous in the winter time due to cooling, but the net yearly effect is heating. As was explained before, convective overturning in the south in the cold winter months 
will cool the surface water less due to heat exchange by vertical mixing with deeper fluid. This would change the stratification up to the penetration depth of the winter processes, and make the surface temperature intensification more pronounced in figure (2.7)a than (2.7)b.

Similar observations where also made by Bryan and Lewis (1979) when running a primitive equation general circulation model. The strategy for their numerical experiments had three parts. An initial spin-up period of about 521.6 real years-where the fluid was forced with an annual mean forcing-was followed by about 604.5 real years with a seasonal forcing. The final conditions at the second stage were used as a basis for a variety of relatively short experiments for tuning of parameters. The authors note (see also figure 4 in their article) that when the ocean is forced by seasonal boundary conditions the interior temperature decreases and the thermocline becomes shallower. This is in qualitative agreement with the results presented in this section and those of chapter 5.

This observation is particularly important in ocean-atmosphere models where it is common to force the fluid with climatological forcing as in figure (2.7)b, in particular those models that use convective overturning adjustment. If the first averaging procedure is used, the surface temperature in equatorial regions is warmer by about $4^{\circ} \mathrm{C}$, thus, having a drastic effect on the heat flux and the related results.

Another striking feature is the lack of a convective overturning region in figure (2.7)a. The reason behind this is very simple. If there is one month in which the stratification is stable everywhere, then adding this stratification to the yearly average marginally unstable stratification will result in a stable structure. This is true because the turbulent mixing process weakens the vertical temperature gradients, thereby making what was initially unstable become only marginally unstable. If we add these very small negative gradients to any positive gradient representing a stable stratification we will most often end up with a positive quantity. Indeed in the summer time figures 
(2.3)c-d indicate that the oceans extracts heat from the air at all latitudes from equator to pole, thus, positive vertical temperature gradients can easily overcome any weak winter time negative gradients.

This would not be true in the absence of convective overturning. In this case, the strength of the instability would be comparable to the stabilizing effects, and the net effect at each location would depend on whether cooling or heating was more persistent there. Because of the lack of overturning region in (2.7)a, the isotherms in the north are not vertical next to the surface as in (2.7)b, and the temperature is warmer in the north. This means that although the surface temperature in equatorial regions is higher, $T_{y}$ (which is needed when computing the meridional heat flux in the ocean) can be the same or even smaller.

The last figure (2.7)c is again a result of heating by average forcing, but this time with no turbulent mixing ( $\kappa=1$ everywhere). It shows clearly two of the main characteristics of the mixing process that are also be observed in chapter 5 where circulation is included in a similar model. The unstable region is smaller when mixing is introduced, indicating that the process is successful in bringing most of the fluid to a stably stratified state. The temperature is also different. The mixing process homogenizes the temperature in the vertical, and at the same time it makes the interior colder by bringing cold surface water in the north downward. Compare for instance the 0.0 contour and see that a larger portion of the fluid is colder in (2.7) a-b than in (2.7)c. As a side effect, because the net amount of heat in the system is fixed, the temperature contours next to the surface are a bit more crowded with mixing than without. In chapter 5 the distinction between this crowding of isotherms and the depth of the thermocline will become clearer. 


\section{Conclusions}

We have used a mixing parameterization that assumes that turbulent mixing is dominant over mesoscale mixing in unstable regions. This parameterization was combined with the assumption that the flow field is very weak, (diffusive fluid), and proves successful in capturing some features observed in the general circulation pattern. We generate a thermocline in southern regions, deep cold fluid everywhere, and vertical homogeneity in the north. These features are robust, and appear in all results even though a variety of surface heating functions were used, and no flow field was present. If instead of this cartesian coordinate system, along and across-isopycnal mixing is employed, the temperature structure turns out to be depth independent and resembles the isotropic limit (large $\kappa$ ) of the cartesian model. The addition of the flow field to the isotropic limit will lead back to the features described above, and will be further investigated in chapter 5 . The contrast between the two results suggest that the missing physics in the isopycnal model (like a flow field) is rather important in oceanic studies.

Two other comparisons were made between different heat fluxes. In the first we concluded that the differences between specifying the heat flux and specifying the air temperature, are small for large $\kappa$ 's. These small changes include colder fluid when the air temperature was specified, and a slight northward shift of the convective overturning region. In the second comparison, the differences between a steady state and average of periodic states was investigated. Our results carry an important message to atmosphere-ocean models using climatological forcing. In averaging periodic states, the winter events of strong vertical mixing in southern latitudes caused the surface water to warm up by about $4^{\circ} \mathrm{C}$, thus, the resulting air-sea temperature gradient will be different. Also, in this procedure one warm summer month is enough to overcome the signature at all marginally stable locations at other months, resulting in stable stratification everywhere. Based on these differences between averaging instantaneous realizations and averaging the forcing, one should always be clear about the type of 
averaging procedure used. Regardless of the averaging taken, by bringing down cold water in the north, the mixing causes the deep fluid to become colder and so the isotherms are more surface intensified in the south. 


\section{Chapter 3}

\section{Water mass formation by buoyancy loss without advection}

\section{Introduction}

In the previous chapter the concept of convective overturning was introduced and applied in regions where the vertical temperature profile was unstable. Here, the same idea is used to formulate a simple model that describes the formation process of chimneylike structures in open water. This model is fairly general and is not an attempt to explain the details of any particular deep water mass formation. It should only be understood as highlighting the possible importance of vertical mixing in the process.

The concept of water masses in oceanography can be exemplified by Wüst's Core Layer Method. In his arguments Wüst shows that if we look at tracer maps in the ocean, we find tongue-like shapes of tracer properties. It then seems reasonable to assume that the direction of the flow in that region follows the shape of the tongue. It is true that the rotation of the earth and diffusion can cause tracer distribution patterns unlike the flow pattern, [Neumann and Pierson (1966), pp. 407] but still Wüst's Core Layer Method, was and still is justifiably popular as giving an indication of the flow pattern, and pointing to possible tracer source locations. An important question in physical oceanography is to investigate the origins of these water masses, and the processes under which they were formed. 
In our simplified model we add salinity to our temperature field and let the density depend linearly on both salinity and temperature. By introducing different heat and salt fluxes at the surface, we create chimney-like structures in our fluid; in those chimneys the density and salinity are homogenous. From these chimneys (which were created by mixing only), tongues of water masses can spread horizontally by diffusion and advection.

Most of the work relating to water formation has to do with regions like the Mediterranean, Weddell, Greenland, and Labrador Seas. The few observations available support the idea that the waters are formed in chimney-like structures, for instance MEDOC Group (1970), Gordon (1978) and (1982), Carmack and Aagaard (1973), Lazier (1973) and Clarke and Gascard (1983). At those locations, water masses are formed by open water convection or along continental shelves, and can afterward be traced as deep boundary currents. Although our ideas can be applied to these regions, they can also be utilized in explaining the formation of known shallow water masses.

Evidence of "near surface" water masses are often encountered in the data. For example, the high salinity Levantine Intermediate Water in the Mediterranean Sea appears clearly in data plotted by Wüst (1961). These waters are found at a depth range of $200-600 \mathrm{~m}$. Another example is the upper portion of the high salinity water in the Eastern North Atlantic. Based on a new analysis by Pollard and Pu (1985) this water mass is not related to the Gibraltar Straits outflow, but is actually separated from the outflow region by a layer of minimum salinity, a layer that is ventilated north of the outcropping of the anticyclonic wind driven circulation. The last notable example is mode water like the $18^{\circ} \mathrm{C}$ water in the Sargasso Sea. This water mass is formed in the winter time by atmospheric cooling, and is easily identified year-round by looking at isotherms in the Eastern North Atlantic.

In his overview of mode waters at different locations throughout the world, McCartney (1982) explains that they are characterized by their homogeneity, in particular, 
a minimum vertical gradient anomaly with respect to their surroundings. In a regional volumetric census, this result will show up as a distinct $T-S$ class. Locating the tongueshaped paths of the mode water away from their convective source is done by following their minimum of potential vorticity, which is a result of their minimum stability.

Obviously, there were other modeling efforts relating to water mass formation. Notably, Killworth (1976) modeled the violent mixing stage observed in MEDOC, an experiment with which we will also compare results. Killworth model is more involved than ours, and couples the convection to geostrophic adjustment by using the full set of the equations of motions. He obviously has the advantage of getting a more detailed evolution picture of the process, and after carefully choosing the thermal diffusivity coefficients and a very special initial density profile, he is successful in duplicating various aspects of the MEDOC observations. In contrast to Killworth, our model is simpler and emphasizes the general features of the formation process, in particular, by stressing the importance of mixing in the procedure. 


\section{The model}

The chimney structure is characterized by vertical homogeneity of density and other tracer quantities, such as salt. But although the fluid inside the chimney has the same density as the fluid right at the base of the structure, (otherwise the chimney would penetrate to deeper or shallower depth) the tracer concentration inside it is different. This new tracer value results from vertical mixing of a quantity whose concentration is often depth dependent.

The generation of a chimney adds a certain volume to a certain density layer, and takes mass from the density layers above this particular density. Because this added mass has different properties than those found in the density layer, the tracer properties of the density layer change when the chimney erodes by preferred alongisopycnal advection and diffusion. This will be observed as tongue-like shapes with different tracer concentration. If the chimney is an isolated feature, the new added mass and tracers are miniscule, and the effect will quickly disperse. On the other hand, if we envision a situation with many chimneys whose formation process, albeit irregular, repeats itself in time, we can easily create a local mode water.

The formation process we propose is simple yet effective. Wherever there is buoyancy loss at the surface, say due to heat loss or loss of fresh water, vigorous vertical mixing is initiated to erode the new unstable stratification. The longer in time and the broader in space the buoyancy loss is, the deeper and larger the chimney will be. Of

course other processes could enhance the structure, in particular, local preconditioning can help a short event of buoyancy loss to contribute in converting shallow and intermediate water into a deep water mass.

Because of the simplicity of the model one can look at the buoyancy loss events as a perturbation, and impose them on some general heat flux pattern, like the Oort and Vonder Haar data used in the previous chapter. But imposing small random variations on the forcing does not lead to the water mass production described here. Not only is 
the ocean stably stratified in the upper part of the water column, but also the time scale for non-turbulent diffusive processes is large. In order to have water mass production, the perturbations-depending on preconditioning-should be strong and steady, for periods usually longer than a week. Obviously, in a long enough series of random perturbations one cannot exclude the possibility of events of water mass production, but by and large the effects of the perturbations will only graze the surface. Similar results were also derived analytically by Bryden and Stommel (1984). The authors showed that there is a critical value of buoyancy loss that must be associated with the Mistral winds in the western Mediterranean if they are to be successful in producing deep water. On the other hand, based on observation, one can justifiably argue that in reality the perturbations are very large, much larger than the mean. For example, the daily heat loss in the period of the Mistral was several times larger than before it. So if we want to call the Mistral a perturbation to the local weather pattern, we probably have to restrict it to occur not more than a few times a year. In that respect we cannot treat it as a perturbation in the regular sense of a small amplitude random event.

One of the important features of the model is the lack of circulation. This is not to argue that the velocity field is not important, but rather to show how far one can advance with diffusive processes alone.

Because the formation depends on buoyancy loss, one can generate chimneys even in simple cases where the density depends linearly on temperature alone. For instance, a regions of vertical homogeneity is observed in figure (2.3)e. But in order to observe tongue-like shapes spreading from a formation region, one needs an additional tracer, chosen here as salt. Inside the chimney the temperature and salinity are homogeneous in the vertical, but are not necessarily the same as those at the base of the chimney [see for example figures (3.2)a,c and (3.4)a,c which will be analyzed shortly]. In the erosion process, this water with different salinity will be observed as the tongues mentioned 
before. The diffusion equation for temperature and salinity, and the new relationship for the density are written as

$$
\begin{aligned}
T_{t} & =T_{y y}+\left(\kappa_{z} T_{z}\right)_{z} \\
S_{t} & =S_{y y}+\left(\kappa_{s} S_{z}\right)_{z} \\
\rho & =\rho_{0}\left[1-\alpha\left(T-T_{0}\right)+\beta\left(S-S_{0}\right)\right]
\end{aligned}
$$

where $\kappa_{T}$ and $\kappa_{s}$ are the dimensionless diffusion coefficients for temperature and salinity. They are not necessarily the same, and their relative size is less important in the generation process as long as they become large enough when the stratification is unstable.

$$
\kappa_{s}, \quad \kappa_{T}= \begin{cases}O(1), & \rho_{z} \geq 0 \\ \rightarrow \infty, & \rho_{z}<0\end{cases}
$$

\section{Numerical implementation}

The numerical scheme for solving this set is identical to the previous implementation in chapter 2 , and involves adding time derivative terms $T_{t}$ and $S_{t}$. The notable difference between the two is that in solving (2.1) we look for steady state solutions, where $T_{t}=0$. Here, a water mass formation process is described, so the problem is inherently time dependent, and a steady state is not reached. In all cases, the final states describe the fluid after running the model for the same amount of real time. Both the initial condition (stratification) and boundary condition (imposed buoyancy loss) have a pronounced signature on the generated structure. The grid resolution in the horizontal and vertical is $16 \times 64$, and $\kappa=1000$. 


\section{Results}

The results of the water mass formation process model are exhibited in figures (3.1)(3.4). These were obtained using the set (3.1), by time stepping the diffusion equations for temperature and salinity, and increasing the vertical mixing coefficient when the density was unstable. In order to isolate the effects of winter cooling, we specified zero salt flux through all boundaries at all times, and zero heat flux except for a short step function along the upper boundary. This is equivalent to confining the fluid into an insulated box, with a small hole in its upper wall through which we cool the system, and sits well with the idea of looking at the process as a strong event superimposed on some annual cycle. The localized nature of the event will be further commented on later in this chapter. Because of the linearity of the equation of state, similar results can be obtained by replacing the heat flux with loss of fresh water, or some combination of the two. Thus, one can apply the same process to describe phenomena in regions where intense cooling is more dominant, $\left(18^{\circ} \mathrm{C}\right.$ mode water $)$ or where loss of fresh water is caused by warm summers (Eastern Mediterranean regions).

We examine the effects of two parameters on the process: the initial stratification (which is always stable), and the strength of the heat flux. Figures (3.1)-(3.2) represent the cases with an initial linear temperature profile in the vertical $(T \propto z)$ and a exponential salinity layering $\left(S \propto e^{3 z}\right)$. The other figures (3.3)-(3.4), correspond to a surface intensified exponential temperature and salinity profiles $\left(T, S \propto e^{4 z}\right)$. It is not of paramount importance that the salinity decrease with depth as rapidly as the temperature. As mentioned before, it has no effect on the possibility of chimney creation. In our example, it is simply more instructive. We start with a strong surface signature of salinity, and show that after generating the well mixed region in the vertical, a new salinity maximum corresponding to a density different than that in the top layer can be found. The effect is more pronounced if the initial salinity variations are strongly surface intensified, as in the case of the exponential profiles chosen here. 

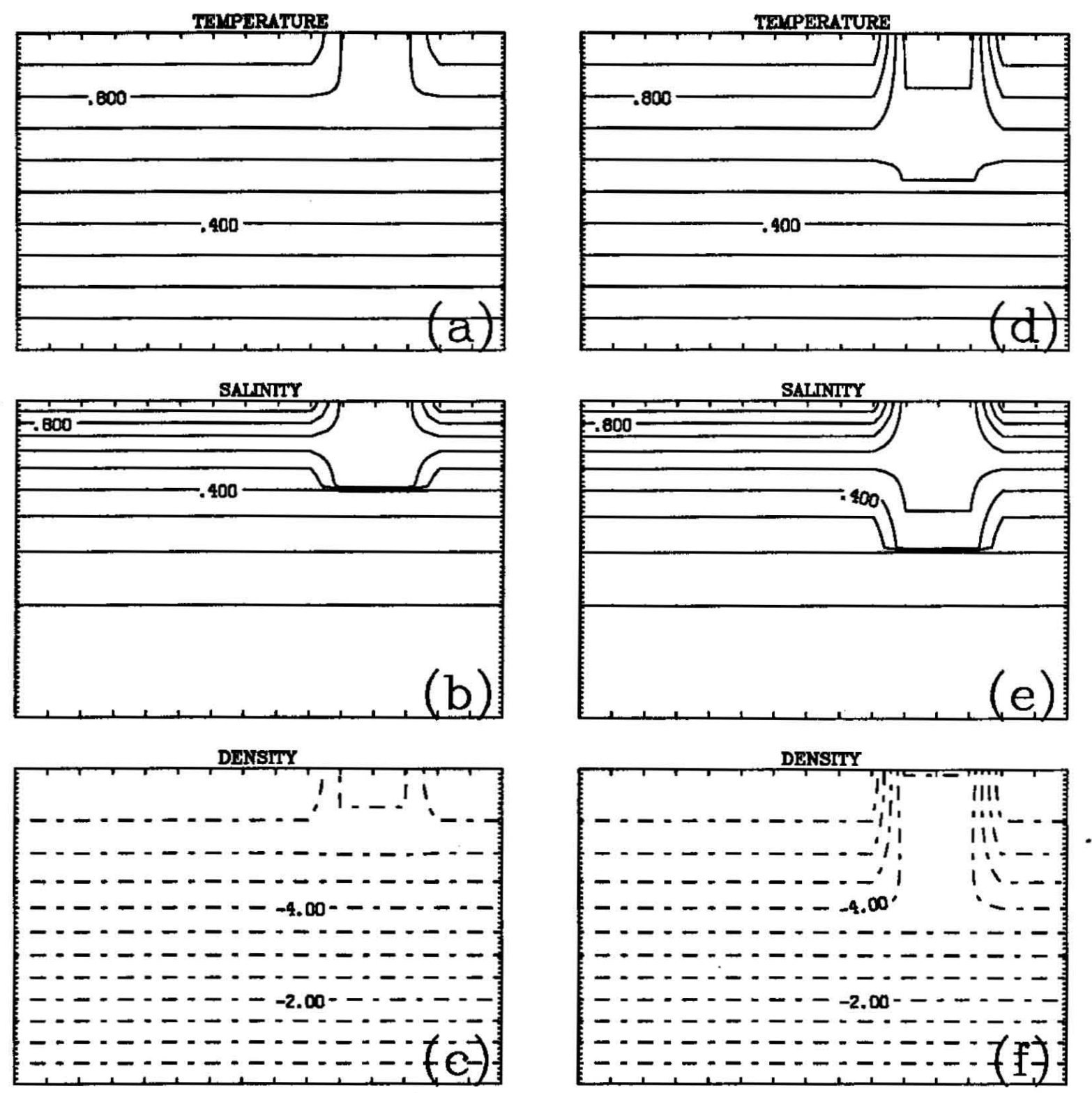

Figure (3.1). Chimney generation when the initial stratification is $T \propto z$, and $S \propto e^{3 z}$. The specified cooling at the top is one unit for the left column, and four units for the right. (a), (d) Temperature. (b), (e) Salinity. (c), (f) Density. 

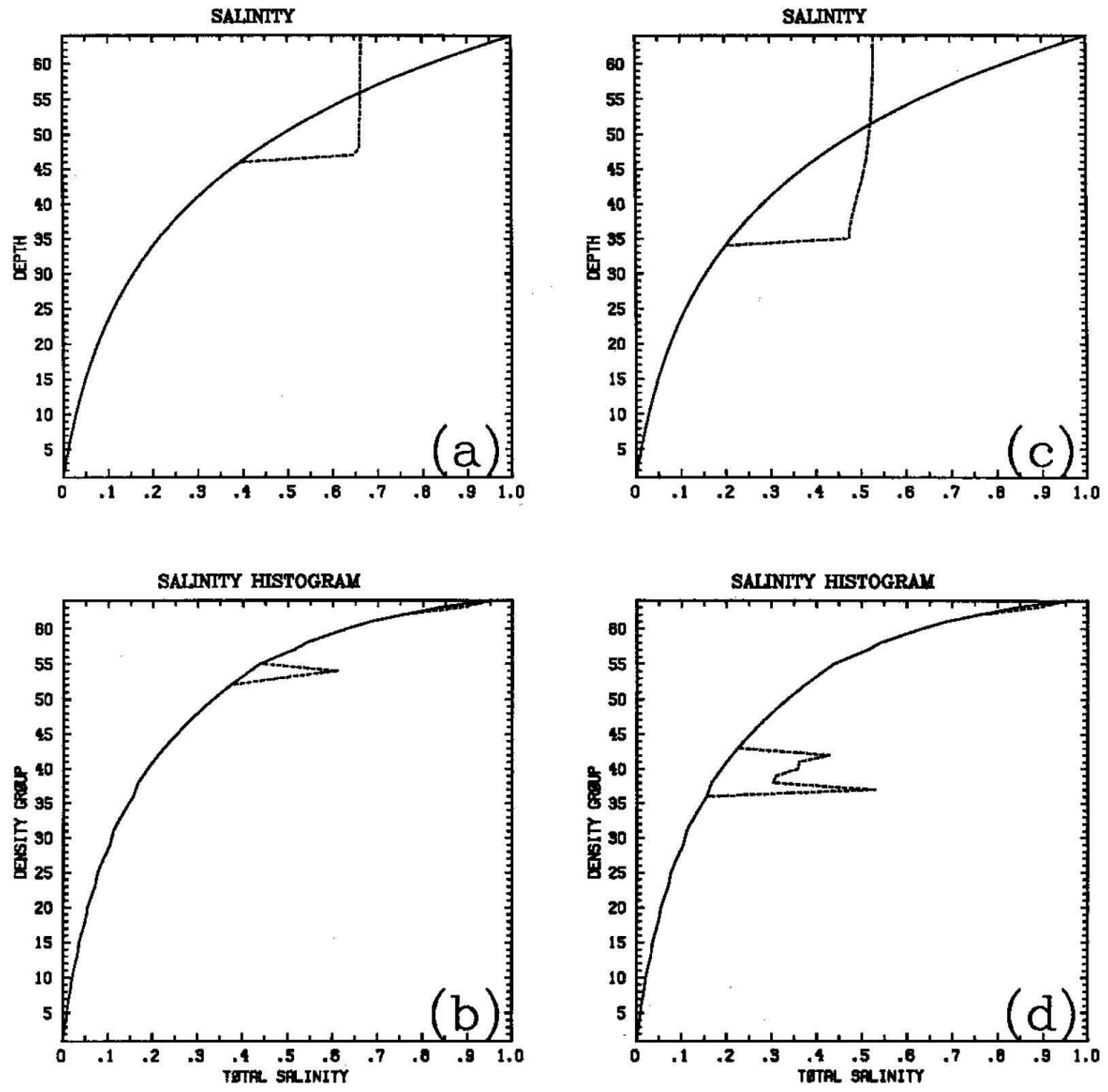

Figure (3.2). Information about the salt content for the conditions of figure (3.1). The left column represents a unit heat flux while the right column is four times the cooling. (a), (c) Variations of salinity with depth. (b), (d) Salt content in each density layer weighted by the volume of that layer. In both, the solid line is the situation before the formation of the chimney, the dashed line, after the chimney's generation. 

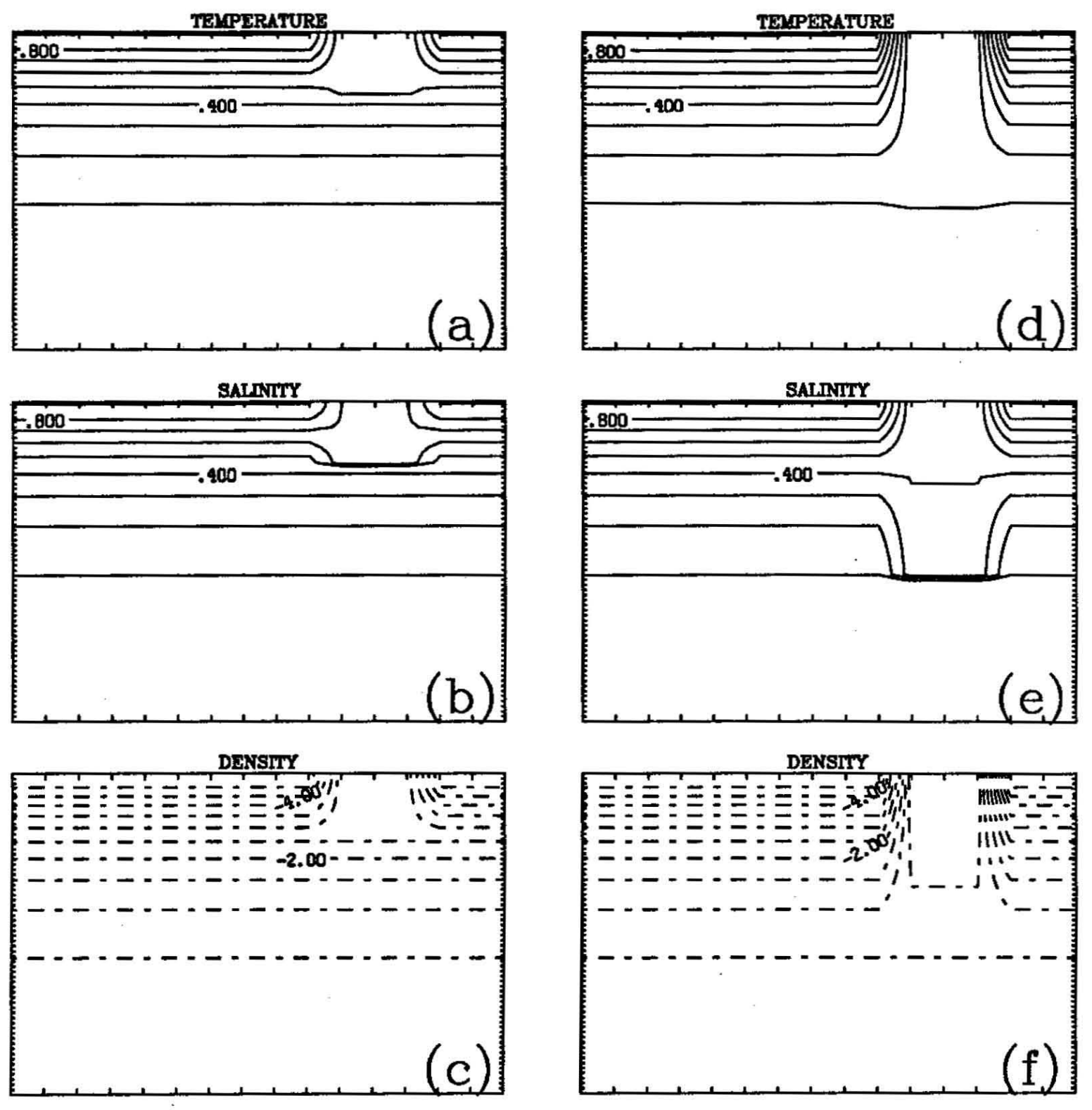

Figure (3.3). Same as (3.1) but $T$ and $S$ are $\propto e^{4 z}$. 

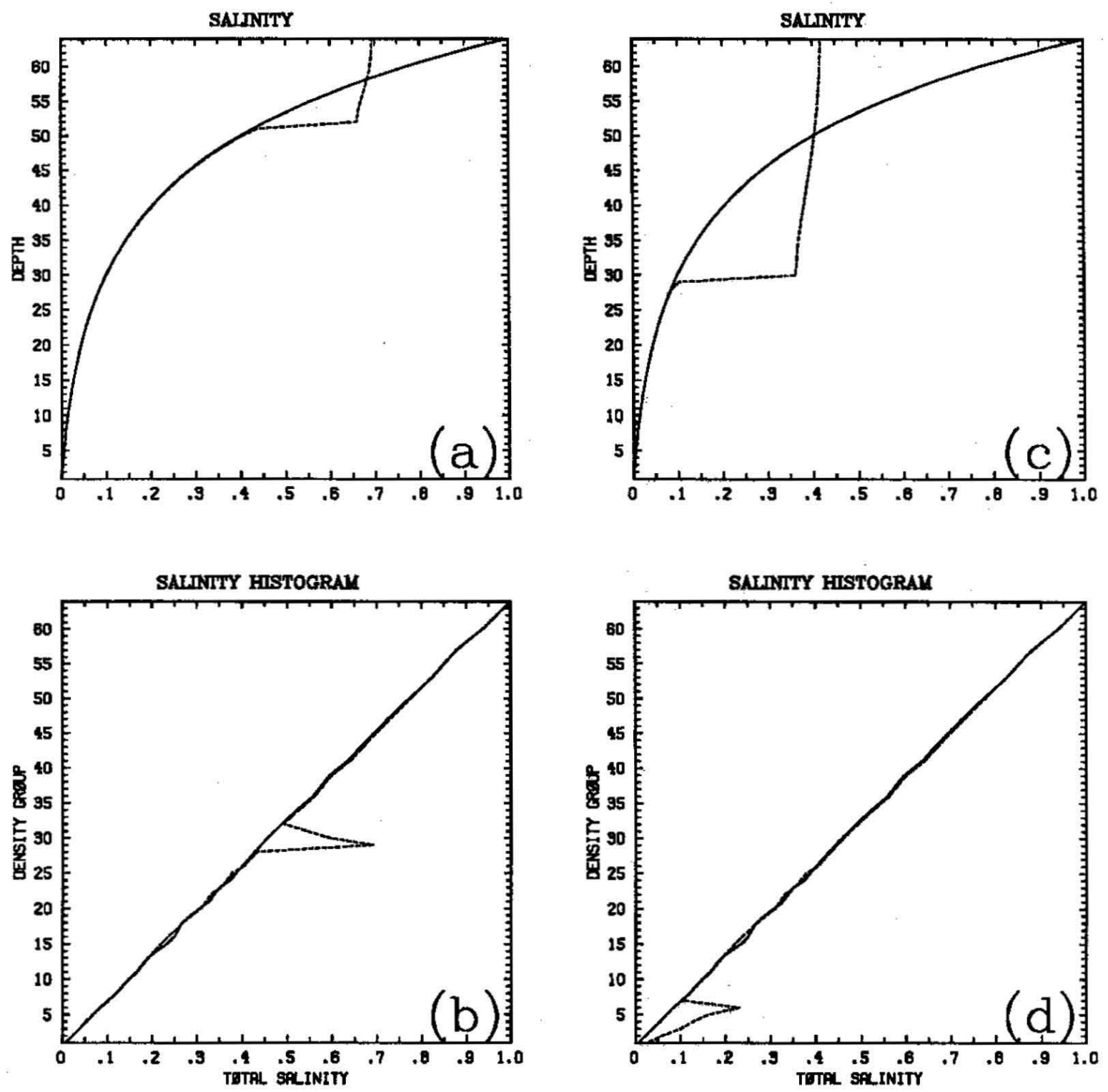

Figure (3.4). Same as (3.2) but for the conditions presented in figure (3.3). 
Two heat fluxes are examined. The units are irrelevant as long as we note that one is four times as large as the other. Thus, figures (3.1) a-c, (3.2) $a-b,(3.3) a-c$, and (3.4) $a-b$ are with a heat flux of 1 , while (3.1) $d-f,(3.2) c-d,(3.3) d-f$, and (3.4)c-d are with a heat flux of 4 . Overall, there are two different initial stratifications and two distinct heat fluxes, leading to the four possible combinations summarized in table (3.1).

\begin{tabular}{|c|r|r|r|}
\hline Figure & $\begin{array}{c}\text { Surface Heat } \\
\text { Flux }\end{array}$ & $\begin{array}{c}\text { initial temperature } \\
\text { structure }\end{array}$ & $\begin{array}{c}\text { initial salt } \\
\text { structure }\end{array}$ \\
\hline$(3.1) \mathrm{a}-\mathrm{c},(3.2) \mathrm{a}-\mathrm{b}$ & 1 & $z$ & $\frac{e^{3 z}-1}{e^{3}-1}$ \\
$(3.1) \mathrm{d}-\mathrm{f},(3.2) \mathrm{c}-\mathrm{d}$ & 4 & $z$ & $\frac{e^{3 z}-1}{e^{3}-1}$ \\
$(3.3) \mathrm{a}-\mathrm{c},(3.4) \mathrm{a}-\mathrm{b}$ & 1 & $\frac{e^{4 z}-1}{e^{4}-1}$ & $\frac{e^{4 z}-1}{e^{4}-1}$ \\
$(3.3) \mathrm{d}-\mathrm{f},(3.4) \mathrm{c}-\mathrm{d}$ & 4 & $\frac{e^{4 z}-1}{e^{4}-1}$ & $\frac{e^{4 z}-1}{e^{4}-1}$ \\
\hline
\end{tabular}

Table (3.1)

For each of these four cases we present five figures: temperature, salinity, density, variations of salinity with depth before and after the generation of the chimney, and salinity histograms (amount of salt in each density layer weighted by the volume of that layer) before and after the process. The temperature, salinity, and density [figures (3.1) and (3.3)] show that changes from the initial stratification outside the chimney region are very small. This is a result of our choice of weak horizontal diffusion and intense enough cooling so as to create the chimney in a short time span. In addition, the figures clearly show that the fluid inside the chimney is very well mixed, with almost constant salinity, temperature, and density.

These results are further confirmed by looking at the variations of salinity with depth before and after the chimney's creation [figures (3.2)a,c and (3.4)a,c]. The solid line in each figure represents the situation before the generation, and the dashed line that after the generation. The two lines overlap at all depths larger than the chimney depth, where they separate with an abrupt jump. From there to the surface, the dashed 
line is nearly vertical, representing the well-mixed salinity in the chimney. The size of the salinity jump at the base of the chimney indicates how much larger the salinity in the chimney is compared to that in the density layer below it. Once the chimney erodes, its fluid with relative high salinity will mix with that in the appropriate density layer, thereby generating the high salinity maximum.

The histograms in figures (3.2)b,d and (3.4)b,d represent the total amount of salt in each density layer divided by the volume of that particular density layer

$$
S^{T}\left(\rho^{\prime}\right)=\frac{\int S\left(\rho^{\prime}\right) d x d y}{\int \delta\left(\rho, \rho^{\prime}\right) d x d y}
$$

where the integration is taken over the whole basin, $\rho^{\prime}$ is a particular density, and $\delta$ denotes the Dirac delta function. The solid line in each of the figures stands for the salt content before the generation of the chimney, while the dashed line is the salt content afterwards. To a good approximation, the two lines overlap with the exception of the dashed peak. Consider, for example, a density layer that lies above the base of the chimney. Before the generation of the chimney, it had a certain salinity (defined as the total amount of salt it that density layer divided by the volume of the layer). After the formation event, both the volume and the total salt content of the layer decreased (because a certain amount of fluid was removed form that layer), but the salinity of the layer is not changed. The only layer whose volume and total amount of salt changed in a way as to affect the salinity, is the layer at the base of the chimney. The amount of water type of that density increased by the volume of the chimney, but the salinity of the chimney is not the same as that in the density layer under it. Thus, we get a new peak below the surface showing that the salt content of this density layer increased. The size of the peak depends on the relative size of the chimney with respect to the basin and the initial amount of fluid in the density layer corresponding to the chimney's density. All other things being equal, if we were to make the chimney wider, the peak would be bigger. 
Because the salinity in the chimney is higher than at the corresponding density layer [note the jump in figures (3.2)a,c, and (3.4)a,c], once the chimney collapses by along-isopycnal processes, it will be possible to observe a tongue shape of salinity maximum spreading from the formation region.

The relative contribution of salt and temperature to the density field can easily be assessed from the graphs. In figures (3.1)-(3.2) the temperature is linear with depth while the salinity is exponential, and the resulting density field is somewhat bottom intensified. In general, as the equation of state (3.1c) shows, the two fields tend to counteract each others contributions to the density. Higher temperature causes the fluid to become less dense, while higher salinity causes density to increase. The net contribution of these two opposing effects usually favors the temperature because even though as a rough order of magnitude $\beta \approx 4 \alpha$, the temperature variations can easily be 20 times those of salinity, so the net effect is that the temperature variations dominate. Moreover, even though for small scale processes like salt fingers, the differences between the molecular diffusivities of salt and heat are important, It is not clear that the eddy diffusivities are not approximately equal. In that case, one only needs one parameter in the equation of state, which is why one often uses equation (1.1f) instead of (3.1c).

Comparing figures (3.1) a-c to (3.1) d-f, (3.2)a-b to (3.2)c-d, (3.3) a-c to (3.3) $d-f$, and (3.4) $a-b$ to (3.4) $c-d$, we note that when the cooling is increased by a factor of four, the chimney does not penetrate to a depth four times greater, but only to about twice the depth when the temperature layering is linear, and less than three times the depth when it is exponential. The proper analytic expression will be derived shortly. 


\section{Discussion}

One of the purposes of the process model is to show that one can generate a source for a water mass by a mixing process. This idea is especially important if we remember that in all other processes where a flow field is present, some sort of vertical circulation is implied. This vertical circulation is not observed in many locations where shallow water masses are found. In addition, conservation of mass requires that if there is downwelling in the water formation region, there would be upwelling somewhere else. This upwelling next to mode water locations is not reported.

In our pictures, the water mass is distinguished by its salinity maximum; this of course is only an example, and many other tracers would be good candidates, including those that do not contribute directly to the equation of state.

In all our examples the salinity in the well-mixed chimney corresponds to the salinity in a density layer that lies at about half the chimney's depth. The exact location is the intersection between the dashed and the solid lines in figures (3.2)a,c and (3.4)a,c, each for a different initial layering and heat flux. When the formation process stops, this pool of saline water will advect and diffuse along the density surface corresponding to the density inside the chimney. This density surface obviously lies at the base of the chimney. This mixing process increases the amount of salt in that particular density layer. The salinity histograms (3.2)b,d and (3.4)b,d show the new maximum clearly. It is this salinity (or other tracer) maximum that is found in available data at different locations throughout the world oceans.

The above argument relies heavily on the idea that once a chimney is created, diffusion and advection tend to occur along isopycnal surfaces rather than across them, because these surfaces represent geopotential surfaces. If this is indeed the case, the new pool of water created will join the appropriate density layer at the bottom of the chimney, thereby changing its average tracer properties. 
The erosion of the chimney can be examined in several ways. For instance, one can rewrite the equations in density coordinates by replacing the vertical coordinate $z$ with $\rho$. Alternatively, we could keep the present coordinate system, but replace the diffusion coefficient in the heat equation and salt equations (3.1)a-b by the second degree tensor introduced in (2.3). Any one of these schemes can be used to further describe the salinity propagation after we established its source as the chimney.

Depending on the initial stratification and the strength and duration of the fluxes imposed at the top, the chimneys can reach different depths. This depth is important because it represents the depth of the density layer along which fluid created in the well-mixed chimney moves. To compute the depth $-h$ of the chimney given a total amount of heat $\mathcal{K}^{T}$, we introduce to the following notations. Let $\rho(\bar{S}, \bar{T})$ be the density in the chimney, $\rho(S(-h), T(-h))$ the density at the base of the chimney, and $\bar{S}=$ $\frac{1}{h} \int_{-h}^{0} S(z) d z$ the salinity in the chimney. Because $\rho(\bar{S}, \bar{T})=\rho(S(-h), T(-h))$ we must have

$$
\bar{T}=T(-h)-\frac{\beta}{\alpha}\left[S(-h)-\frac{1}{h} \int_{-h}^{0} S(z) d z\right] .
$$

The total amount of heat is thus

$$
\begin{aligned}
H^{T} & =\int_{-h}^{0}\left[T(z)-\bar{T}-\frac{\beta}{\alpha}(S-\bar{S})\right] d z \\
& =\int_{-h}^{0}[T(z)-T(-h)] d z-\frac{\beta}{\alpha} \int_{-h}^{0}[S-S(-h)] d z .
\end{aligned}
$$

Because $T(z)$, and $S(z)$ are known, $h$ can be computed given $\mathcal{H}^{T}$, as presented in figure (3.5). When starting with a linear temperature stratification, $T=z,(3.2)$ gives $z_{m} \propto \sqrt{K^{T}}$ if $\beta=0$ (no salt), which is the reason our computations are done for two heat fluxes differing by a factor of four. For this linear case, increasing the total amount of cooling by four causes the chimney to penetrate to twice the depth. The result is confirmed by comparing figures (3.1)a-c to (3.1)d-f. The second, with four times the heat flux, shows a chimney about twice as deep. The exact numbers from figures 
(3.2)a,c show a depth gain of $30 / 18 \approx 1.7$. The value is smaller than 2 , because as the second term in (3.2) indicate, and as we know from the equation of state (3.1c), the salinity has a destabilizing effect on the density. As we penetrate deeper, the salinity decreases, thereby making the fluid lighter, hence part of the cooling is compensating for that effect and not acting to make the chimney deeper. As seen by comparing figures (3.3)a-c to (3.3) $\mathrm{d}-\mathrm{f}$, for an initial exponential layering the increase in depth is more dramatic. The exact depth gain in this case is $35 / 13 \approx 2.7$, and is based on figures (3.4)a,c. Nonetheless, small changes in the heat flux will not cause significant changes in the depth of the salinity maximum. Even if we look at a very cold winter with $50 \%$ more cooling, the penetration depth will increase only to $360 \mathrm{~m}$ from an initial $300 \mathrm{~m}$, assuming linear temperature profile and no salt. This relative insensitivity of depth to climatic variations is encouraging, and means that the vertical location of the center of the water mass formed will be fairly stable.

Similar conclusions were also obtained by Warren (1972) by considering that the energy budget in a water column consists of a balance between the absorbed short wave solar radiation, the net long wave radiation emitted from the sea, and the sensible and evaporative heat fluxes. The empirical forms for these constituents were approximated by Newtonian cooling law. It is important to note that Warren assumes that the temperature structure in the thermocline is linear with depth, which leads him to the same power law we obtained here for the similar case.

Solutions to (3.2) for the cases investigated are presented in figure (3.5) as a $z$ versus total heat graphs. The value of $\beta / \alpha$ is taken from data from the Eastern Mediterranean presented in figure (3.6), where $\Delta T=22^{\circ}-14^{\circ}=8^{\circ} \mathrm{C}, \Delta S=39.1 \%$ $38.6 \%=0.5 \%$, and $\beta$ is four times larger than $\alpha$. For these values the dimensionless $\frac{\beta}{\alpha}=\frac{\beta^{*}}{\alpha^{*}} \frac{\Delta S}{\Delta T}=0.25$, with asterisks denoting dimensional quantities. The dashed line in the figure corresponds to the exponential temperature and salinity [figures (3.3)(3.4)], and the solid one to the linear temperature and exponential salinity [figures 


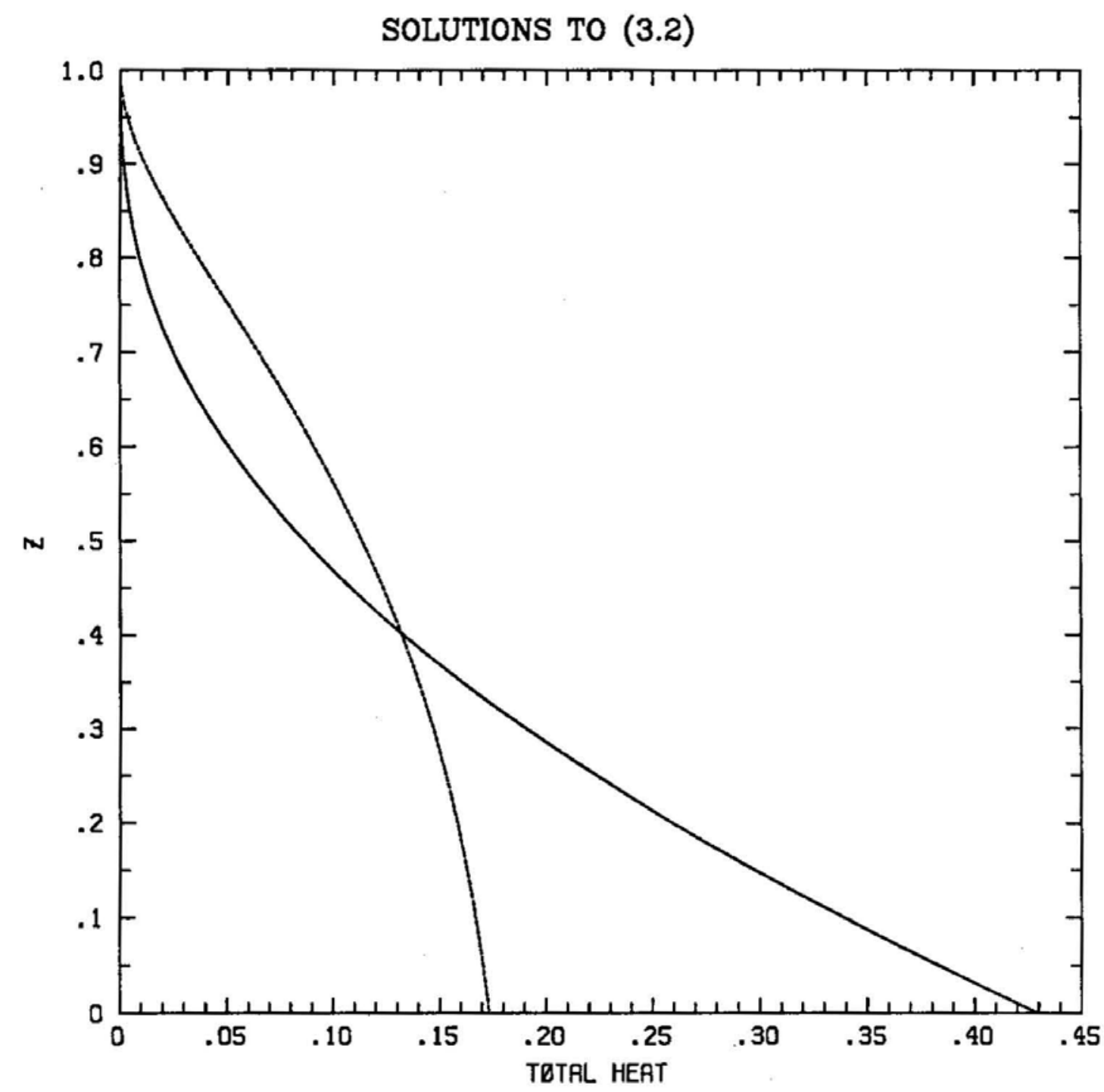

Figure (3.5). Solution to equation (3.2) for the two cases investigated in the text. The solid line corresponds to $T \propto z$ and $S \propto e^{3 z}$, the dashed line to $T$ and $S \propto e^{4 z}$. 
(3.1)-(3.2)]. One can clearly see that a much smaller amount of heat is to be removed if we want to reach the bottom in the doubly exponential case. Also, when the depth is large enough-say, below the intersection of the two lines-a small amount of cooling would cause a pronounced depth change when both fields are exponential in $z$. Similar graphs for the same $T$ and $S$ profiles but for different dimensionless $\beta / \alpha$ will have the same shape but slightly removed to the left or right of the current lines. Because the salinity is destabilizing the stratification, curves with smaller $\beta / \alpha$ will be to the right, while the others will move to the left. When both $T$ and $S$ are exponential in $z$, the intersections of the dashed curve with the $z=0$ line are at a total heat values of $(0.197$, $0.174,0.150)$, when $\beta / \alpha=(0.15,0.25,0.35)$ correspondingly (the middle value is the one used in this section). This indicates that even large salinity fluctuations have small effects on the profile, and although it is true that smaller amount of heat is needed to reach the same depth when there is more salt, the similarity between the different curves suggest that one cannot altogether neglect the local variation of the profiles with depth if one wants to account for small changes.

Not much is known today about the dynamics of water mass formation, and it is not the purpose of this work to elaborate on these issues. For example, in the model proposed here, the formation event is realized by localized buoyancy loss, and questions like why one location is preferred over another, how many chimneys are there, etc. are not treated. But it is important to note that in the ocean, one would tend to assume that forcing is not localized, hence the buoyancy loss is much broader than the region of the chimney. As reported by Stommel (1972), the buoyancy loss in the upper 1000 meters due to the Mistral in the MEDOC experiment was uniform over a region spanning 200 kilometers, which is much larger than the reported $50 \mathrm{~km}$ width of the chimney. Stommel noted that before the Mistral, the buoyancy in that region had a bowl shape (concave) so when the additional uniform buoyancy loss due to the Mistral is superimposed on that shape, the result is a narrow region whose buoyancy is reduced 
to zero, corresponding to the diameter of the observed chimney. Stommel concludes that the stratification before the event is very important in determining the site of the chimney. One can also hypothesis that once a chimney is generated, there is a 'drain' in the region, so if the buoyancy loss is increased, the chimney does not necessarily gets wider, but perhaps there is some surface flow into the chimney's region.

\section{Relation to observations}

Unfortunately, the available data to date on water formation processes is very limited, due mainly to the irregularity of the process in both time and space, the narrowness of the formation region, and the less than ideal data gathering conditions. On the other hand, water masses of distinct properties are commonly observed even if their formation process can only be speculated upon. In the examples below we relate our model to some of the available measurements.

Observations in the Eastern Levantine basin in the Mediterranean Sea, for example, Wüst(1961), show a persistent salinity maximum at depth of about $300 \mathrm{~m}$. A typical salinity profile [Hecht (private communication)] taken at $33.00^{\circ} \mathrm{N}, 33.50^{\circ} \mathrm{E}$ is represented in figure (3.6)b.

The available data indicate that this feature is present all year round, and does not vary considerably in depth. This water is usually referred to as Levantine Intermediate Water (LIW). It is believed to form over portions of the Levantine basin, in particular off the island of Rhodes. Lacombe and Tchernia (1960), and Wüst (1961) reported conditions appropriate for the LIW formation north and south of Rhodes. Morcos (1972) had observations in the southern Levantine basin, whereas Moskalenko and Ovchinnikov (1965), Özturgut (1976), and Özsoy et al. (1981) consider the northeastern Levantine basin as a region of LIW formation. Preconditioning states like the one observed by the MEDOC Group (1970) were also reported by Said (1986). However, 

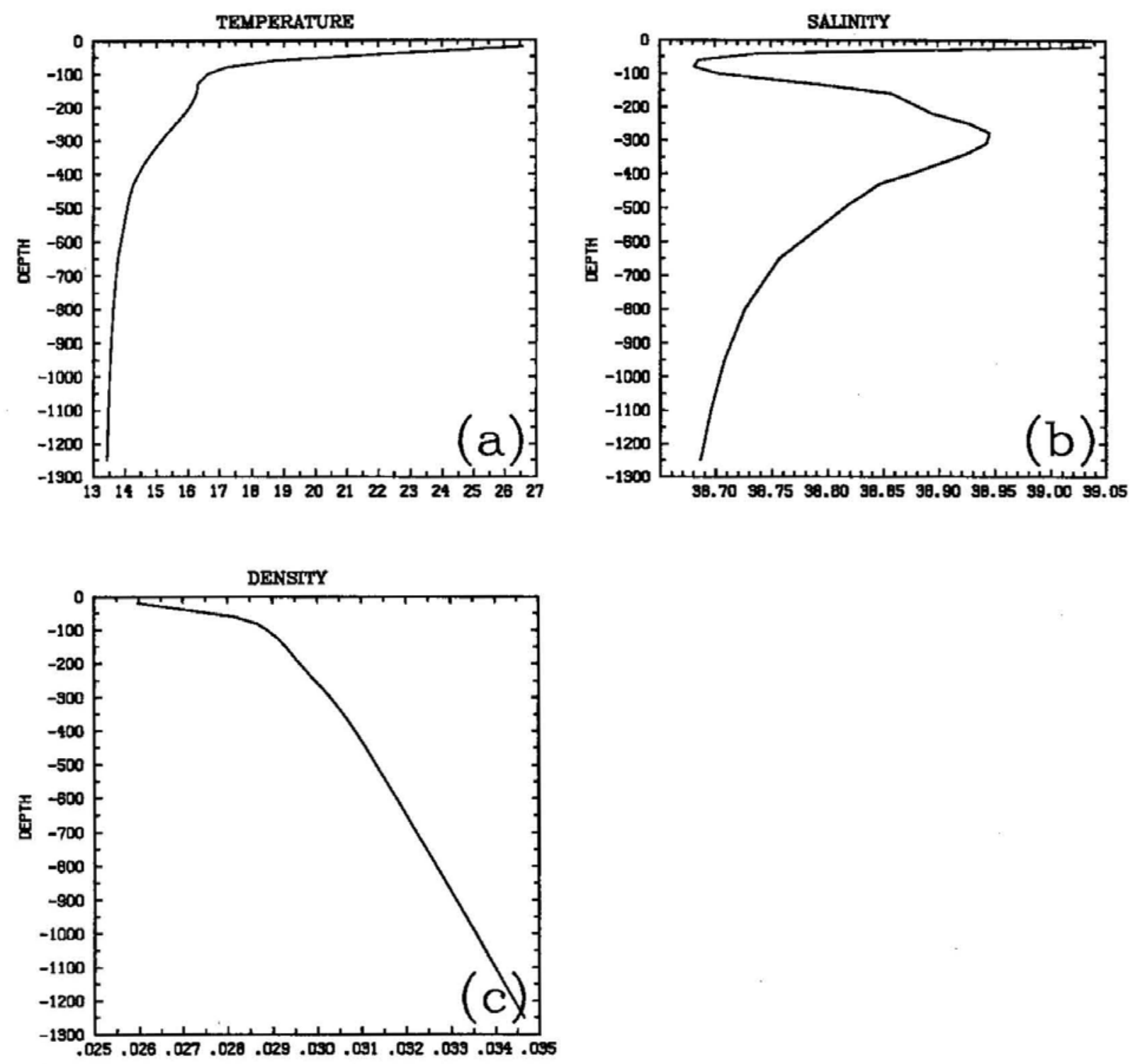

Figure (3.6). Profiles taken at $33.00^{\circ} \mathrm{N}, 33.50^{\circ} \mathrm{E}$ in the Eastern Mediterranean. (a) Temperature. (b) Salinity. (c) Density. 
one should note that direct measurements of the formation process itself, similar to the one reported by the MEDOC Group, is not available.

Although our suggested process cannot yet be confirmed by data, it is actually supported by the lack of it. At no place in the Levantine basin does one find evidence of upwelling that is necessary to compensate for sinking of LIW-if this were the main formation mechanism. Now we can explain the existence of the LIW by irregular formation of chimneys over a large portion of the Eastern Levantine basin, followed by along-isopycnal convection and advection. A similar process can account for the high salinity water in the Eastern Atlantic right on top of the high salinity outflow from the Gibraltar Straits.

The most successful observational effort to date relating to water formation was done in the winter of 1969 in the Gulf of Lyon in the Western Mediterranean Sea. These efforts were published by the MEDOC Group (1970), Anati and Stommel (1970), and Stommel (1972). The formation process was detected, and a dense observation scheme was set. The charts are published in the second article mentioned above, and show a clear chimney structure in salinity, temperature, and density-much like our results in figures (3.1) and (3.3). For instance, section 10 of their observations [reproduced here as figure (3.7)] shows a clear salinity value of $38.45 \%$ at about $900 \mathrm{~m}$, the same salinity value is observed at $400 \mathrm{~m}$. The potential temperature has the same structure as salinity, while the density contours indicate marginal stability over the full $1000 \mathrm{~m}$ of the chimney's depth.

Anati and Stommel also pointed out to the importance of vertical mixing in the process. They looked at the time variations of the vertical integral of salt and heat in the chimney. If only vertical mixing was involved, both integrals would be fixed in time, except for the heat loss by surface cooling [like figures (3.2) and (3.4)]. They found out that the integral of salt was fixed, but that of heat decreased with time. To settle the discrepancy, the authors showed that a combination of very weak surface flow 


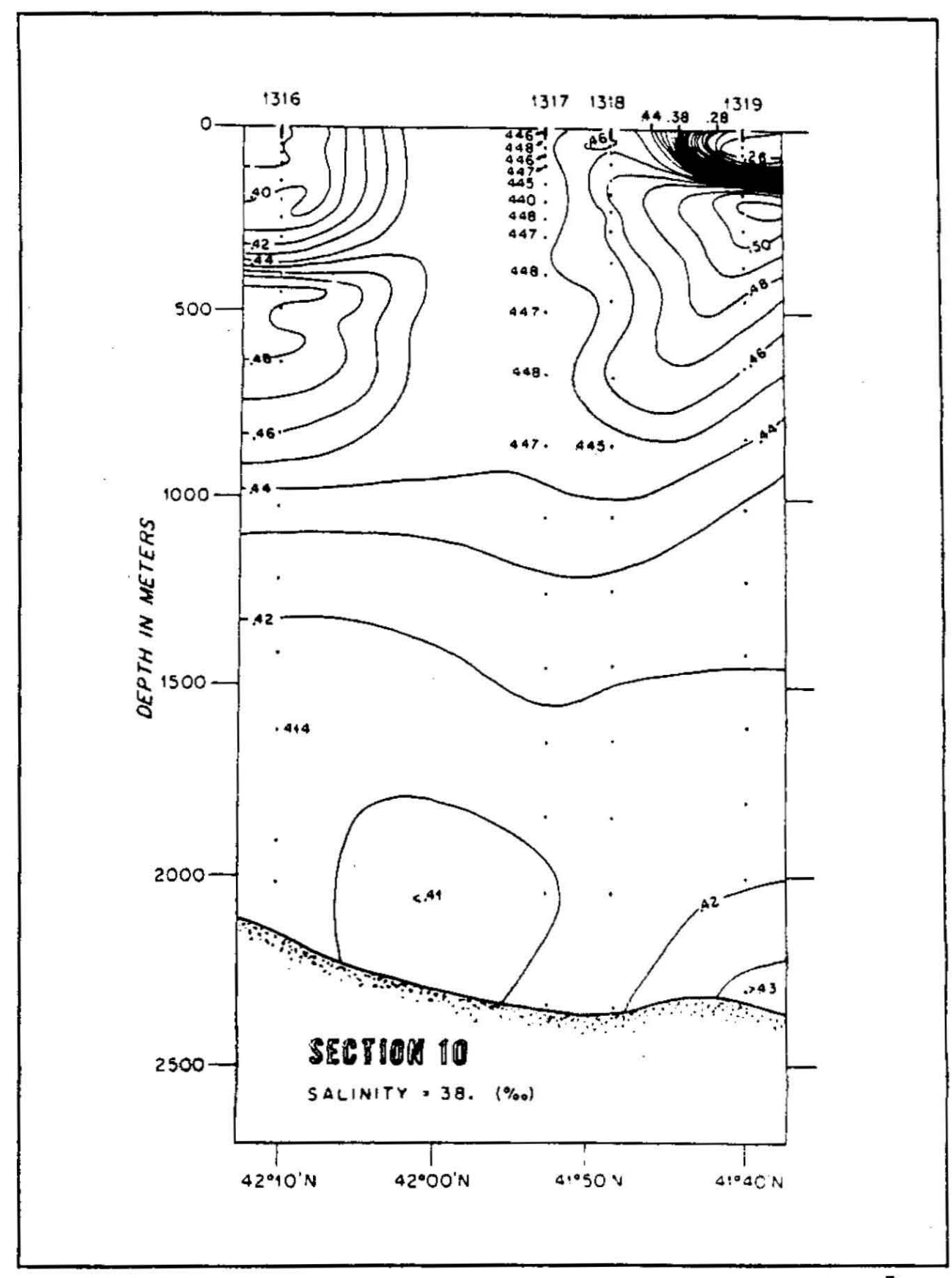

fig.na

Figure (3.7). Reproduction of section 10 of the MEDOC experiment. From Anati and Stommel, (1970). 
of fresh water from the north, and evaporation, would account for the changes in heat content, leaving the salinity practically untouched. The importance of vertical mixing in the water mass formation is one of the conclusions from that result.

The linear equation of state used in the discussion is a very good approximation in the Eastern Mediterranean, where typical values for salinity are between 38.6 and 39.1 parts per thousand, and temperature varies from $14^{\circ} \mathrm{C}$ to $22^{\circ} \mathrm{C}$. For these units, the salinity expansion coefficient $\beta$ is about four times larger than the thermal expansion coefficient $\alpha$. Because the overall temperature variations are about 16 times larger than those of salinity, it is the overall temperature structure and heat flux that largely determine the stratification. This is seen in the density profile in figure (3.6)c [taken at the same location as the salinity profile in figure (3.6) b], which barely shows the signature of the robust salinity maximum. The temperature profile in (3.6)a looks much more like the density structure than the salinity does, and is another indicator that temperature variation are important in affecting the local density. 


\section{Conclusions}

The convective overturning process was utilized to generate a chimney-like structure in the open water at locations where there is a net buoyancy loss at the surface. This pool of homogenous fluid eventually mixes into a certain density layer while modifying its tracer properties. The salinity maximum observed in the LIW, as well as other mode water found around the world's ocean, can be explained as a result of strong ver-

tical mixing (with no vertical circulation), followed by diffusion and propagation along isopycnals. The air-sea interactions which were neglected here in order to investigate the thermal forcing alone might also tend to enhance our result by strong mechanical mixing due to momentum fluxes, and by contributing to preconditioning the region. We also showed that knowledge of climatological forcing in the mode water area can lead to a good estimate of their depth. This can be used to help determining whether mixing was dominant in their formation process.

The remarkable similarity between the MEDOC Group figures and our model clearly indicate that strong vertical mixing plays an important role in the process, and represents an alternative to the traditional viewpoint which emphasizes sinking per se. 


\section{Chapter 4}

\section{Two region vertical circulation driven by cooling}

\section{Introduction}

One of most striking features of the vertical gyre is the strong asymmetric circulation forced by simple differential heating, with broad upwelling region and narrow downwelling. Because of its smallness, the northern sinking region can be looked upon as some sort of a bottle neck, which makes it important to understand the dynamics there. In the following pages, we propose a simple model where cooling from the top and the existence of a northern boundary help in maintaining a vertical gyre in a rotating fluid. Our results concerning the contributions of different dynamical processes to the circulation serves as a framework and increases our knowledge of the physics in the northern part of the ocean.

As is done in many circulation models, we simplify the problem by envisioning that the northern sinking region is composed in the vertical of a mixed layer-where the cold water temperature is approximately independent of depth-laying on top of an interior fluid. Although a model like that can be extended from equator to pole to include the broad region where the fluid returns to the mixed layer from the interior, we found it beneficial to concentrate on the particular region where fluid is being pumped from the mixed layer and not into it. This is why we often refer to it as the northern or sinking region. The restriction to northern latitudes has the obvious disadvantage 
that one might need to specify the boundary conditions along the latitude where the sinking region is connected to the rest of the vertical gyre, even though there is no rigid wall at that location. In particular, difficulties can arise from the feedback between the different boundary conditions. When the air turns colder the surface fluid will be heavier and will sink faster, thus, affecting the interior circulation by bringing colder deep water next to the surface. This will affect the conditions along the previously mentioned southern latitude, as well as the heat flux that it is proportional to the air to sea surface temperature difference.

The problem is also very interesting from a fluid dynamics point of view. The analysis of the system will lead to some remarkable results relating the conservation of angular momentum to potential vorticity, the effects of weak friction on the circulation, and the distinction between the direction of the flow field and that of the information passed in the fluid. In this chapter we will describe this two region rotating fluid model, and present some analytic solutions for the flow and temperature fields. 


\section{The model}

We envision the fluid to be confined to the geometric configuration represented in figure (4.1). Schematically, there are two regions in the vertical [denoted by (i) and (ii)], differing in their dynamics. The sense of the circulation is clockwise, so that fluid enters the upper region (i) along $y_{s}$. Due to air-sea interaction it becomes colder while moving northward, so it sinks and enters region (ii) where it completes its turn and joins the interior circulation. The geometry is constrained by a wall at the northern boundary $\left(y_{n}\right)$, and a flat bottom at $z=-D$. The interface between the two regions $h(y)$ would usually deepen as we advance northward, (possibly reaching the bottom) to account for the fact that both the downward flow field and the cooling tends to push it toward the bottom. This is also suggested by the shape of the interface in the figures presents in chapters 2 and 5.

The interface is located at depth $z=-h(y)$ and its shape can either be specified or left as a parameter. In all but one of the analytic solutions we present, we let it be a function of latitude; in the one where we do not, we assume it is flat in order to solve an elliptic equation in a convenient rectangular domain. Later it will become evident that the character of the solution is independent of the shape of the interface. Moreover, the formalism employed here can be easily extended to include the warming regions where fluid enters the mixed layer from below. This enhancement will be presented shortly.

The proper physical boundary conditions required in completing the description should include information on the temperature $\left(T_{s}\right)$ and velocity $\left(\psi_{s}\right)$ of the incoming flow, as well as the air temperature $\left(T^{\text {air }}\right)$. Information on the outgoing flow ( $\psi_{s}^{(i i)}$ and $T_{\mathrm{s}}^{(\mathrm{ii})}$ ) should probably be determined as part of the solution. The other conditions satisfied by the proposed configuration are:

(1) The temperature along the northern wall must equal that of the air. As we get very close to the wall $v \rightarrow 0$; therefore, it takes a parcel of fluid an infinite amount 


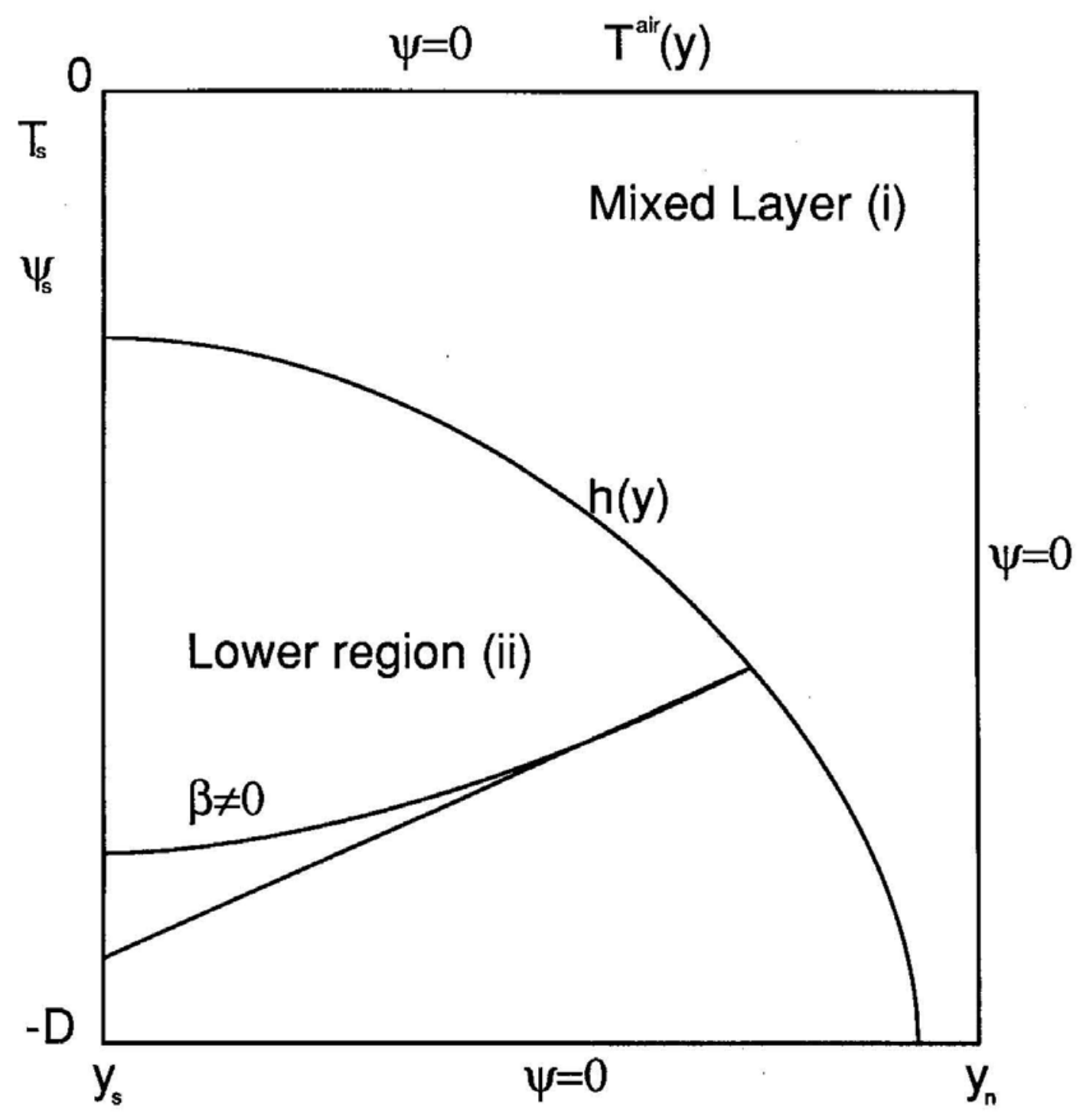

Figure (4.1). The schematic geometric configuration. The interface between the two layers is at depth $h(y)$ and reaches the bottom before the northern wall. Possible characteristics are presented in the lower domain. 
of time to reach it, so it has enough time to equilibrate its temperature with the air temperature.

(2) The surface heat flux is proportional to $T^{\text {air }}-T^{\text {surface }}$. The air temperature will be specified but the surface temperature is part of our solution; hence the flux is actually determined by the model.

(3) No flux through the bottom and northern walls.

(4) The integral constraint $\int_{-D}^{0} v d z=0=\int_{-h(y)}^{0} v d z+\int_{-D}^{-h(y)} v d z \quad$ is easily obeyed because we use the continuity equation (1.3) to define a stream function $\psi_{z}=-v$, and require $\psi=0$ along the top and bottom.

Two steps will be taken towards solving the problem. In the first, which we will refer to as the uncoupled problem, we postulate the momentum and heat budgets in each layer and solve for each region as a separate entity. For this part the stream function value along the interface $\left(\psi_{B}\right)$ is a free parameter and should be specified. It will turn out that in the upper layer we will have two coupled equations for the temperature and stream function and also one unknown boundary condition. Specifying $\psi_{B}$ is nothing but determining this condition, which in turn, enables us to solve the two equations. In order to solve for the lower layer one would need to know $\psi_{B}, T_{B}$, and $u_{B}$ (the last is the meridional velocity component). They can either be arbitrarily specified, or alternatively $\psi_{B}$ can be specified and the other two determined from the mixed layer solution.

In the last section of this chapter we deal with what we call the coupled problem. There, a scheme will be proposed where the upper and lower regions are coupled. The values of the temperature and velocity fields along the interface will no longer be arbitrarily specified but are computed as part of the solution. Mathematically, this problem is more difficult because the solutions in both regions are not independent. Moreover, additional information is needed (like $\psi_{s}^{(i i)}$ ) for the problem to be well posed. 
The heat and momentum budgets in each layer reflects our assumptions about the governing physical processes in each region. These mechanisms are of course independent of whether we choose to solve the coupled or uncoupled system. Thus, the same equations will be used in the two problems.

\section{Upper layer balances}

For the model to duplicate features of the circulations, it is convenient to treat the upper layer as a mixed layer where the temperature is approximately depth independent. The following sections illustrate the governing equations in that region.

\section{Heat equation}

The heat equation in the mixed layer is derived from the advection diffusion equation

$$
(v T)_{y}+(w T)_{z}=\kappa T_{z z}
$$

where the horizontal diffusivity was neglected with respect to the vertical one, and the continuity equation was used. The equation is integrated once in the vertical to give

$$
\int_{h(y)}^{0} d z(v T)_{y}+\left.w T\right|_{\text {bot }}=\left.\kappa T_{z}\right|_{\text {top }}
$$

where we let the interface be a function of latitude, assume that $w=0$ at the top, and that there is no diffusive flux through the interface. At this stage three steps are taken to simplify the equation.

(1) The velocity and temperature fields are split into mean $\left(^{-}\right)$and perturbation $\left({ }^{\prime}\right)$; $v=\bar{v}+v^{\prime}$, and $T=\bar{T}+T^{\prime}$. The primed fields are characterized by a zero vertical integral. The first term on the 1.h.s. is now written as

$$
-(\bar{v} \bar{T} h)_{y}+\frac{\partial}{\partial y} \int_{h(y)}^{0} d z\left(v^{\prime} T^{\prime}\right)+v_{B} T_{B} h_{y}
$$

where Leibnitz rule for differentiation was used, and, as always, the subscript $B$ denotes evaluation along the interface. 
(2) $w_{B}$ is evaluated from the vertically integrated continuity equation. The second term on the l.h.s. of (4.1) is now expressed as

$$
\frac{\partial}{\partial y}(\bar{v} h) T_{B}-v_{B} T_{B} h_{y} .
$$

(3) The r.h.s. is approximated by a Newtonian relaxation as $\chi^{\prime}\left(T^{\text {air }}-\bar{T}\right)$, with $\chi^{\prime}=$ $\chi^{\prime}(h)$ having the order of magnitude of $\kappa / h$.

Summing the three contributions, equation (4.1) takes the form

$$
-(\bar{v} \bar{T} h)_{y}+T_{B}(\bar{v} h)_{y}+h\left(\overline{v^{\prime} T^{\prime}}\right)_{y}=\chi^{\prime}\left(T^{\mathrm{air}}-\bar{T}\right)
$$

where $h\left(\overline{v^{\prime} T^{\prime}}\right)_{y}=\frac{\partial}{\partial y} \int_{h(y)}^{0} d z\left(v^{\prime} T^{\prime}\right)$. If we now assume that the temperature is approximately depth independent and replace $T_{B}$ with $\bar{T}$, the equation simplifies further to

$$
-h \bar{v} \bar{T}_{y}+h\left(\overline{v^{\prime} T^{\prime}}\right)_{y}=\chi^{\prime}\left(T^{\mathrm{air}}-\bar{T}\right)
$$

Vertically integrating the stream function defined by $-\psi_{z}=v$, and letting $\psi=0$ at the top leads to $\psi_{B}=-\bar{v} h$. If we also assume that the $\left(\overline{v^{\prime} T^{\prime}}\right)_{y}$ term is small compared to $\bar{v} \bar{T}_{y}$, we end up with

$$
\psi_{\mathrm{B}}(y) T_{y}=\chi^{\prime}\left(T^{\mathrm{air}}-\bar{T}\right) .
$$

Note, that this equation is general and holds for a non-constant layer depth. The main assumptions used to derive it were the smallness of the $\left(\overline{v^{\prime} T^{\prime}}\right)_{y}$ term, and the fact that the temperature is approximately depth independent. It can also be derived in an analogous fashion by writing the finite difference heat and mass conservation equations for a fluid column.

For scaling purposes we make use of two known values: $T_{n}^{\text {air }}$ which is the air temperature at the northern wall, and $T_{s}$, the temperature of the incoming flow through $y_{s}$. The scaled equation is

$$
\psi_{\mathrm{B}}(y) T_{y}=\chi\left(\frac{T^{\mathrm{air}}-T_{n}^{\mathrm{air}}}{T_{s}-T_{n}^{\mathrm{air}}}-\bar{T}\right) .
$$


Here, $T$ is $O(1), \chi=\frac{\chi^{\prime} L}{V H}$, and $V$ is a scale for the northward velocity. The coefficient $\chi$ can be interpreted as

$$
\chi=\frac{\kappa L}{H^{2} V}=\frac{L / V}{H^{2} / \kappa}=\frac{\text { Time scale for horizontal motions }}{\text { Time scale for vertical diffusion }} .
$$

This usually gives a small number in the interior, and a larger one in the mixed layer.

\section{Momentum Balance}

Dissipation of momentum in the mixed layer is postulated in the simplest way by introducing linear drag in the momentum equations, viz:

$$
\begin{aligned}
-f v & =-r u \\
\rho_{0} f u & =-p_{y}-\rho_{0} r v \\
0 & =-p_{z}+\rho_{0} g \alpha T-\rho_{0} r w \\
v_{y}+w_{z} & =0
\end{aligned}
$$

leading to

$$
r^{2} \psi_{y y}+\left(r^{2}+f^{2}\right) \psi_{z z}=r \alpha g T_{y}
$$

Our system is now composed of two equations (4.3) and (4.5) relating the temperature and stream function in the upper layer. The boundary condition $\psi_{B}$ is also unknown, but the incoming flow field, its temperature, and its depth are specified. The $u$ velocity is determined from (4.4a) once the stream function is found.

At instances where we do not want to have dissipation where there is no shear in the flow, it is appropriate to replace the linear drag with a $\nabla^{2}$ operator. The linear drag was chosen here mainly to simplify the equations. In addition, because of the geometry of the system, there is always shear in the upper layer stream function. If we decide to make the substitution, (4.5) will be of higher order $\left(\nabla^{4} \psi\right.$ instead of $\left.\nabla^{2} \psi\right)$, the equation will remain elliptic and the solutions would probably not change much. 


\section{Lower layer balances}

The lower region represents the interior fluid (region ii) that lies under the mixed layer. In general, the viscous forces there are small and often are completely ignored.

Heat budget

Assuming we have no dissipation of heat, the equation takes the simple form of $v T_{y}+$ $w T_{z}=0$, which can be concisely written as

$$
J(\psi, T)=0 .
$$

\section{Momentum balance}

The momentum budgets of the upper layer are general enough and can be successfully applied in the lower layer. Because we want to look at the limit where $r \rightarrow 0$ we keep the same $y$ and $z$ equations (4.4b) and (4.4c) but add the nonlinear terms to the $x$ momentum balance. This way the meridional velocity would not vanish when friction does. (In the upper layer, dissipation is important and balances the $f v$ term, in the interior, frictional forces are much weaker and inertial terms are more important.) If we define the quantity $m=u-f y$, we can write the new $x$ momentum balance that replaces $(4.4 a)$ as

$$
J(\psi, m)=-r u
$$

where the rest of (4.4) is still applicable. The quantity $m$ is important because it represents the angular momentum of the system. The equivalent of (4.5) will now be

$$
r \nabla^{2} \psi=f m_{z}+\alpha g T_{y}
$$

Note, that unlike the mixed layer, the lower region is described by three statements (and not two) (4.6), (4.7) and (4.8) relating $m, \psi$ and $T$. 
The pure thermal wind case is a result of taking the limit of $r \rightarrow 0$, thus reducing the set to geostrophy in $y$ and hydrostatic balance in $z$. The deviation from hydrostatic balance is usually negligible because it enters the scaled equation via the $\psi_{y y}$ term. This contribution is smaller than the $\psi_{z z}$ one by order aspect ratio squared. The limit of $r=0$ turns out to be of particular interest in light of the fact that the interior fluid will conserve both temperature and angular momentum. The results will be rather surprising.

\section{A more general model for the mixed layer}

Before we start analyzing the proposed model for the northern part of the circulation we would like to present a more complete picture of the full scale gyre. Consider the configuration of figure (4.1) in a global circulation problem with a large distance between the two side walls. As before, the ocean is divided in the vertical into two domains, with a mixed layer on top of the interior fluid. The shape of the mixed layer is a function of latitude-either known or determined as part of the solution. For this geometry, the formal presentation of the dynamics in the mixed layer is now a little bit different than before, but that in the lower layer is unchanged.

The heat balance will be the same as in (4.1) with an additional term representing the fact that in the region of incoming flow into the mixed layer there is a contribution to the global heat balance from advection of heat into the layer. This effect is enhanced as the temperature discontinuity at the base of the mixed layer becomes larger. The heat flux into the mixed layer through the lower boundary is $w T_{z}$, with $w$ denoting the vertical velocity at the base of the mixed layer, and $T_{z}$ the local vertical temperature gradient. We can approximate these terms as $w T_{z}=\frac{w}{h(y)}\left(\bar{T}-T^{\text {int }}\right)$ with $\bar{T}$ as the mixed layer temperature (which is approximately depth independent) and $T^{\text {int }}$ the 
interior temperature below the mixed layer. If we parameterize the Reynolds fluxes as was done for (4.1), we get the following relation to replace (4.2)

$$
\psi_{B}(y) \bar{T}_{y}=\frac{\kappa}{h(y)}\left(T^{\mathrm{air}}-\bar{T}\right)+\psi_{B y}\left(\bar{T}-T^{\mathrm{int}}\right) \mathcal{H}(\operatorname{sign}(-w))
$$

with $\mathcal{H}(\operatorname{sign}(-w))$ as the Heaviside step function emphasizing the fact that the advective contribution is available only where there is incoming flow at the base of the mixed layer. The complexity of the problem increases not only because the depth is now latitude dependent, but also because the coupling between the solution in the two layers is much stronger due to the $T^{\text {int }}$ term.

The momentum balances are similar to (4.4), but now we have to include stress in the equations. If we choose to ignore all stress variations except in the vertical, the only contribution will be to the $y$ momentum equation (4.4b), that will now be

$$
f u=-p_{y}-r v+\frac{\partial \tau^{w}(y)}{\partial z}
$$

where $\tau^{w}(y)$ is the $y$ component of the stress. The superscript $w$ indicates that for most cases, the stress is simply the wind stress. As was done with the vertical fluxes, we assume that the stress vanishes at the bottom of the mixed layer. The stress, or more generally, even a fluctuating forcing that has zero mean stress, is essential in the formation of the mixed layer in southern regions because without it the balance between the upward flow $\left(w T_{z}\right)$ and the downward diffusion $\left(\kappa T_{z z}\right)$ leads to an exponential temperature profile. It is the addition of downward mixing of momentum that contributes to the depth independent temperature profile in the mixed layer. From (4.4) $c-d$ and (4.10) we generate the vorticity equation, which looks like (4.5) with one added term

$$
r^{2} \psi_{y y}+\left(r^{2}+f^{2}\right) \psi_{z z}=r \alpha g T_{y}-r \frac{\tau^{w}(y)}{h(y)}
$$

where as before we approximated the $z$ derivatives by $1 / h(y)$. Ideally, one would like to find a third equation for the depth, thus, having a set of three equations for $\psi, T$, 
and $h(y)$. After doing so, the system is coupled to the heat and momentum budgets of the lower layer, and is sufficient in determining the temperature, velocity and mixed layer depth everywhere in both layers. Technically, instead of solving a set like that, it is often simpler to proceed in an iterative fashion by assuming an interface profile and solving for the temperature and stream function fields in both layers. The matching across the interface will often not be possible unless the right shape was assumed. It has been our experience with similar problems that this procedure does not often converge, and when it does, the convergence is very slow. Be that as it may, it is not very clear how to write an equation for the mixed layer depth. Even for the simple case where only diffusion was present [equation (2.4)] we did not have an analytic expression for the interface, but its shape was computed numerically by many iterations. In this formalism the problem is harder. A possible approach is to write the equations with the use of convective overturning, thus, keeping the $T_{z}$ term, but making it vanishingly small if the stratification is unstable. This way we might be able to compute the shape of the interface numerically is a fashion similar to that used in the previous chapter.

Due to the complexity of the problem and our main interest in the northern region, we decided to limit our attention to the sinking region and replace the southern extent of the domain by some proper matching boundary conditions imposing incoming flow into the mixed layer and outgoing flow from the lower region. 


\section{The uncoupled problem}

\section{Analysis of heat budget in the mixed layer}

The temperature structure in the upper layer can be found from (4.3) in two ways. The simplest is to specify it along the interface; then, because the temperature is depth independent, this actually determines it everywhere. $\psi_{B}$ is found immediately to be

$$
\psi_{B}(y)=\frac{\chi}{T_{y}}\left(\frac{T^{\mathrm{air}}-T_{n}^{\mathrm{air}}}{T_{s}-T_{n}^{\text {air }}}-\bar{T}\right)
$$

Thus, we establish all the necessary boundary conditions needed to solve (4.5) for $\psi$. The last is an elliptic equation, so specifying the stream function around our domain, and knowing the forcing $\left(\propto T_{y}\right)$ is sufficient to determine the velocity structure everywhere in the upper layer (albeit not too easy if the geometry is not rectangular). Although by far the simplest way, one would not be automatically able to satisfy the condition $\psi_{s}\left(y=y_{s}\right)=\psi_{s}^{(\mathrm{i})}\left(z=-h\left(y_{s}\right)\right)$. For example, take a linear relation for both $\bar{T}(y)$ and $T^{\text {air }}$. Let $y=0$ be the latitude of the incoming flow, and $y=1$ that of the northern boundary. Assume a simple linear relation $\bar{T}=1-y$, so $\bar{T}(0)=1$ and $\bar{T}(1)=0$, and also that $T^{\text {air }}=b(1-y)$. Specify $\psi_{s}^{(\mathrm{i})}\left(z=-h\left(y_{s}\right)\right)=1=\psi_{\mathrm{B}}(0)$ and $\psi_{B}=0$ along the northern wall. The equation above yields $\psi_{B}=\chi(1-y)\left(1-\frac{b}{T_{B}}\right)$. ( $T_{\text {s }}$ will usually be larger than $b$.) The condition $\psi_{B}(1)=0$ is satisfied, but in order to have $\psi_{B}(0)=1$, we should have $\chi\left(1-\frac{b}{T_{s}}\right)=1$, relating the parameters $\chi, T_{s}$ and $b$. Physically, these parameters correspond to the diffusivity, incoming temperature, and air temperature, and thus are independent.

This example can be viewed as a statement that not all initial guesses of $\bar{T}$ (or $\psi_{B}$ ), are physically meaningful for the upper layer. Only those that will yield the 'proper' values for $\psi_{B}$ (or $\bar{T}$ ) near the northern and southern boundaries are plausible. The problem obviously gets more complicated when we take into account the coupling between the lower and upper layers. 
The second and more physically intuitive way involves solving for $\bar{T}$ when $\psi_{B}$ is specified. For that problem we can look at (4.3) as a first order linear equation in the temperature, but with two boundary conditions that should be met; $\bar{T}=T_{s}$ for the incoming flow, and $\bar{T}=T_{n}^{\text {air }}$ at the northern boundary. How can this be done for a first order problem? By the special nature of the equation we are assured that since $\psi_{\mathbf{B}} \rightarrow 0$ approaching $y=y_{n}$, the temperature will converge to the air temperature. Thus, only one boundary condition is left free, namely, $\bar{T}=T_{a}$ at the southern boundary. The solution to the non-homogeneous linear equation can be written as

$$
\bar{T}(y)=\frac{1}{I(y)}\left[T_{s}+\int^{y} \frac{\chi}{\psi_{B}(t)} \frac{T^{\mathrm{air}}(t)-T_{n}^{\mathrm{air}}}{T_{s}-T_{n}^{\text {air }}} d t\right]
$$

with $I(y)=e^{x \int^{y} \frac{d t}{\phi_{B}(t)}}$. For simple linear relation $T^{\text {air }}=b(1-y)$ and $\psi_{B}=1-y$ (leading to a constant $w$, but not necessarily constant flux through the interface) the solution for the temperature is

$$
\bar{T}(y)=(1-y)\left[T_{s}+\frac{\chi b}{T_{s}} y\right]
$$

which is a quadratic polynomial in $y$. 


\section{Momentum budget and solutions for the upper layer}

The momentum balance (4.5) enables us to determine the stream function everywhere once the temperature is known. But in order to calculate the last, the stream function along the interface had to be given, so now we know the boundary condition all around the upper layer and the solution to the elliptic problem can be determined. Equation (4.5) can be scaled and simplified by noting that based on the boundary conditions there are two possible scales for $v$. The first is derived from the magnitude of the specified incoming flow, $V_{s}=\psi_{s}^{(i)} / H$, while the second results from the thermal forcing $V_{T}=\alpha g H\left(T_{s}-T_{n}^{\text {air }}\right) / r L$. [The proper scale for $u$ from (4.4a) is $U=V f / r$ and depends on our choice of the scale for the northward velocity component.]

Because $L / H$ is greater than unity, we can neglect the $\psi_{y y}$ term in (4.5), and with the use of the last two scales its dimensionless form becomes

$$
\left(1+\frac{f^{2}}{r^{2}}\right) \psi_{z z}=\frac{V_{x}}{V_{s}} T_{y}
$$

which simplifies to $\psi_{z z}=\gamma T_{y}$ with

$$
\gamma=\left(T_{s}-T_{n}^{\mathrm{air}}\right) \frac{\alpha g}{V} \frac{H}{L} \frac{r}{\left(r^{2}+f^{2}\right)} .
$$

Looking at $\gamma$ as a function of $r$ we find that the maximum of the quadratic function is reached when $r=f$. This means that if we fix the forcing $\left(V_{T}\right.$ and $\left.V_{s}\right)$, and choose to look at the friction as a variable, there is a region where two frictional parameters gives the same ratio $\gamma$. One value of $r$ is larger than the Coriolis parameter, and one smaller.

The ratio $\gamma$ gives rise to two possible balances. The strong thermal forcing balance yields $\psi_{z z}=T_{y}$, giving $\psi=\left(T_{y}\right) z^{2} / 2+a(y) z+b(y)$. Matching the boundary conditions at the top $\psi(z=0)=0$ yields $b(y)=0$, while the bottom one $\psi(z=-h(y))=\psi_{B}(y)$ gives $a(y)=\frac{\psi_{B}(y)}{h(y)}-\frac{h(y)}{2}\left(T_{y}\right)$; thus, we end up with

$$
\psi(y, z)=\frac{1}{2}\left(T_{y}\right) z(z-h)+\frac{z}{h} \psi_{B}(y)
$$


and we still have two more boundary condition to meet. Along the northern wall we should have $\psi\left(y_{n}, z\right)=\psi(1, z)=0$, and at the southern boundary we want $\psi\left(y_{s}, z\right)=$ $\psi(0, z)=\psi_{s}(z)$. These conditions require boundary layers along the two walls, as is expected when we neglect the highest order derivative with respect to $y$ in equation (4.5).

The weak thermal forcing case is written as $\psi_{z z}=0$, which after matching the boundary conditions at the top and bottom yields

$$
\psi(y, z)=\frac{z}{h} \psi_{B}(y)
$$

This solution is actually the last term of the previous expression and differs from it among other reasons because it satisfies the condition on the northern wall, thereby requiring a boundary layer at $y=0$ only. [The previous solution had $\psi_{B}(1, z)=0$, but $T_{y}(y=1)$ does not necessarily vanish.]

If we take as typical values, $r=10^{-5}, \quad f=10^{-4}, \quad \alpha=10^{-4}, \quad g=10^{3}$, and $\left(T_{s}-T_{n}^{\text {air }}\right)=1$, we get that for $\gamma=1$ we need $V \approx 2 \mathrm{~cm} / \mathrm{sec}$, and $L / H \approx 50$. So for this range, the first balance explored is the key, because both $\psi_{z z}$ and the $T_{y}$ terms are larger than unity. Alternatively, if $V$ is larger, $L / H$ is bigger, or the dissipation is weaker, $\gamma$ decreases, and the second balance is applicable. A first order balance that includes the $\psi_{y y}$ term is only possible for order one aspect ratio, and appropriate values for $V$ and $r$-depending if we want to include the $\psi_{z z}$ term, the $T_{y}$ term, or both. In the last possible balance where $\gamma \gg 1$ we have to have a different scale for $\psi$ because otherwise we get $T_{y}=0$ leading to $T=T(z)$ which is not likely for a mixed layer. Either $(4.13 a)$ or $(4.13 b)$ can now be coupled to the heat equation (4.3) to form a complete set for $\psi$ and $T$.

Despite the previous computations, the momentum equation is not very exciting and only enables us to find the velocity field in the mixed layer after $\psi_{B}$ is specified. With the possible exception of the boundary layers next to the northern and southern 
walls, typical fluid trajectories resemble the first quarter of an arc. The contribution of this equation to the global flow field will be more important in the coupled problem because there we will not have the freedom to choose $\psi_{B}$ as we please.

\section{Lower layer conservation constraints}

The formulation used in the previous section contrasts the balances in the two layers, in particular the differences in the heat budgets. The upper layer acts as a mixed layer where the temperature is homogeneous in the vertical, while dissipation of momentum was postulated via linear friction. In the lower layer, frictional and dissipative processes are minute. We start by ignoring them altogether, and find out that the velocity field has a very surprising shape. The importance of the weak dissipative processes in altering this shape is investigated. 
The potential vorticity

For a nonlinear system that is symmetric in $x$ and has no frictional forces we can write the $x$ momentum, heat, and continuity equations as

$$
\begin{array}{r}
m_{t}+v m_{y}+w m_{z}=0 \\
T_{t}+v T_{y}+w T_{z}=0 \\
v_{y}+w_{z}=0
\end{array}
$$

where $m=u-f y$ is the angular momentum of a fluid particle. We define $q=$ $m_{z} T_{y}-m_{y} T_{z}$ and after a short manipulation get

$$
\left(\frac{\partial}{\partial t}+\vec{v} \cdot \nabla\right) q=0
$$

This is exactly the conservation of potential vorticity $\left(q=u_{z} T_{y}-u_{y} T_{z}+f T_{z}\right)$ with temperature as a conservative tracer. The major difference between this derivation and the conventional one is the lack of $y$ momentum equation. Thus, we conclude that the set $J(\psi, T)=0$ and $J(\psi, m)=0$ is equivalent to conserving the potential vorticity. One should also note that the small Rossby number approximation $(\epsilon \ll 1)$ of $J(\psi, q)=0$ does not lead to $J\left(\psi, T_{z}\right)=0$, because in our case $\partial_{x}=0$, so the $x$ momentum balance $\epsilon\left(v u_{y}+w u_{z}\right)-f v=0$ yields $v=0$ to lowest order, and we have to assume that at least one of the nonlinear terms is big enough so that our zonal flow Rossby number is of order one. Therefore, we cannot neglect all the advective terms relative to the Coriolis one. 


\section{Non-dissipative fluid}

The addition of the thermal wind equation to the conservation statements of heat and angular momentum closes the set (4.14) completely

$$
m_{z}=-\frac{\alpha g}{f} T_{y} .
$$

Fortunately, this system of equations can be easily analyzed despite its nonlinearity. Our conservation statements implies that $m$ and $T$ are functions of $\psi$ only. Let us write these functions as $T=T(\psi)$ and $m=\mathcal{M}(\psi)$. Substituting in the thermal wind relation gives

$$
\psi_{y}+\frac{f \mathcal{M}^{\prime}}{\alpha g T^{\prime}} \psi_{z}=0
$$

where primes denote differentiation with respect to $\psi$. This hyperbolic equation is solved by characteristics. It is more transparent to write the last equation as the set

$$
\begin{aligned}
& \frac{d z}{d y}=\frac{f}{\alpha g} \frac{\mathcal{M}^{\prime}}{T^{\prime}} \\
& \frac{d \psi}{d y}=0 .
\end{aligned}
$$

From the first of these equations we know that the slope of the characteristics depends on the stream function alone because both $\mathcal{M}^{\prime}$ and $\tau^{\prime}$ are functions of $\psi$ only. The second is simply stating that the stream function is constant along the characteristics. Thus, even without the full solution we know that the characteristics-which are also the fluid parcel trajectories-are straight lines. Physically what we have is a situation where a fluid parcel must follow a straight line path if it is to conserve its temperature and angular momentum (or equivalently, its vorticity). Along this path $\psi, T$, and $m$ are fixed, and the slope of the line is determined once these three quantities are given along the interface. The fact that the fluid trajectories are straight was derived here with no reference to the shape of the interface, and is a direct result of the dynamics applied. It is quite unexpected because the conservation of potential vorticity is favored 
for general circulation problems, where the fluid path is far removed from a straight line.

Some simple consequences can be drawn before attempting to solve for any particular problem. Let us examine the characteristics in figure (4.1). If we do not want the northernmost one to intersect the bottom (in cases where the bottom layer has a finite depth), we must have both a sufficiently deep lower layer, and a moderate slope. To the north and below of this characteristic the fluid is practically stagnant, and does not participate in the circulation scheme. As we move southward, the slope of the characteristics must decrease monotonically, otherwise they will intersect, which would imply that there are regions in the fluid where two stream function values are possible.

The region where the fluid trajectories are pointing straight down must be limited in its extent because we do need to close the vertical circulation cell and bring the fluid back into the mixed layer where it will advance northward into the sinking region. At first glance one could think that a possible exception where no bending of fluid trajectories is needed is the situation where the mixed layer deepens to the north with a monotonically increasing slope as in figure (4.1). Thus, it is possible to have straight characteristics all of which have trajectories pointing upward. In this description we can theoretically get all the fluid back into the mixed layer, but still have a large portion of the fluid-that that lies between the characteristic emanating from the northernmost deepest point and the lower boundary-where again the fluid is motionless. However, it is not clear that those upward trajectories can even exist. The slope of the characteristics depends on the ratio between $\mathcal{M}^{\prime}$ and $T^{\prime}$ on the interface. In order to have upward trajectories for some cases and downward for others, we must change the sign of this ratio. As one progresses northward along the interface the stream function and temperature decreases, and probably the angular momentum has the same tendency. But even if does not, it is hard to envision it being positive for certain interfaces and $\psi_{B}$ values, and negative for others. In addition, our experience 
from numerical simulations indicate that the characteristics point downward for a wide range of imposed initial conditions.

As with other problems that are solved using characteristics, if one knows the values of the variables $\psi, m$, and $T$ along a particular line from which the characteristics emanate, it is possible to find them at other locations. But one should be aware of the difference between the direction of the characteristics, the passage of information flow in the system, and the sense of the flow field. For example, consider free geostrophic flow, the characteristics of which are lines of constant potential vorticity, or latitude lines if the depth is constant. These trajectories originate at the eastern boundary and travel westward. Sverdrup flow (which is really not a case of free flow because the curl of the wind stress is a source of vorticity) does not follow these characteristics. Moreover, in the southern part of the subpolar gyre and the northern region of the subtropical gyre, the sense of the circulation opposes the direction of the characteristics.

We will show later, that although in our lower layer the streamlines follow the characteristics, the information flow in the system is carried in the opposite way. This means that even although the characteristics go from the interface to the southern wall, and the sense of the circulation is clockwise, the information travels from the southern wall towards the interface. Graphically, one can interpret the statement as saying that the outgoing flow affects the circulation next to the interface. This phenomena is exemplified when boundary layers are generated, and indeed we will see later on that their location is always next to the interface rather than next to the southern boundary. 
The beta effect

As was done before when the generalized mixed layer model was introduced, we extend the problem to a global circulation scale (although we only only look at characteristics emanating from the sinking region) and examine the behavior of the potential vorticity conserving fluid. Equations (4.16)-(4.18) are still valid, but now we use the $\beta$ plane approximation so $f=f(y)$. The slope $d z / d y$ is not constant along the characteristics any longer, but varies with $y$. Because the Coriolis parameter is a monotonically decreasing function of $y$ as we go southward, the new slope of the characteristics will have the same feature, as shown in figure (4.1).

The exact solution is derived in the standard fashion. Use $f=f_{0}+\beta y$ and integrate (4.18a) to get the shape of a typical characteristics that intersect the interface at location $\left(y_{0}, z_{0}\right)$

$$
z=z_{0}+\frac{\mathcal{F}\left(\psi_{0}\right)}{\alpha g}\left[f_{0}\left(y-y_{0}\right)+\frac{1}{2} \beta\left(y^{2}-y_{0}^{2}\right)\right]
$$

where we used the fact that because $\psi$ is fixed along the characteristics, $\mathcal{M}^{\prime} / T^{\prime}=\mathcal{F}(\psi)$ can be evaluated anywhere along the trajectory, in particular at $\left(y_{0}, z_{0}\right)$ where $\mathcal{F}\left(\psi_{0}\right)$ is known. A typical characteristic is presented in figure (4.1). Note, that we were indeed able to bend the trajectory, but not enough. Its slope will never be less than zero, so that a fluid parcel along it will never move upward unless it had an upward motion to begin with, (a result we already rejected) in which case, the slope of its trajectory will decrease.

The derivation above is yet another support of the observation that even on a large scale, the absence of friction and diffusion leads to a flow that only goes down towards the bottom and never rejoins the mixed layer. We must therefore conclude that there are regions where the dynamics are different from those of equations (4.14)-(4.16). 


\section{Additional results for angular momentum conserving flow}

The conservation of potential vorticity in a system symmetric in its third dimension has additional implications. Envision a rotating stratified fluid with a thin mixed layer on its top. Assume the value of the meridional velocity is known somewhere. The conservation of angular momentum $m=u-\frac{1}{2} \beta y^{2}$ states that at a location $5000 \mathrm{~km}$ away, this velocity should change by more than a hundred meters per second. This in itself really prohibit the circulation from extending over such distances. But even if it does not, and the gyre is not confined to a narrow latitude bend, a fluid parcel that re-enters the mixed layer with this high velocity has to dissipate its excess momentum along its route north before it rejoins the interior circulation. This would usually lead to very high mixing coefficients in the upper layer. Again this justifies our conclusion that there are regions in our flow where the angular momentum is not conserved. 


\section{Effects of dissipation on the fluid trajectories}

Let us look closely at the addition of friction to the straight characteristics case. Remember though, that a fluid particle still conserves its potential vorticity because both angular momentum and temperature (4.14) can still be a function of $\psi$ only, while the dissipation is included in the $y$ momentum equation (4.4b). The boundary conditions are still $\psi=0$ along the northern and bottom walls. Using the conservation of heat and angular momentum (4.8) is rewritten as

$$
r \nabla^{2} \psi=f \mathcal{M}_{\psi} \psi_{z}+\alpha g \tau_{\psi} \psi_{y}
$$

In the following analysis it is assumed that $\mathcal{M}$ and $\tau$ are linear function of $\psi$, and thus the above equation is written schematically as

$$
r \nabla^{2} \psi=\psi_{z}+a \psi_{y}
$$

where $a$ is a constant. For the purpose of examining the importance of the elliptic term to the otherwise hyperbolic equation, the conditions on the remaining two boundaries should be specified. This is done by assuming that the stream function along the top of the lower domain (which is the interface) and southern walls is linear in $y$ and $z$, respectively ( $\psi$ at the top left corner is 1 ). For simplicity, we also let the interface be flat (the only case in the chapter) so that the lower layer has a rectangular shape. The results are drawn in figures (4.2)a-k, where the relevant parameters are summarized in 
table (4.1).

\begin{tabular}{|c|r|r|}
\hline Figure & \multicolumn{1}{|c|}{$\mathrm{a}$} & \multicolumn{1}{|c|}{$\mathbf{r}$} \\
\hline $\mathrm{a}$ & 10,000 & 100 \\
$\mathrm{~b}$ & 100 & 100 \\
$\mathrm{c}$ & 100 & 1 \\
$\mathrm{~d}$ & 2 & 1 \\
$\mathbf{e}$ & 1 & 1 \\
$\mathrm{f}$ & 0.1 & 1 \\
$\mathrm{~g}$ & 2 & 0.01 \\
$\mathrm{~h}$ & 1 & 0.01 \\
$\mathrm{i}$ & 0.1 & 0.01 \\
$\mathrm{j}$ & 0.01 & 0.01 \\
$\mathrm{k}$ & 0.1 & 0.005 \\
\hline
\end{tabular}

Table(4.1)

As mentioned before, it is interesting to note that for the cases that exhibit a boundary layer character, the layer is never at the southern boundary, but rather along the interface. This indicates that the information along the characteristics passes from the outflow boundary to the interface, opposite to the regular notion that the information advances with the flow field. It is easier to understand the phenomena by treating (4.20) as an advection diffusion equation. Note, however, that $\psi$ is now the tracer field while the velocity is given by $v=\alpha g \tau_{\psi}$ and $w=f M_{\psi}$. Along the interface (and everywhere else in the fluid) $\psi, T$, and $m$ decrease as we go northward, thus, $\tau_{\psi}>0$ and $\mathcal{M}_{\psi}>0$, and hence $v$ and $w$ are positive. This shows that for the velocity associated with our tracer field, upstream means northward and up, and the information goes from the southern wall to the interface. The location of the boundary layer is governed by the difference between the intersections of the fluid trajectories leaving the southern wall, and the boundary conditions imposed on the interface.

Looking at the pictures, the following observations can be made:

1) Case (' $h$ ') is basically the straight characteristics case with a slope of unity.

2) The cases with the very large slopes ('a','c','g') are those with large $a$, and can be approximated to first order by $\psi_{y}=0$. A strong boundary layer in $z$ near the top 

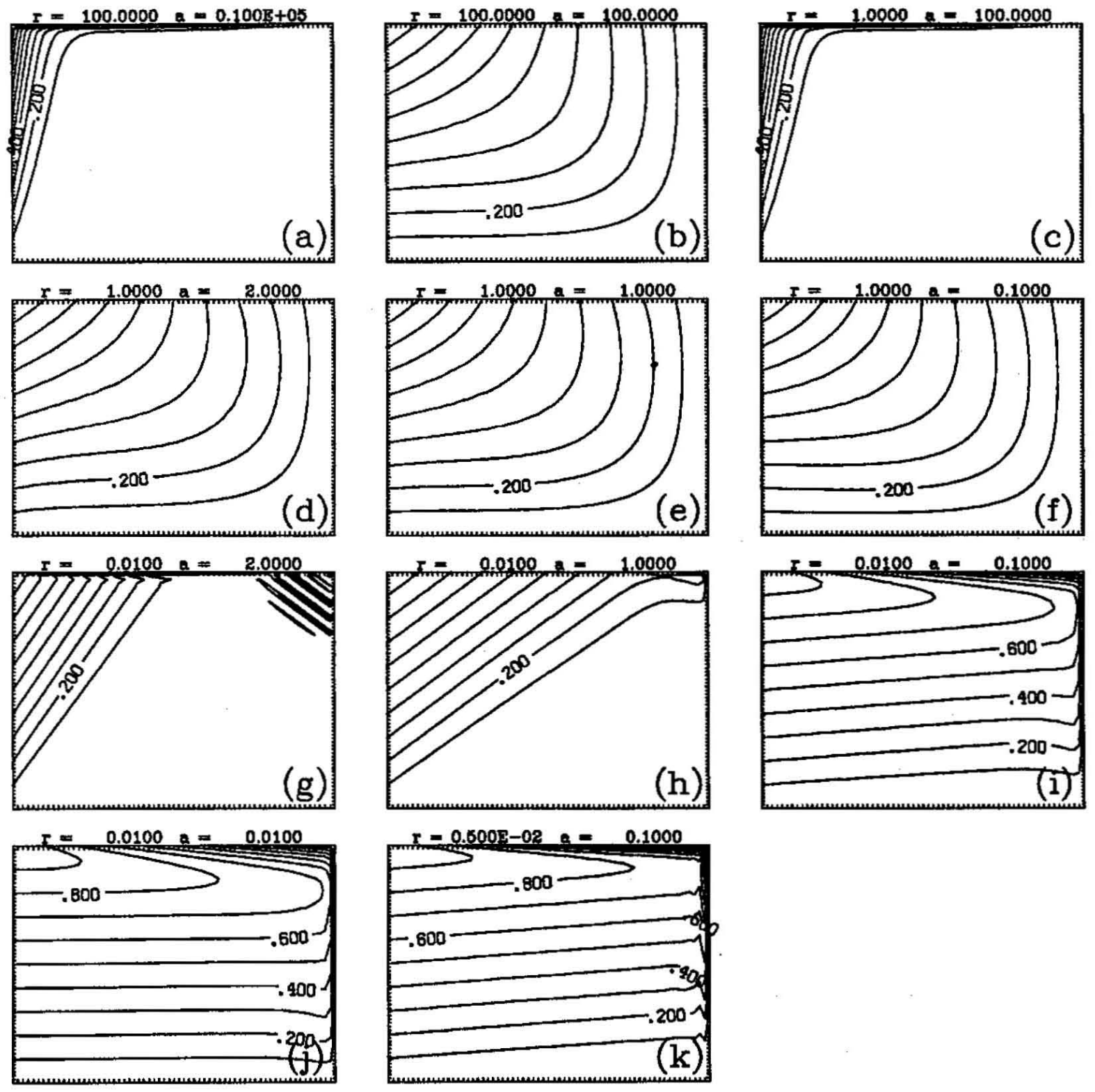

Figure (4.2). Numerical solutions for the stream function in the lower layer as a function of $a$ and $r$ in equation (4.20). (a) $r=100, a=10,000$. (b) $r=100, a=100$. (c) $r=1$, $a=100$. (d) $r=1, a=2$. (e) $r=1, a=1$. (f) $r=1, a=0.1$. (g) $r=0.01, a=2$. (h) $r=0.01, a=1$. (i) $r=0.01, a=0.1$. (j) $r=0.01, a=0.01$. (k) $r=0.005, a=0.1$. 
boundary is apparent in those cases. The flow in the interior is weak. Note, that in cases ' $\mathrm{b}$ ' and ' $\mathrm{d}$ ', although $a$ is big as in 'c' and ' $\mathrm{g}$ ', respectively, the friction enters the zero order balance.

3) Strong friction as in cases ('b', $d$ ', 'e', $f$ ') bends the streamlines. This results from the fact that the solution to $\psi_{y y}+\psi_{z z}=0$ is proportional to $y z$. The streamlines close to the upper left corner are almost straight.

4) In the last three cases (' $\mathrm{i}$,' $\mathrm{j}$ ', ' $\mathrm{k}$ ') the $\psi_{z}$ term dominates the balance, thus, there is a $y$ boundary layer near the northern wall, and the streamlines cross the interior almost horizontally, since $a \ll 1$.

The figures indicate that the two parameters $a$ (which relates to the values of $\psi$, $T$, and $m$ along the interface) and $r$ (the interior friction) are important in determining the shape of the streamlines. When $a / r<1$ the dissipation is important and the streamlines are curved. At instances where the elliptic term is small, $(a / r \gg 1)$ the trajectories are straight ('a','c','g',' $h$ ', 'i','j', 'k'). The slope of the characteristics is $a$, so that when $a \gg 1$ ('a', 'c', 'g') there must be a boundary layer next to the interface. In this layer fluid moves southward until it reaches the proper point where it can leave the boundary and go to the southern wall in a trajectory with a sharp inclination. If $a$ is large enough fluid parcels will leave this boundary layer only near the southern wall; thereby, the vast majority of the fluid volume remains motionless. For weak slopes (' $i$ ', $\mathrm{j}^{\prime}, \mathrm{C}^{\prime} \mathrm{k}$ ) there is a top boundary layer in which the fluid moves northward, and a northern boundary layer where it goes down. The trajectories are almost horizontal between that wall and the southern extent of the domain.

It is important to remember that the inclusion of friction changes the problem completely, and requires specifying boundary conditions on all surrounding walls, whereas the hyperbolic problem alone requires only one boundary condition, on the boundary from which the characteristics emanate. The requirement on the additional three boundaries are as follows. Along the northern wall the value for the stream function 
is fixed, and is taken to be the same as at the point of intersection between it and the interface. This choice bears no effect on the solution, since no characteristics emanate from this wall. The conditions on the southern wall are kept free, and when there is no friction, are determined by the characteristics intersecting it. A potential problem lies with the bottom boundary. On one hand we want to keep the stream function value there the same as along the northern wall-say zero-but on the other hand we might have characteristics which are steep enough to intersect it. One way to overcome the problem is simply to move the bottom downward; alternatively, for a given bottom depth there is a maximum slope value (4.18a) for which a characteristic goes from the top right corner to the bottom left one. For all cases where this slope is reached somewhere along the interface, but not at the northernmost point, there will be an intersection with the bottom, requiring changes in the dynamics to construct a boundary layer there. 


\section{Discussion of the coupled problem}

The coupled problem is just a combination of the upper and lower region solutions to form a part of the vertical circulation cell. The fluid enters the mixed layer and goes down into the interior where it flows southward. Somewhere in a region not treated in the model the water re-enters the mixed layer again and moves northward to complete the cell.

Assuming there is no friction in the interior it is easy to combine the previous solutions. The temperature $T_{\mathrm{s}}^{(\mathrm{i})}$ and velocity $\psi_{\mathrm{s}}^{(\mathrm{i})}$ of the incoming flow are specified, as well as the air temperature. For any guess of $\psi_{B}$, (say linear in $y$ to get constant downwelling) we solve for the upper layer completely. (4.3) is used to determine $\bar{T}$ and (4.4a) to get $m$. Once these are determined, we know the slope of the characteristics, and so $\psi, m$, and $T$ are known everywhere in both layers. Solutions where the characteristics are very steep and intersect the bottom can be rejected and a different guess for $\psi_{B}$ can be made, leading to more moderate slopes.

When there is friction in the lower layer, the complexity of the system increases. It was shown that the information in the system is moving opposite to the circulation, and that a boundary layer may be formed next to the interface. We now can say that the conditions for the outgoing flow $\psi_{b}^{(\mathrm{ii})}$ are controlling those of the incoming one, and vice-versa. The solution to the problem can be carried out in an iterative way. As before, $\psi_{\mathrm{B}}$ is assumed and the upper layer is solved. Conditions at the outgoing boundary are also assumed and (4.20) is solved for the interior region. A solution where the characteristics do not match smoothly to the interface is rejected and another guess for $\psi_{s}^{(\text {ii) }}$ is made. We continue to improve the guess until a solution without a boundary layer is reached. Alternatively, we can fix $\psi_{s}^{(\text {ii) }}$ and change $\psi_{\mathbf{B}}$ until we find a solution where no boundary layer exists in the interior.

In the two problems described above there still is a lot of freedom even though the solutions in the upper layer determine the shape of the characteristics in the lower 
one. We are still free to choose $\psi_{B}$ as we please, and presumably there will be a family of values that will lead to reasonable solutions. A system where the values along the interface are part of the solution is probably more realistic but harder to realize analytically and numerically.

\section{Interior constraints}

In order to limit our freedom in choosing $\psi_{B}$ but still drive the coupled system by conditions at the incoming end we use simple conservation statements at the outgoing end, thereby arriving at conditions relating $\psi$ and $T$ there. Imposing those additional constraints is in many ways equivalent to an additional boundary condition, so now not all guesses of $\psi_{B}$ are valid, but only those that lead to solutions with specific relations between $\psi$ and $T$ at certain locations. The reasons we chose the southern boundary as the position to impose these additional conditions are two-fold. Not only do the conditions at that latitude have to match to the rest of the vertical gyre, but because of the direction of information flow in the system, this boundary affects the circulation to the north of it as well. Once we come up with an additional constraint, we proceed in the way described above until we find a solution without boundary layers for a specified $\psi_{B}$. Our constraint is checked against this solution. For example, if our constraint is in

form of a relation between $\psi_{s}^{(i i)}$ and $T_{s}^{(\text {ii) }}$, we see whether our result fulfills the special relation along the southern boundary. If, as is most likely, it does not, we change our guess for $\psi_{B}$ and start all over again. Using constraints of this type we narrow down the family of possible solutions to a single one.

The same constraint can be worked out in the examples given before, with or without friction in the lower layer. When $r=0$, we guess $\psi_{B}$, solve for the mixed layer, and get the straight characteristics in the interior. These lines intersect the southern boundary where the relation between the temperature and stream function is known. If this relation is not fulfilled, we change $\psi_{B}$ and solve the mixed layer again. 
The procedure is obviously tedious, but enables us to drive a system by conditions on the incoming flow, and replace the arbitrary choice of $\psi_{B}$ (or $\psi_{s}^{(i i)}$ ) with a more sound physical constraint. In general-as is often the case even without constraintswe have to guess at least one boundary condition, and only after a partial solution for the problem for this particular guess, can we get some feedback in order to improve the initial guesses. As an example, we develop such a constraint in form of a relationship between $\psi_{s}^{(\mathrm{ii})}$ and $T_{s}^{(\mathrm{ii})}$.

Let us assume that the large part of the circulation that lies outside our model is connected to our sinking region at $y_{s}$, and to some southern boundary at a distance $\mathcal{L}$ away. In this broad interior we integrate the continuity equation over $y$ while assuming $w=w(z)$, and $v=0$ at the southern boundary, and get $w=\psi_{s}^{(i i)}(z) / \mathcal{L}$. This gives us a relation between the upward velocity in the layered system and the stream function at $y=y_{s}$. If it is further anticipated that the balance in the interior is between vertical diffusion and advection $\left(w T_{z}=\kappa T_{z z}\right)$, we can make use of the result we got for $w$ and solve $\psi_{\mathrm{s}}^{(\mathrm{ii})} T_{z}=\kappa \mathcal{L} T_{z z}$. In particular, this relation holds at $y=y_{s}$, so it is possible to determine either $T_{a}^{(i i)}$ or $\psi_{s}^{(i i)}$, assuming that one of them is known. The solution for the last is immediate, while the one for the temperature, taking it as a a function of depth only, is

$$
T_{s}^{(i i)}(z)=C_{1}+\int_{-D}^{z} d z^{\prime} e^{\left[\int_{-D}^{z^{\prime}} \psi_{\theta}^{(11)}\left(z^{\prime \prime}\right) d z^{\prime \prime}+C_{2}\right]} .
$$

The free coefficients should be determined by the condition that the profile should join smoothly with $T_{n}^{\text {air }}$ along $z=-D$ (because this is the temperature along the northern wall as well as the bottom), and with $T_{s}^{(\mathbf{i})}$ at $\left(y_{s},-h\left(y_{s}\right)\right)$. Thus, we have demonstrated that given an interior dynamic balance, it is sufficient to specify the stream function or the temperature along the southern boundary, and if the interior dynamics is simple enough-as assumed here-the other unknown can be determined. 
A more complicated constraint that couples the upper, lower and interior together can be derived by heat budget considerations. The total amount of heat crossing $z=-h(y)$ in the layered system is $\left(w T+\kappa T_{z}\right) \mathcal{L}$. We already related $w$ to $\psi_{s}^{(\mathrm{ii})}$, and argued that $T=T_{s}^{(i i)}$, but now we can equate this value to the heat flux between the upper and lower regions through the interface, emphasizing the coupling between the solutions in both regions.

\section{Conclusions}

In this section several unique features of the axially symmetric fluid were established. First we showed that conservation of angular momentum and temperature are equivalent to conserving potential vorticity. This is important because no information on the $y$ momentum equation is used, so the fluid can obey the thermal wind balance or not, and still conserve vorticity. Then we went further and proved that if in addition one assumes the thermal wind balance, the fluid trajectories as well as the isotherms are straight lines. It is also apparent that the conservation of angular momentum constrains the north south extent of the vertical gyre. Extending the treatment to a large scale $\beta$ plane approximation or letting the depth of the interface be a strong function of latitude does not change the character of the results.

Without touching upon the conservation of potential vorticity, it is possible to affect the results by adding horizontal $(y)$ and vertical $(z)$ dissipation. The behavior of the new system shows that information flow is propagating downstream and fluid trajectories are no longer straight. These conclusions are not changed when the shape of the interface is altered to suit a different geometry imposed by the forcing.

Solutions to the problem where the value of the stream function along the interface was not specified a priori are made possible by constraining the circulation to merge into a southern domain outside our model. The virtue of this approach is in keeping 
the boundary conditions as physically plausible as possible, so that both the outgoing flow and the heat flux are determined as part of the solution. 


\section{Chapter 5}

\section{Effects of mixing and seasonal cycle on differentially heated flows}

\section{Introduction}

In many references in oceanography it is customary to divide the circulation into several parts, each one depending on different forcing. The part where the surface buoyancy flux determines the circulation and stratification is generally referred to as the thermohaline circulation. It is usually envisioned as an asymmetric vertical cell with cold water sinking in a narrow northern branch, and weak upwelling in the rest of the domain.

The vertical circulation plays an important role in equilibrating the earth climate by transporting the excess heat radiated into the equatorial regions poleward. In this process, atmospheric circulation is also an important factor. More so, there is a relationship between the thermohaline circulation and changes in the global carbon cycle, [Sarmiento and Toggweiler (1984)] while the amount of $\mathrm{CO}_{2}$ in the atmosphere is related to the climatic changes in the earth.

For these reasons, it is important to study the variability of the thermohaline

circulation. Variations on time scale shorter than implied by the conventional $\tau \kappa_{V} / D^{2}$ scale were observed by Brewer et al. (1983), and Roemmich and Wunsch (1984). They reported changes in deep water properties in the North Atlantic over periods of 20 years, but it is not clear how these perturbations affect the surface buoyancy fluxes. Obviously, much larger fluctuations are assumed to have happened in the deglaciation 
periods, for example, Ruddiman and McIntyre (1981), Schnitker (1980), and Boyle and Keigwin (1982).

But these are not the only time scales affecting the circulation. Recent numerical studies by Bryan (1986) suggest that high-latitude small salinity perturbations-as those that probably had happened in glaciation periods-can control deep water formation and interhemispheric circulation, with negative salinity anomaly in one of the hemispheres leading to a fast (order 50 years) decrease in deep water formation at the same hemisphere, and strong asymmetric circulation. Positive perturbation leads to a much slower (order many hundreds of years) asymmetry with weakening of the circulation in the opposite hemisphere resulting in a pole to pole circulation mode.

The thermohaline circulation problem was treated in the past in many analytic and numerical ways, including several laboratory experiments. Some of the work where differential heating was applied over a single horizontal plane, or over two planes separated in the vertical is referenced in the first chapter. To name a few more analytic approaches we can mention the solutions to the thermocline equations (geostrophy with variable stratification and nonlinear heat equation) starting with Robinson and Stommel (1959), and Welander (1959), and reviewed by Veronis (1969); continuing to date with Huang (1984), and Luyten et al. (1983) using layered models.

In this chapter we would like to extend the particular approach dealing with the axially symmetric non-rotating circulation reviewed in the first chapter in two ways. Initially we look at the effects of turbulent mixing in a steady state, thereby applying the ideas developed in the second and third chapters in a model that includes circulation. The same model will then be used to investigate the behavior of the fluid forced by a yearly seasonal cycle. Yearly changes in the ocean are probably one of the more basic features observed, where evolutions that have longer time scales can perhaps be treated as accumulative perturbations of yearly events. 
Circulation in the absence of rotation

For a non-rotating fluid in an annulus, the governing equations are written by substituting $f=0$ in (1.6), resulting in

$$
\begin{aligned}
\delta J(\psi, u) & =\left(\frac{1}{\nu} \frac{\partial^{2}}{\partial y^{2}}+\frac{\partial^{2}}{\partial z^{2}}\right) u \\
\delta J\left(\psi, \nabla^{2} \psi\right) & =R_{a} \lambda^{2} \frac{1}{\delta \sigma} T_{y}+\left(\frac{1}{\nu} \frac{\partial^{2}}{\partial y^{2}}+\frac{\partial^{2}}{\partial z^{2}}\right) \nabla^{2} \psi \\
\delta \sigma J(\psi, T) & =\left(\frac{1}{\kappa} \frac{\partial^{2}}{\partial y^{2}}+\frac{\partial^{2}}{\partial z^{2}}\right) T
\end{aligned}
$$

with $\lambda=D / L$, denoting the aspect ratio, $R_{a} \lambda^{2} / \sigma=\alpha T_{0} / F E^{2}$, and $R_{a}=\frac{\alpha g T_{0} D^{3}}{\nu_{v} \kappa_{v}}$, the Rayleigh number.

Because of the lack of rotation, the $u$ velocity appears in the $x$ momentum equation only. If $u$ vanishes on all boundaries, it is zero everywhere, otherwise, (say $u \neq 0$ at the surface) after the stream function field is found, the zonal velocity is computed by balancing the advection and diffusion in (5.1a).

To simplify the equations further, we make two additional choices. We assume a scale for the stream function $\phi=L \kappa_{v} / D$, and ignore the nonlinear advection term in the vorticity equation. This last assumption turns out to be quite a good one, because the scale for $\phi$ implies that $\delta \sigma=1$, and $\delta=1 / \sigma$ is usually much smaller than the Rayleigh number. ( $\sigma$ is the Prandtl number which for the ocean is usually one or larger.) Previous works cited before all kept this Jacobian term-even though their scaling arguments for the width of the boundary layers did not include it. In the following sections we will show that it is possible to duplicate previous results even when the nonlinear advection in the vorticity equation is neglected. This observation was also acknowledged a posteriori by Beardsley and Festa (1972); their results show that the nonlinear advection terms contribute only about $5 \%$ for Rayleigh numbers 
larger than $10^{5}$. With these simplifications, the equations are reduced to the coupled set

$$
\begin{aligned}
-R_{a} \lambda^{2} T_{y} & =\left(\frac{1}{\nu} \frac{\partial^{2}}{\partial y^{2}}+\frac{\partial^{2}}{\partial z^{2}}\right) \nabla^{2} \psi \\
J(\psi, T) & =\left(\frac{1}{\kappa} \frac{\partial^{2}}{\partial y^{2}}+\frac{\partial^{2}}{\partial z^{2}}\right) T
\end{aligned}
$$

where as before $\nabla^{2}=\left(\lambda^{2} \frac{\partial^{2}}{\partial y^{2}}+\frac{\partial^{2}}{\partial z^{2}}\right)$. The boundary conditions used in solving the equations are no flux through the walls, except at the top where the flux is specified. No other forcing is used to drive the flow, $\psi=0$ along all the walls, and no stress conditions for the velocity components are employed; $\psi_{z z}=0$ along the top and bottom walls and $\psi_{y y}=0$ on the southern and northern walls. These conditions are equivalent to assuming that $\psi=0=\nabla^{2} \psi$ on all walls.

The Rayleigh number was defined before in terms of the horizontal surface temperature gradient. Unlike other works that impose this value, we specify the surface flux, $Q=\rho C_{p} \kappa T_{z}$, so the $\Delta T$ in $R_{a}$ should be replaced by $\frac{Q D}{\rho C_{p}}$, and the Rayleigh number now is defined in terms of flux. The last substitution makes it obvious that changing the heat flux by a factor $c$ is equivalent to multiplying the Rayleigh number by the same factor.

Before we continue exploring these two equations, we review the physical essence of the Rayleigh number. From the hydrostatic balance and the equation of state, the destabilizing gravitational force is $-\alpha g T_{0}$. The opposing viscous convection motion per unit volume $\nu_{v} w_{z z}$ (acceleration) is scaled by $\nu_{v} w / D^{2}=\nu_{v} \kappa_{v} / D^{3}$, where the scale for the vertical motion is deduced from the simplified form of the heat equation $w T_{z}=\kappa_{v} T_{z z}$. With these interpretations, the Rayleigh number is

$$
R_{a}=-\frac{\alpha g T_{0}}{\nu_{v} \kappa_{v} / D^{3}}=\frac{\text { destabilizing gravitational force }}{\text { stabilizing viscous force }}
$$

In our equations, we follow the common procedure of defining the number without the minus sign. This way the dimensionless quantities are positive, and the correct sign is 
retained in the equation. Note also, that it is possible to define a horizontal Rayleigh number with $L, \nu_{H}$, and $\kappa_{H}$ instead of $D, \nu_{V}$, and $\kappa_{V}$. Nonetheless, because of the physical interpretation above, it makes more sense to stick to the definition with the vertical quantities.

For oceanic scales, the Rayleigh number is very large. Typical values of $T_{0}=20^{\circ} \mathrm{C}$, $\alpha g=0.1 \mathrm{~cm} / \mathrm{sec}^{2} /{ }^{\circ} \mathrm{C}, D=5 \cdot 10^{5} \mathrm{~cm}, \nu_{v}=1 \mathrm{~cm}^{2} / \mathrm{sec}=\kappa_{v}$, and $\lambda=10^{-3}$, yield $R_{a}=2.5 \cdot 10^{17}$ and $R_{a} \lambda^{2}=2.5 \cdot 10^{11}$. Nonetheless, because of resolution problems that will be shortly discussed, numerical simulations rarely explore this range of high Rayleigh number. For example, note that the highest value of $R_{a} \lambda^{2}$ used by Beardsley and Festa (1972) was $3 \cdot 10^{5}$ (they used $\lambda=1$ ), whereas several of the laboratory experiments reviewed in their article had $R_{a} \approx 10^{10}$, including Rossby (1965) whose maximum value was $R_{a}=1.6 \cdot 10^{10}$.

\section{Simple analysis of the governing equations}

We know from the temperature and stream function fields [for instance figure (5.2)ab] that the solution to (5.2) has a boundary layer character. There are actually two boundary layers in the solution; one next to the coldest wall (warmest when one heats at the bottom), and one next to the non-insulating wall through which we heat and cool the system. We will refer to these boundary layers as the northern boundary layer or jet, and the thermocline.

The first basic questions about this boundary layer character of the solution are why is this so, where are the layers, why are they occupying that particular location, and what is their typical extent. Because only the last of these questions is treated in the literature, [Rossby (1965), Stern (1975), pp. 216-233] we will bring here a brief answer to these problems.

The existence of the northern boundary layer is a result of the large Rayleigh number. For large values, (5.2a) is approximately $R_{a} \lambda^{2} T_{y}=0$ in the interior, thus, 
$T=T(z)$. This means that the heat equation (5.2b) is now written as $w T_{z}=\kappa_{v} T_{z z}$, yielding $w=w(z)$. If the vertical velocity is independent of latitude, we must have a boundary layer somewhere, because continuity requires that $\int_{y=y_{8}}^{y=y_{n}} w(z) d y=0$ for all $z$. Thus, if everywhere we bring the fluid up, in the boundary layer it must go down, and vice-versa.

The location of the boundary layer and the physical reason behind it are questions that are harder to address. The first usually involves solving for the boundary layer and matching to the interior solution. The procedure usually eliminates all but one of the possible locations of that layer. For example, in this way one finds out that a western and not an eastern boundary layer is the possible solution for Sverdrup flow. The problem with our set is that matching to the interior is not sufficient, and one should match the side wall boundary layer to the thermocline. This is needed because the value of the stream function in the northern jet is by and large determined by its value in the thermocline, since it is this upper flow that turns into the downward jet. Solving analytically for the thermocline is in many respects as hard as solving the full set, because as it turns out from scaling arguments, one should use the full nonlinear heat equation (5.2b). The physics of preferring a northern or southern boundary layer reflects this difficulty. Wave analysis and group velocity arguments are not very fruitful because all waves are suppressed in (5.2). One must conclude that the location is a result of the nonlinear interaction between the flow and the density fields.

In his contribution from (1965) Rossby put forward the proposition that the asymmetry of the circulation is a manifestation of the efficiency of advection as heat transport relative to convection. The interior is being cooled by the downward jet, and is being heated by conduction through one of the boundaries. Because these two effects are of different efficiencies, the thermocline-whose depth is determined by a balance between upward advection and downward diffusion-is shallow, indicating that upward motion 
in the interior is strong enough to force the downward diffusion of heat to be localized next to the non-insulating boundary.

Still, there are some simple arguments to suggest why the downward jet is at the north. The first thing to show is that the interior flow is upward which will lead to a downward jet. The idea behind it is that because diffusion is down-gradient, convection should be upward. Specifically, we already argued that $w=w(z)=\kappa_{v} T_{z z} / T_{z}$. At the bottom of the basin, $T_{z}=0$, (so temperature contours are perpendicular to that boundary) while at the top the flux is specified. If we assume the simplest possible circulation pattern, with no vertical cells, where the temperature is a smooth decreasing function of depth, $\left(T \propto e^{a z}, a>0\right)$ we get $w>0$ in the interior, and as a result there should be a downward jet in the north or south. The southern possibility has to be excluded because the surface temperature at that location is the warmest of all points, and a jet there would have to be maintained against the density gradients. The opposite is true for a northern boundary layer. There, the fluid at the surface is the coldest, and thus can easily sink next to the wall. Although these arguments might not seem very rigorous, they convey the essence of the process, utilizing all the information available in the equations and boundary conditions.

Expressions for the width of the thermocline and the northern jet for the case where $\kappa_{H} \neq 0$, can be found in Rossby (1965), Stern (1975), and Sommerville (1967). The important point in those scaling arguments is that one should solve for the stream function in the thermocline before the northern jet. The scale for the stream function in the thermocline is different than that of the interior, and because it is the fluid in the thermocline that turns into the jet, it is that scale for $\psi$ that should be employed.

The scaling relates the maximum value of the stream function and the thickness of the two boundary layers to the Rayleigh number by a power law. For example, in 
the thermocline, the $z$ coordinate is replaced by a stretched boundary layer coordinate $z=d \xi$ with $d \ll 1$. The scaling balance from (5.2) is

$$
\begin{aligned}
R_{a} \lambda^{2} \Delta T & =\phi d^{-4} \\
\frac{\phi \Delta T}{d} & =\frac{\Delta T}{d^{2}}
\end{aligned}
$$

where $\psi$ and $T$ are re-scaled by $\phi$ and $\Delta T$ to note that in the thermocline they no longer need to have the same scale as in the interior. It is of course possible to use the dimensional balance from equation (1.6), which in the thermocline is written as

$$
\begin{aligned}
& -\alpha g T_{y}=\nu_{v} \psi_{z z z z} \\
& J(\psi, T)=\kappa_{v} T_{z z},
\end{aligned}
$$

and to get the same result as above.

In all previous works the system was forced by imposing an order one horizontal temperature gradient at the top. When driving the circulation by this impressed temperature, the scale for horizontal temperature gradients in the interior is absorbed in the Rayleigh number and $\Delta T=1$ in the above set. This leads to $\phi \propto\left(R_{a} \lambda^{2}\right)^{1 / 5}$, and $d \propto\left(R_{a} \lambda^{2}\right)^{-1 / 5}$. But in this work the flux at the top is specified rather than the temperature. Because this flux is order one, $\Delta T / d=1$ so $\Delta T$ has the same scale as the thermocline depth, and is no longer order unity. The addition of (5.3) now leads to a different power law, $\phi \propto\left(R_{a} \lambda^{2}\right)^{1 / 6}, d \propto\left(R_{a} \lambda^{2}\right)^{-1 / 6}$, and $\Delta T \propto\left(R_{a} \lambda^{2}\right)^{-1 / 6}$.

In the northern jet a set similar to (5.3) is valid

$$
\begin{aligned}
R_{a} \lambda^{2} \frac{\Delta T}{\ell} & =\frac{\phi}{\ell^{4}} \lambda^{2} \\
\frac{\phi \Delta T}{\ell} & =\frac{\Delta T}{\ell^{2}}
\end{aligned}
$$

with $\ell$ as the horizontal extent of the layer. The scale $\Delta T$ for the temperature in the downward jet is still unknown, but that for the stream function $\phi$ is the same as the one derived for the thermocline because it is the same flow that turn into the downward jet. It turns out that the width of the layer is the same as the depth of the thermocline 
$\ell \propto\left(R_{a} \lambda^{2}\right)^{-1 / 6}$, so the transports in the two boundary layers are equal. The scale for $\Delta T$ in the jet is determined from (5.4a) to be $\Delta T \propto\left(R_{a} \lambda^{-4}\right)^{-1 / 3}$, compared to $\Delta T \propto\left(R_{a} \lambda^{2}\right)^{-1 / 6}$ for the full basin width. For the typical values assumed before, the temperature gradient in the northern jet is very small, $1.3 \cdot 10^{-8}$ compared to the interior temperature changes, indicating that there is no thermal boundary layer along the northern wall.

We conclude, that when the Rayleigh number is given in terms of flux, the horizontal temperature gradient is an external parameter, and the boundary layer is deeper while the circulation is weaker for the same value of $R_{a}$. Our numerical results will be in agreement with this power law.

These scales above are the reason why it is hard to realize numerically a circulation with large $\left(R_{a} \lambda^{2}\right)$, say $10^{12}$. For a case like that, the boundary width is $1 / 100$ of the extent of the basin when the upper temperature is specified, so using conventional grid approximations will lead to large grids and prohibitive amount of computer time.

These results can also be verified while looking at the conservation of heat in the system. Equation (5.2b) is integrated over an area $d A^{*}$ bounded between the top and bottom surfaces, the southern boundary, and in the north by a latitude line $y=y^{*}$.

$$
\int[\vec{u} \cdot \nabla T-\nabla(\kappa \nabla T)] d A^{*}=\oint \vec{u} T \cdot \hat{n} d \ell-\oint \kappa \nabla T \cdot \hat{n} d \ell
$$

with the conventional notation of $\vec{u}=(v, w), \hat{n}$ the outward normal unit vector, and $d \ell$ a perimeter element. The only integration boundary that is not rigid is at our arbitrary latitude $y^{*}$, and the only non-insulating wall through which the net temperature gradient does not vanish is the top one. With these observations the integral above collapses to

$$
\int_{-1}^{0} v T d z=\int_{0}^{y^{*}} Q(y) d y
$$

where the domain varies in the vertical between $z=-1$ (bottom) and $z=0$ (top) and $Q(y)$ is the specified flux at the top. It is useful to split the temperature into 
two $T=T^{*}+\bar{T}$, where $\bar{T}(y)$ is the vertically average temperature in any latitudinal cross section. This depth independent part of the temperature does not contribute to the heat transport because $\bar{T} \int_{-1}^{0} v d z=0$. The integral on the 1.h.s. of (5.5) has two contributions; one from the heat transport in the interior, and one from that in the thermocline:

$$
\int_{-1}^{0} v(T-\bar{T}) d z=\int_{-1}^{d} v(T-\bar{T}) d z+\int_{d}^{0} v(T-\bar{T}) d z
$$

where the asterisks were dropped. To show that the contribution of the second is much larger than the first, it is convenient to write two approximate conservation statements

$$
\begin{array}{r}
d v_{b}+(1-d) v_{i}=0 \\
d\left(T_{b}-\bar{T}\right)+(1-d)\left(T_{i}-\bar{T}\right)=0
\end{array}
$$

with the subscripts $b$ and $i$ for boundary (thermocline) and interior values. The first equation above states that the mass transport through any latitude line is zero, while the second represent the fact that the total heat content in the column is fixed, and is equal to $\bar{T}(y)$ at any given latitude. When the values of $v_{i}$ and $T_{i}$ are substituted in (5.6) the result is

$$
\int_{-1}^{0} v(T-\bar{T}) d z=d v_{b}\left(T_{b}-\bar{T}\right)\left(1+\frac{d}{1-d}\right) \approx d v_{b}\left(T_{b}-\bar{T}\right)
$$

which is the contribution from the transport in the thermocline [the second integral on the r.h.s. of (5.6)] when $d$ is small, or equivalently, when the Rayleigh number is large. Using the scales found before $\left[v=\phi / d \propto\left(R_{a} \lambda^{2}\right)^{1 / 3}, d \propto\left(R_{a} \lambda^{2}\right)^{-1 / 6}\right.$, and $\Delta T \propto\left(R_{a} \lambda^{2}\right)^{-1 / 6}$ ] the 1.h.s. of (5.5) is order one, as it has to be in order to balance the flux on the r.h.s.

Different possible balances than those of (5.3)-(5.4) are also possible. For instance, in the absence of horizontal temperature diffusion $\left(\kappa_{H}=0\right)$ the two terms in the Jacobian in (5.2b) balance each other. If the top temperature is specified, it turns out 
that $d \propto R_{a}^{-1 / 5}$ while $\ell \propto R_{a}^{-4 / 15}$, resulting in a northern jet that is thinner than the thermocline.

It is important to note that in the limit of a small aspect ratio, (when the horizontal diffusion is weak or even completely absent) there is a thermocline structure whose balance is the same as with large $\kappa_{H}$. This is in contrast to the analytic solution derived in chapter 2 when no flow field was present. Our results showed that the temperature field was depth independent if the fluid was isotropic or when only acrossisopycnal mixing was assumed in density coordinates. The conclusion is of course that the circulation is a crucial dynamical constraint in determining the final shape of the isotherms. In particular, we know that the thermocline structure is determined from upward advection and downward diffusion, a balance that can still be maintained in the small aspect ratio limit.

The scaling argument shows that the horizontal oceanic temperature gradient, which is $\Delta T R_{a}$ is proportional to $R_{a}^{5 / 5}$. On the other hand, the heat flux is proportional to $R_{a}$. Thus, if the flux is represented as $\kappa T_{z} \propto T-T^{\text {air }}$ the orders of magnitude above suggests that for large Rayleigh numbers one could possibly approximate the heat flux by $\kappa T_{z} \propto T^{\text {air. }}$. This is equivalent to specifying the flux with no knowledge of the sea surface temperature. However, it is not clear that even if $\left\|\kappa T_{z}\right\|>\|T\|$, the possibility of $T=T^{\text {air }}$ is not a better approximation than $\kappa T_{z} \propto T^{\text {air }}$. In any case, it is important to note that as the Rayleigh number increases the flow becomes more and more rapid, and the nondimensional oceanic surface temperature gradient decreases. This would have an effect on flux conditions for atmosphere-ocean models. 


\section{Parameter range}

One of the underlying assumptions in (1.6), and hence in (5.2) is that the viscosity and thermal conductivity used in parameterizing the stress terms are independent of location, so a term like $\nabla(\kappa \nabla T)$ was approximated by $\kappa \nabla^{2} T$. When the idea of convective overturning was introduced in chapter 2 , this assumption was broken, and we let $\kappa$ depend on the local stratification (2.4). The same method is used here so the new equations are written

$$
\begin{aligned}
-R_{a} \lambda^{2} T_{y} & =\nabla^{4} \psi \\
J(\psi, T) & =\nabla(\kappa \nabla T) .
\end{aligned}
$$

[It is possible to write equations (1.6) with $\kappa$ and $\nu$ in front of the $\frac{\partial^{2}}{\partial z^{2}}$ derivative and not $\frac{\partial^{2}}{\partial y^{2}}$. Apart from this change the equations will look the same if we redefine $E=\frac{\nu_{H}}{f_{0} L^{2}}$, $\sigma=\frac{\nu_{H}}{\kappa_{H}}$, and $\left.\delta=\frac{\phi L}{\nu_{H} D}\right]$. The distinction between the two cases investigated in this work can be made using the diffusivity $\kappa$.

\section{Standard case, no mixing}

All the works cited in the introduction assumed that in (5.7) $\nu=1=\kappa$ everywhere, so that $\sigma$ is fixed. This of course allows the final solution to include regions where the stratification is unstable. We refer to this case study as the standard, or the no mixing case, and use it as a reference with which our results are compared. We adopt the notation where the values of any parameter in the stable and unstable regions are given as two numbers inside brackets, so for this standard case $\kappa=\sigma=(1,1)$. 


\section{Mixing case}

As implied by the name, the assumption is that $\kappa$ depends on the local vertical gradient of $T$, thus, when the stratification is unstable the density field is adjusted. The value used was $\kappa=(1,100)$ so $\sigma=(1,1 / 100)$.

When mixing is present the effective Rayleigh number is no longer constant but varies locally, because it depends on $\kappa_{v}$. For example, if we use the previous values of $\kappa_{v}=1=\nu_{v}$ and we increase them a hundred fold in the unstable regions; then $R_{a}=\left(R_{a}^{\prime}, R_{a}^{\prime}\right)$ in the standard case, and $R_{a}=\left(R_{a}^{\prime}, R_{a}^{\prime} / 100\right)$ when density is mixed.

\section{Forcing}

Three types of surface heat fluxes are used to drive the circulation in the domain, normalized to a box with a unit dimension in each direction. The aspect ratio $\lambda$ is equal to one throughout this chapter. The same value was also used in other works, like Beardsley and Festa (1972). It is usually absorbed in the Rayleigh number, and its only influence will be through the operator on the right hand side of (5.2a), having small effects on the flow field.

1) A forcing that is fixed in time and taken as the canonical cosine. With this boundary condition the net flux into the system is zero at any given time.

2) A seasonal forcing that varies with time, but has a yearly cycle through which the net flux vanishes. This forcing is represented by a cosine whose amplitude is shifted four times a year to represent the four seasons. The values that are added to $\cos (\pi y)$ are $0.80,-0.19,-0.75$, and 0.14 corresponding to summer, autumn, winter, and spring respectively. The reason that the seasons are only almost symmetric, and the maximum amplitude in the summer is not the negative of the winter is to indicate that although the yearly net input is zero, the heating in the summer is a touch stronger than the cooling in the winter [Oort and Vonder Haar (1976)]. For this time dependent case, the parameter $\tau \kappa_{v} / D^{2}=0.02$ when 
$\kappa_{v}=1$. The time step is small enough to ensure hundreds of steps per season, so the cycle is very well resolved.

The results will be presented at the end of each season, so a figure that will be labeled summer, is actually a realization right before autumn conditions prevail.

3) Surface flux that is fixed in time but corresponds to permanent cooling or heating. Two heat fluxes are employed from the values given above; continuous summer conditions, and continuous winter conditions. It will be shown that even though the total heat content of the system is time dependent, the flow field and the stratification reaches a steady state.

Note, that the value specified for the heat flux is $\kappa_{v} T_{z}$ with $\kappa_{v}$ as the vertical diffusivity in the stably stratified region. This way the flux is not increased a hundred fold at the unstable regions where and when $\kappa_{v}$ increases. 


\section{Numerical scheme}

Equations (5.7) were solved numerically using a regular uniform finite difference grid with a resolution of $17 \times 17$ in $y$ and $z$. This turns out to be sufficient in resolving the boundary layer character of the solution even for our highest Rayleigh number of $2 \cdot 10^{5}$. The diffusive terms in the heat equation were approximated using a second order finite difference given in (2.7). The Jacobian term was estimated by the Arakawa scheme [Arakawa (1966)]. Detailed information on this approximation in the interior and along all the boundaries is presented in appendix A. Given a stream function field, (5.7b) was stepped forward in time to find the new temperature field

$$
T^{(n+1)}=T^{(n)}+\Delta t\left[\frac{1}{\kappa} T_{y y}^{(n)}+T_{z z}^{(n)}-J\left(\psi^{(n)}, T^{(n)}\right)\right]
$$

where $\Delta t$ is the time step, and $A^{(n)}$ corresponds to evaluating $A$ at time $n \Delta t$. For this equation only, the derivatives and the Jacobian notation stands for the finite difference approximation explained before. The time step can be computed using the Von Neumann stability as explained in the section devoted to numerical implementation in the second chapter. It is the same as (2.8) but with an added term in the denominator to account for the advection of the density field; thus, it is always smaller than the purely diffusive limit. The extra term when evaluating the Jacobian in the Arakawa scheme

is simply $\frac{\psi_{\max }}{\Delta y \Delta z}$, with $\Delta y$ and $\Delta z$ as the grid spacing, and $\psi_{\max }$ is the maximum value of the stream function.

Once the temperature field is known, the 1.h.s. of (5.7a) is evaluated, and treated as forcing for the Laplace equation. It is more convenient numerically to solve for a $\nabla^{2}$ operator instead of $\nabla^{4}$ one, hence the last equation is written as the set

$$
\begin{array}{r}
\Psi=\nabla^{2} \psi \\
R_{a} \lambda^{2} T_{y}=\nabla^{2} \Psi
\end{array}
$$

with the boundary conditions as explained before; $\psi_{z z}=0$ along the top and bottom boundaries, and $\psi_{y y}=0$ along the side walls. These boundary conditions follow 
immediately from our assumption that $\psi=0=\Psi$ on all boundaries. The scheme for solving each of equation (5.9) follows Buneman (1969). Once $\Psi$ is known everywhere from (5.9b), we use the same Poisson solver again to get $\psi$ from (5.9a). The new stream function field is used in (5.8) to evaluate the Jacobian needed for the new temperature. This new temperature is now employed in (5.9b) and the procedure is repeated until a steady state is reached.

\section{Results}

Equations (5.7) were solved numerically for the parameter range described before, and insulating boundaries except for the specified heat flux at the top. Steady state or periodic steady state results for Rayleigh numbers of $2 \cdot 10^{4}$ and $2 \cdot 10^{5}$ are presented. Where appropriate, contour plots are included to visualize information on the temperature $(T)$, stream function $(\psi)$, thermocline $\left(T_{z}\right)$, and forcing $\left(T_{y}\right)$. For these figures the abscissa and the ordinate are latitude and depth. At other instances, the development of the maximum value of the stream function $\left(\psi_{\max }\right)$ is traced with time. The heavy line on the contouring marks the difference between the region where $T_{z}>0$ (to its left) and that with $T_{z} \leq 0$. 


\section{Constant heat flux}

\section{No mixing}

In this reference case represented in figures (5.1) a-c and (5.2) a-c, one can detect the usual features reported in other works, including the strong dependence on the Rayleigh number in the intensification of the northern boundary layer, the shallowing of the thermocline and the increase in value of $\psi_{\max }$. As in Beardsley and Festa (1972), the power law that relates $\psi_{\max }$ to $R_{a}$ converges slowly to the desired value which for our case is $1 / 6$ and not $1 / 5$. The transition from proportionality (for very small Rayleigh numbers not documented here) to a power law in the relationship between $\psi_{\max }$ and $R_{a}$ is smooth but slow. With increasing $R_{\alpha}$, the eye of the gyre moves towards the top northern corner of the domain. Because $\psi=c R_{a}^{-1 / 6}$ where $c$ is unknown, it is possible to check the power law by evaluating $\frac{\psi_{\max }\left(R_{a}^{1}\right)}{\psi_{\max }\left(R_{a}^{2}\right)}=\left(\frac{R_{a}^{1}}{R_{a}^{2}}\right)^{\alpha}$ where $\alpha$ is to be determined. The values of $\psi_{\max }$ at our disposal are $3.56,6.05,7.37$, and 8.05 for the Rayleigh numbers of $2 \cdot 10^{4}, 1 \cdot 10^{5}, 2 \cdot 10^{5}$, and $3 \cdot 10^{5}$ (the $1 \cdot 10^{5}$ and $3 \cdot 10^{5}$ are not represented in the figures). These numbers lead to $\alpha=0.33$ for the first two Rayleigh numbers, $\alpha=0.28$ from the third and second, and $\alpha=0.22$ from the last two. The convergence to $1 / 6$ is rather slow. For example, Beardsley and Festa (1972) reported that in their experiments a value of 0.36 was reached for the higher Rayleigh numbers, where the final value should be $1 / 5$. 

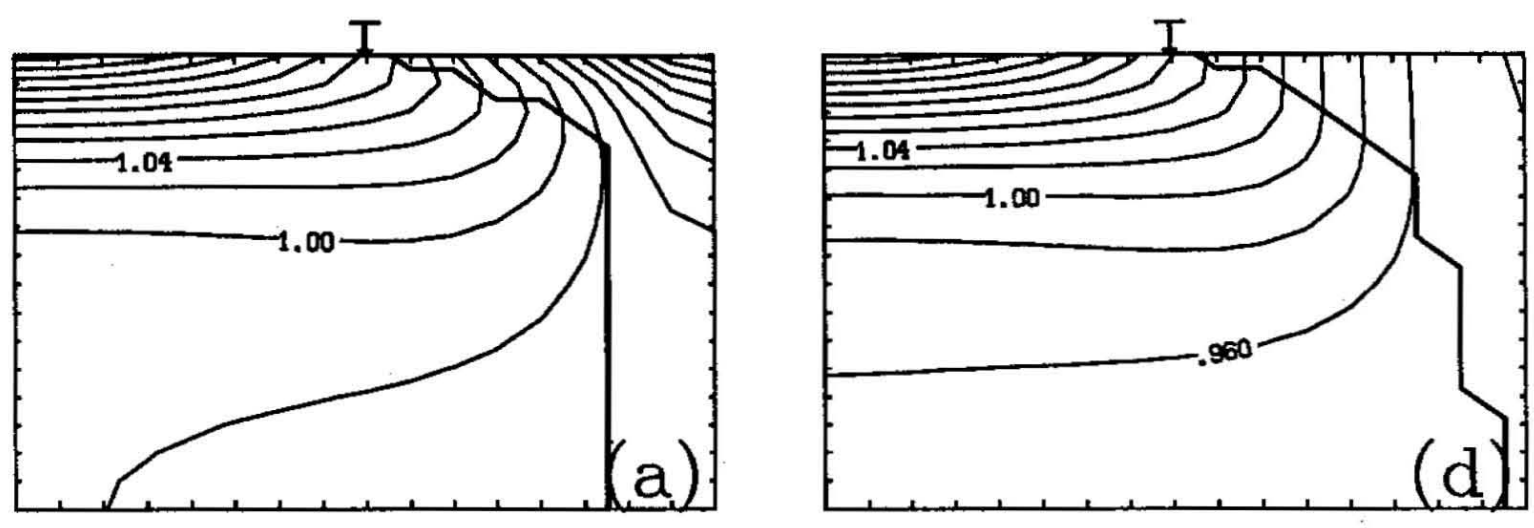

$\psi$
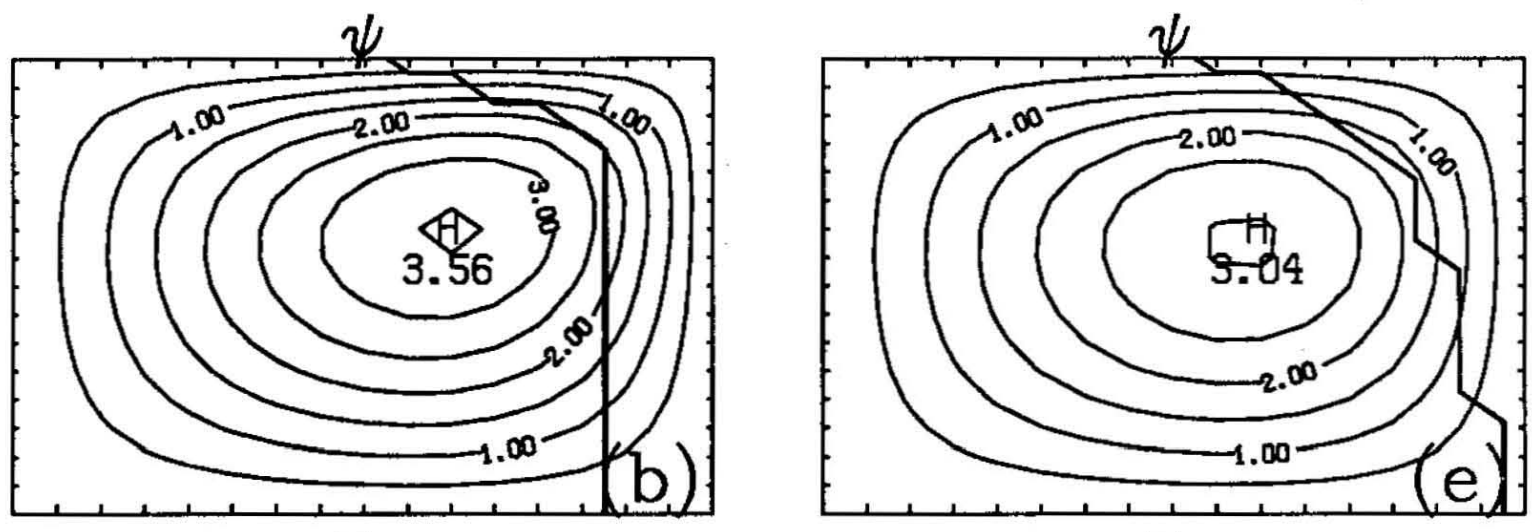

$\mathrm{T}_{\mathrm{z}}$

$\mathrm{T}_{2}$
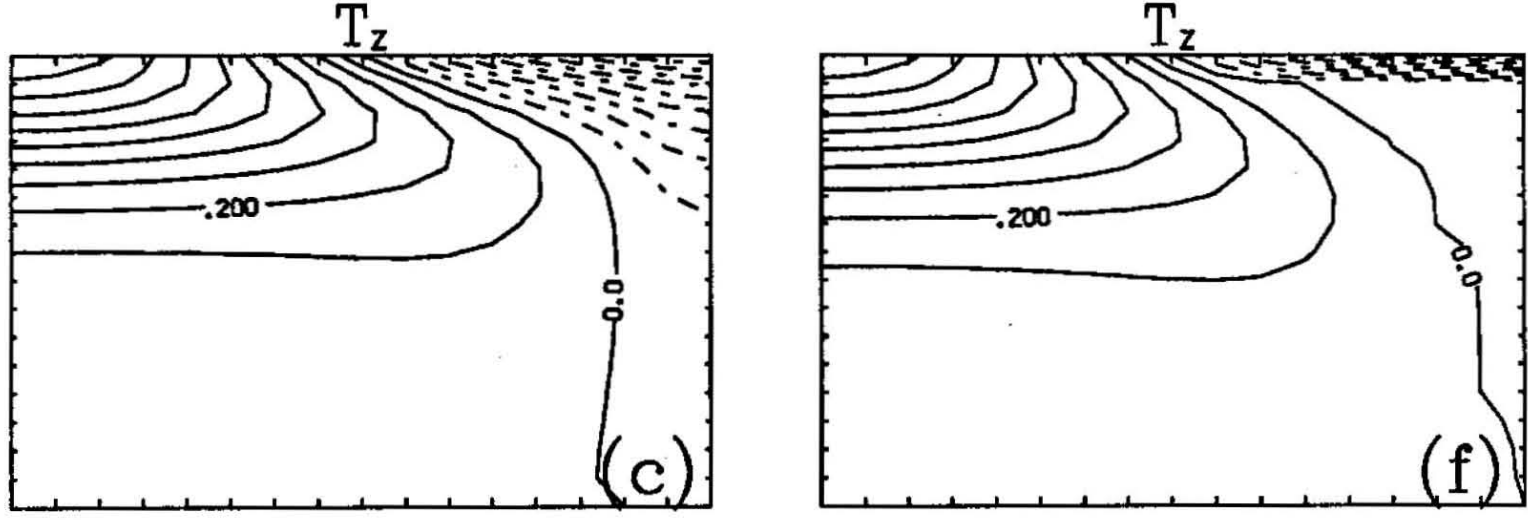

Figure (5.1). The steady state for cosine heat flux and $R_{a}=2 \cdot 10^{4}$. Standard case shown in the left column, density mixing in the right. (a), (d) $T$. (b), (e) $\psi$. (c), (f) $T_{z}$. 

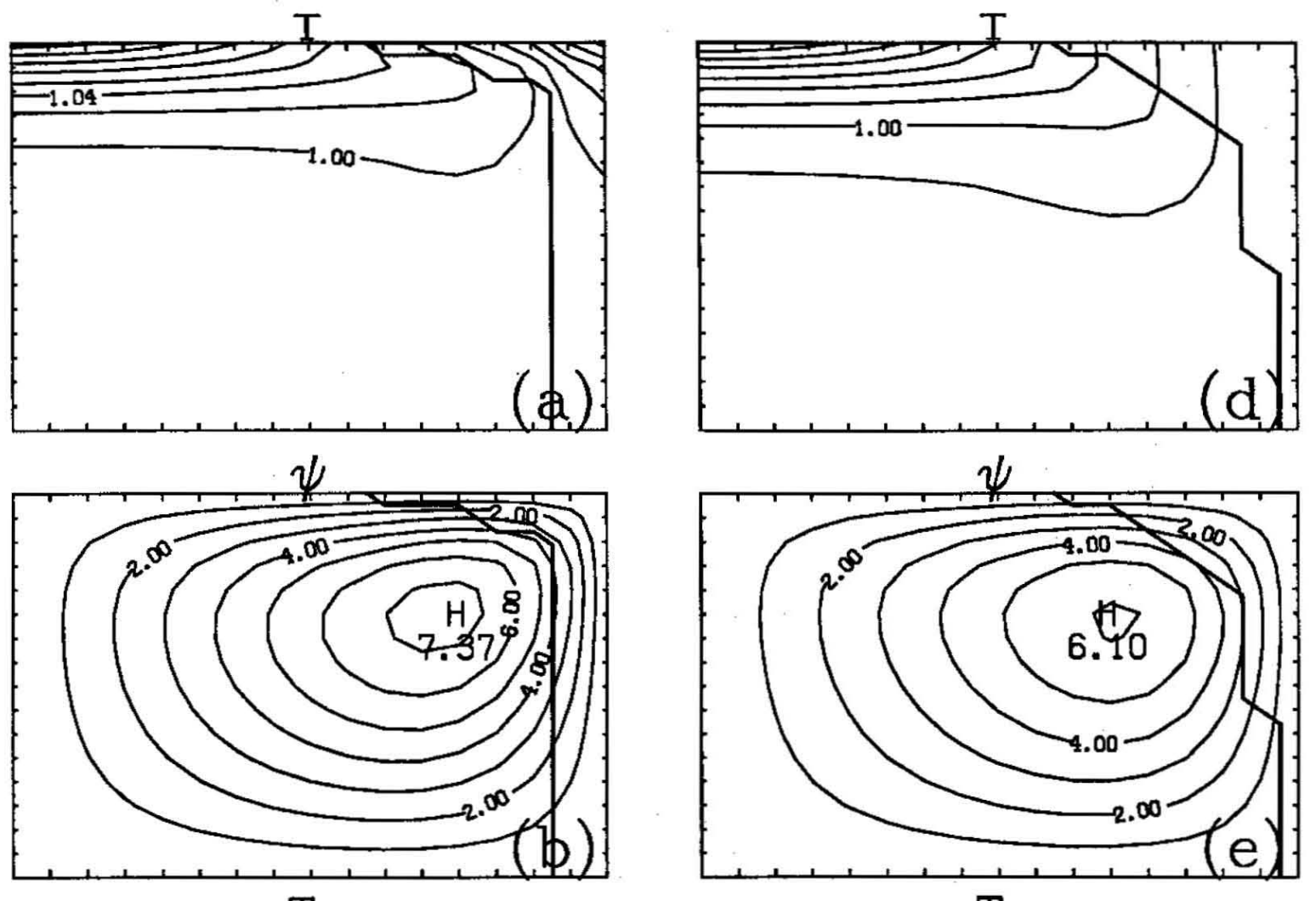

$\mathrm{T}_{7}$

$\mathrm{T}_{7}$
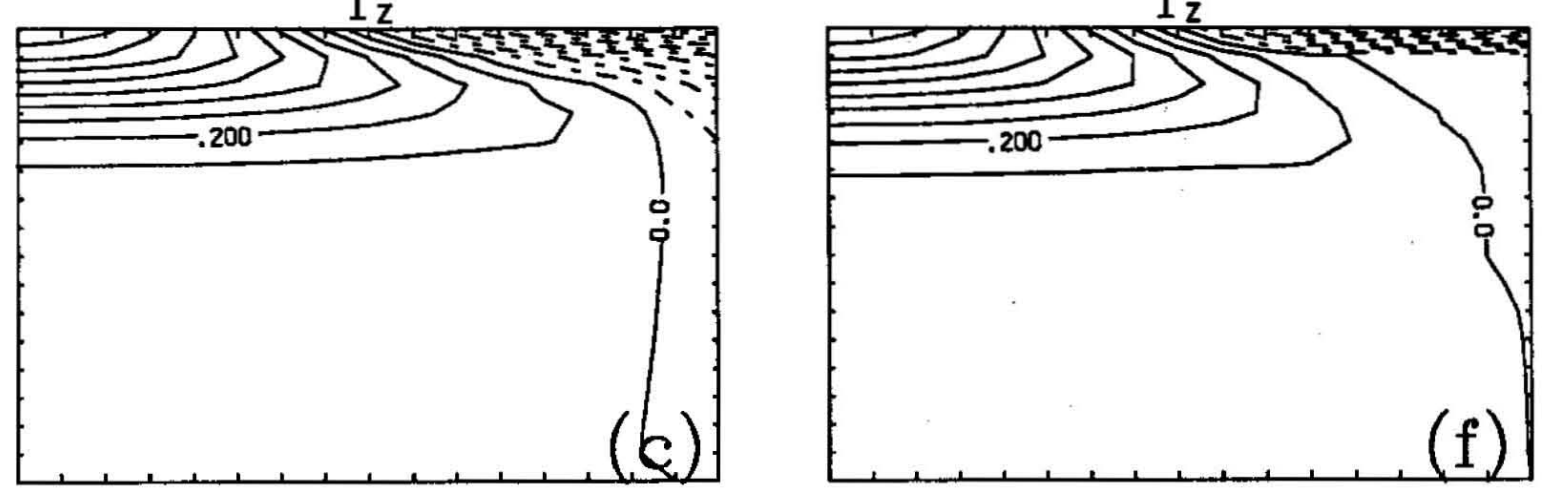

Figure (5.2). The same as (5.1) but for $R_{a}=2 \cdot 10^{5}$. 


\section{Mixing}

Compared to the non-mixing case, $\psi_{\max }$ decreases as presented in figures (5.1)d-f and (5.2) $d-f$, (because the effective Rayleigh number is now smaller in the unstable region) but the temperature is warmer next to the surface. Compare for instance the 1.00 contour in figure (5.1) a to (5.1)c, and in figure (5.2)a to (5.2)c. At first glance this would suggest that although the flow field is weaker, the thermocline is shallower. This opposes the hypothesis that the thermocline depth is determined by and large by a balance between upward flow and downward diffusion $\left(w T_{z}=\kappa T_{z z}\right)$; resulting in a deeper thermocline when the flow field weakens. Here, the $T_{z}$ contours in figures (5.1) a,f,i and (5.2)a,f,i prove very useful, and show that actually the thermocline is now deeper as one would expect. When there is convective overturning, the interior fluid is colder because of vigorous vertical mixing in the northern regions. But since the total amount of heat in the system is fixed, the surface temperature in the southern region is higher, a phenomenon not connected to the thermocline depth.

The shape of the interface changed with respect to the standard case. It is more curved and does not go north next to the surface and then straight down, because the overturning is effective in bringing cold water down. 


\section{Discussion}

Whether there is mixing or not, the geometry of the cell becomes successively asymmetric as the Rayleigh number increases. The center of the cell (where $\psi=\psi_{\max }$ ) moves towards the top north corner with increase in $R_{a}$. The deep fluid is nearly isothermal, and a distinct thermocline feature, where the isotherms are closely packed, is observed next to the top non-insulating boundary.

These features are related to the increase in the importance of the heat convection as the Rayleigh number increases. Heat is advected downward in the northern boundary layer, and upward and to the south in the weakly stratified interior. The conduction is important in the thermocline region where diffusion heats and cools the upper layer. The description above is examined in the next section where we show that to a large extent, $w T_{z}=\kappa T_{z z}$ is a very good approximation to (5.2b) over most of the domain, with the exception of the northern jet where the $T_{y y}$ term is important.

The mixing process makes the effective Rayleigh number a local variable, depending on the stratification. The main patterns discussed above are kept intact whether there is mixing or not, but when there is, the fluid gets colder, the overturning region broader, and the circulation less intense. In this respect the mixing is very helpful when dealing with realistically large Rayleigh numbers, because its moderating effect helps in reducing the otherwise very intense boundary layer character of the circulation.

It is important to note that even if we were to increase the turbulent mixing by a value which is different than our choice of a hundred $[$ say, $\kappa=(1,1000)$ instead of $\kappa=(1,100)]$ the final result will not be affected. In the second chapter it was proved that the solution in the unstable region is to first order independent of $\kappa$. The same result holds here (and was also verified by a set of numerical experiments) even though the effective Rayleigh number depends on this parameter.

The horizontal surface temperature gradient is sensitive to the mixing and the Rayleigh number. By scaling argument we showed that were the surface heat flux 
is proportional to $R_{a}$, the horizontal surface temperature gradient, $R_{a} \Delta T$, decreases with increasing Rayleigh number, following a $R_{a}^{5 / 6}$ power law. This result is verified by drawing $[T(1)-T(0)] R_{a}$ versus $R_{a}$ on a $\log -\log$ scale. It turns out that indeed one gets a straight line with a slope of $5 / 6$, which is in agreement to the theory. The values we get for $\Delta T$ are $0.376,0.286,0.254$, and 0.238 when the Rayleigh numbers are $2 \cdot 10^{4}$, $1 \cdot 10^{5}, 2 \cdot 10^{5}$ and $3 \cdot 10^{5}$ respectively. When turbulent mixing is parameterized, the corresponding values are 0.258 , and 0.177 for $R_{a}=2 \cdot 10^{4}, 2 \cdot 10^{5}$, both lying on the same $5 / 6$ slope. It is apparent that the dimensionless $\Delta T$ decreases with the Rayleigh number, and that it is smaller when mixing is present. The fact that we get the same slope with or without mixing might indicate that the power law is the same but the proportionality constant is different. For modeling purposes, even if the total gradient is unchanged, the local air to sea temperature gradient is different in the two cases, and so might be the heat flux.

In Summary, compared with the standard case, mixing has the following effects.

- The intensity of the circulation is reduced, the thermocline is deeper and the northern jet is wider, while the center of the gyre moves down and to the north.

- The interior of the fluid is colder, and the isotherms are more crowded next to the surface.

- The temperature structure in the cooled region is vertical next to the surface.

- The stream function contours are more rounded and the transition from a northward flow in the thermocline to the downward jet is less sharp.

- In both cases the horizontal sea surface temperature gradients decrease with the Rayleigh number following the same power law. 


\section{The heat equation and its leading order balance}

The numerical solutions of equations (5.7) presented in this chapter show that the interior fluid is to a large extent isothermal. The circulation is characterized by two boundary layers; a thermocline where the flow is northward, and a downward jet next to the coldest wall. The interior fluid has an upwelling motion everywhere.

The magnitudes of the various terms in the heat equation are presented in figures (5.3)a-d, where $v T_{y}, w T_{z}, T_{y y}$, and $\kappa T_{z z}(\kappa=1)$ are plotted as a function of depth at different latitudes. The appropriate dash pattern presenting each of the terms is marked on the figures by the characters a-d, corresponding to $v T_{y}, w T_{z}, T_{y y}$, and $\kappa T_{z z}$. The profiles in the four figures (5.3)a-d are taken at latitudes $2 / 16,5 / 16,8 / 16$, and $11 / 16$ respectively, where latitude 0 corresponds to the southern wall, and 1 to the northern extent of the domain. For this particular simulation with $R_{a}=2 \cdot 10^{5}$, latitude $13 / 16$ is the approximate boundary between the downward jet and the rest of the fluid. The vertical axis of each plot is depth, and varies from 0 at the bottom to 16 at the surface. The inner plot in each figure shows the full variations of each term top to bottom, while the larger outer plot is an enlargement of the region below the thermocline.

It is apparent that near the bottom all the terms are small, while the balance in the thermocline is very different, with large velocities and spatial gradients. In the region below the thermocline, the main balance is clearly between vertical advection $w T_{z}$, and vertical diffusion $\kappa T_{z z}$, with small horizontal advection and diffusion. At the northernmost latitude presented in figure (5.3)d, because of the vicinity to the northern jet, we start picking up contributions from the $v T_{y}$ and $T_{y y}$ terms.

The same set of equations analyzed here was also studied by Roberts (1977) in the limit of infinite Rayleigh number. The problem addressed by Roberts is that of an infinite quadrant whose walls are insulating, with the exception of a small strip near its vertex where heating (or cooling) is applied. In terms of our $y-z$ coordinate system 

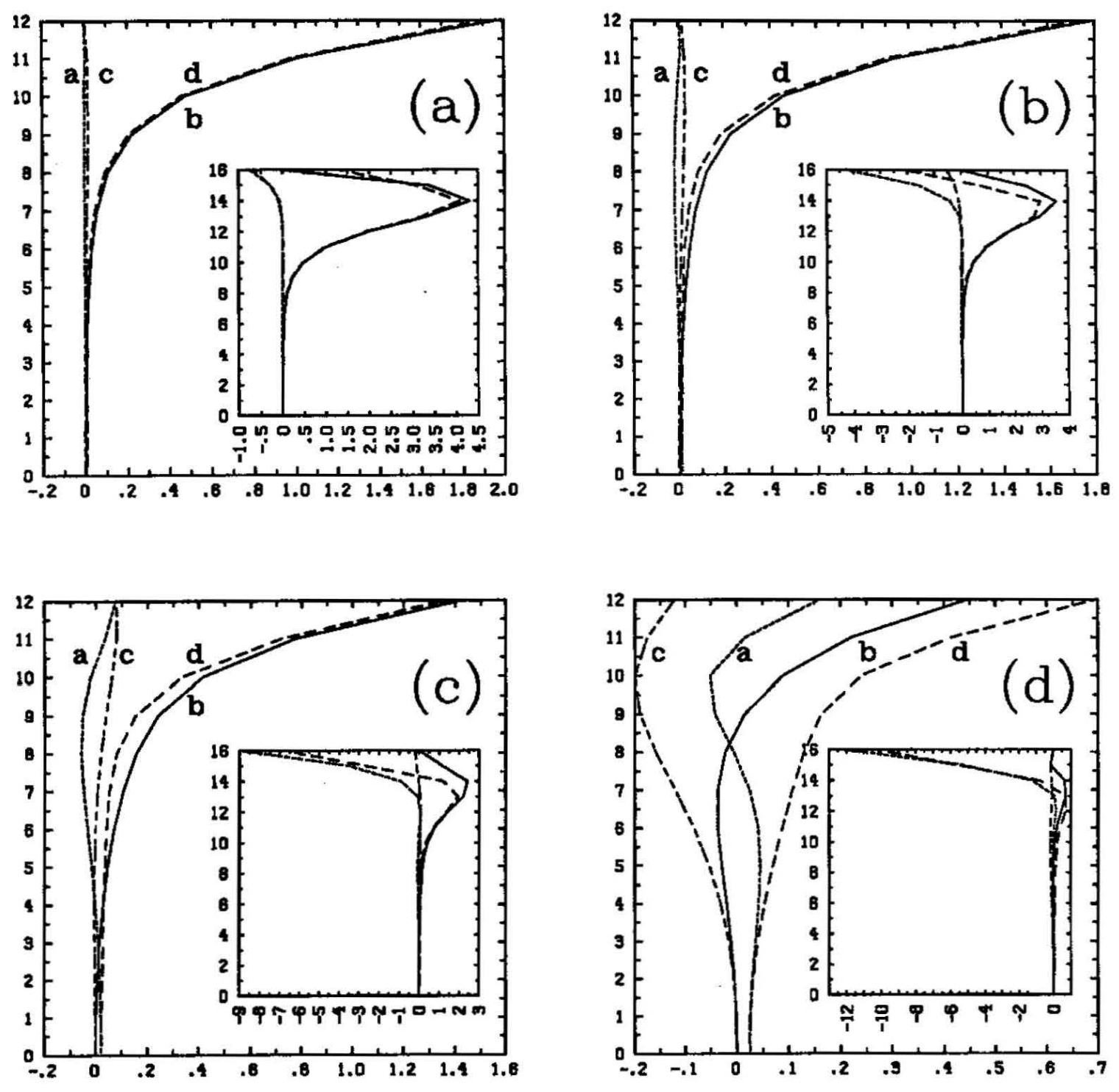

Figure (5.3). The variations with depth of the terms in the heat equation (5.7b). Line (a), $v T_{y}$; line (b), $w T_{z}$; line (c), $T_{y y}$; line (d), $\kappa T_{z z},(\kappa=1)$. The profiles are taken at four different latitudes with the inserted figure showing the full variation with depth, while the outer one corresponding to the fluid below the thermocline. The Rayleigh number is $2 \cdot 10^{5}$, and the south-north latitude values are normalized between 0 and 1. (a) Section computed at latitude $1 / 16$. (b) Latitude $5 / 16$. (c) Latitude $8 / 16$. (d) Latitude 11/16. 
we can think of a quarter plane with its vertex at $(y, z)=(0,0)$, extending to $(y, z)=$ $(-\infty,-\infty)$, and being cooled from the top along a small strip from $(y, z)=(0,0)$ to $(y, z)=(-l, 0)$.

Following Roberts, the buoyancy loss will cause fast viscous convection in narrow boundary layers, one next to the $z=0$ wall, and the other right by the $y=0$ boundary. The interior fluid is isothermal. The mathematical justification is as follows. In the limit of $R_{a} \rightarrow \infty, \psi$ will be very large from (5.7a), thus, the zero order balance in the heat equation (5.7b) is $J(\psi, T)=0$, leading to $T=f(\psi)$. The value of $f(\psi)$ is determined from the small diffusive correction. The equation is written as $\nabla \cdot(\vec{u} T-\kappa \nabla T)=0$, and integrated using Gauss's theorem, $\int_{A}\left(\vec{u} \cdot \hat{n} T-\frac{\partial T}{\partial n}\right) d A=0$, with $A$ representing an area, and $n$ as the outward normal. If the area is closed by a stream line, the first integral vanishes, thus, replacing $\partial T / \partial n$ by $f_{\psi} \psi_{n}$ leads to $f_{\psi}=0$ implying that $f(\psi)$ is a constant, so the interior is isothermal.

The same arguments can be extended to our finite domain and would apply if there are closed stream lines in the interior that do not pass through the boundary layers, and if the thermal structure were to exhibit a strong boundary layer character with narrow boundary layers next to the four walls. But it is evident that below the thermocline our results are not in agreement with the balance proposed by Roberts, where to a first approximation $J(\psi, T)=0$.

One can argue that the root of the discrepancy is our finite Rayleigh number. But this is probably not the case, because Roberts suggests that a Rayleigh number as small as 100 would be a reasonable lower bound for his arguments, while in our experiments the Rayleigh number was much higher. Moreover, our quantitative results concerning the width of the boundary layers are different than his. Roberts predicts a $R_{a}^{-1 / 3}$ thickness law, whereas our solutions are much closer to the prediction of a $R_{a}^{-1 / 6}$ power law when the flux is specified, and a $R_{a}^{-1 / 5}$ dependence when the temperature 
is given. In addition, all our stream lines seems to pass through the boundary layers and no closed stream lines are observed in the interior.

The strong boundary flow proposed by Roberts is a result of his assumption that as $R_{a} \rightarrow \infty$, so does the stream function. It seems reasonable to assume that the velocity would increase, because the density gradients are scaled by the Rayleigh number in the $z$ momentum equation, so one needs to have large $\nabla^{2} w$ to balance them. But this does not necessarily mean that $\psi$ is infinitely large, but only that its gradients are strong, as is the case in the boundary layers (the expression relating $\psi_{\max }$ to $R_{a}$ is different in our work and that of Roberts). In the interior we would argue that as $R_{a} \rightarrow \infty$, $T_{y} \rightarrow 0$, so $\psi$ is still small enough so that in the heat equation, a term like $v T_{y}$ can be smaller than $T_{z z}$.

The contrast between our model and that of Roberts can be visualized when dealing with Bénard convection. In that case, the circulation is confined into a cell that is differentially heated along the top and bottom. It now seems reasonable that the motion will be in the boundary layers surrounding the cell. Following a fluid parcel starting at the top left corner, the parcel is cooled until it reaches the right wall where it sinks without temperature changes. Along the bottom boundary the fluid warms up, and retrieves its initial temperature when reaching the bottom left corner. From that point it advects upward next to the insulating left boundary, reaching the top left corner and starting another cycle. Our problem is quite different from the Bénard convection because the differential heating is applied next to a single wall only. This means that a parcel leaving the top north corner has to warm up while moving along the insulating boundaries until it reaches the temperature of the top south corner. This can perhaps be done if there are thermal boundary layers next to the walls, and the heat is diffused from the interior to those layers. Because of the different nature of the two problems, it is not very clear that even if one adopts Roberts heating configuration in our finite domain-by fixing the temperature along most of our upper boundary, 
and applying local heating next to the northern corner-his results will be reproduced numerically.

The previous arguments in this section neglect a fundamental difference between our system and that of Roberts-and for that matter other works like Beardsley and Festa (1972), and Sommerville (1967). In those works the surface temperature is specified, so the l.h.s. of equation (5.7a), which is proportional to $T_{y}$, is given along the surface. On the other hand, the forcing, which is the actual heat flux, can only be calculated once the solution is known. Our analysis is different, and is based on a predetermined heat flux. We already showed that the two are not the same. For example, when the flux is fixed, the horizontal surface temperature gradient decreases with increasing Rayleigh number. Obviously, when the impressed temperature is specified, $T_{y}$ at the surface is fixed. These observations are related to the fact that when the flux is specified the boundary layer character of the solution is more pronounced, and the forcing is strongest next to the coldest point on the surface. We feel that this difference is important, and even if Roberts results can be applied to the case where the surface temperature is specified, they will not prove sufficient when the flux is given. 


\section{Variable heat flux}

A periodic steady state is reached by driving the circulation with a time dependent forcing representing the four seasons. Realizations of the velocity and temperature fields are produced at the end of the winter and summer seasons for the two Rayleigh numbers, with and without mixing. These are summarized in figures (5.4)-(5.7).

The stream function contours in (5.4-7)a,d clearly show that $\psi$ is stronger in the summer than in the winter, and that at least for the larger Rayleigh number, even though the circulation is stronger, the eye of the gyre was pushed downward in the summer time [figures (5.6-7)a,d]. As expected, the overturning region in the winter is much broader than in the summer and is larger for the mixing case compared to the standard one. When $R_{a}=2 \cdot 10^{4}$ the unstable region reaches the bottom in the summer when no mixing is present, and is confined to the top right corner with mixing. The last is an indication that the strong vertical mixing was successful in eliminating the otherwise unstable stratification. A similar phenomenon can also be observed for $R_{a}=2 \cdot 10^{5}$. There, the unstable region in the bottom right corner, results from advection of the unstable density field. This region erodes when turbulent mixing is assumed.

Comparing the figures, it is also apparent that the unstable region is larger when the Rayleigh number is smaller; a direct result of the effects of the circulation. If there where no flow field, ( $R_{a}=0$, diffusive case of the second chapter), and there is no turbulent mixing, the analytic solution (2.2) shows that the line separating the two regions is vertical. When we start increasing the circulation, the line moves towards the colder wall [figure $(5.1-2) \mathrm{a}, \kappa=1$ ]. The stronger the circulation, the more surface trapped the unstable region is, and the narrower the strip it occupies next to the northern boundary.

In addition to looking at snapshots of the various fields, it is also very instructive to analyze the time development of $\psi_{\max }$ in a yearly cycle. This is done in figure (5.8) 

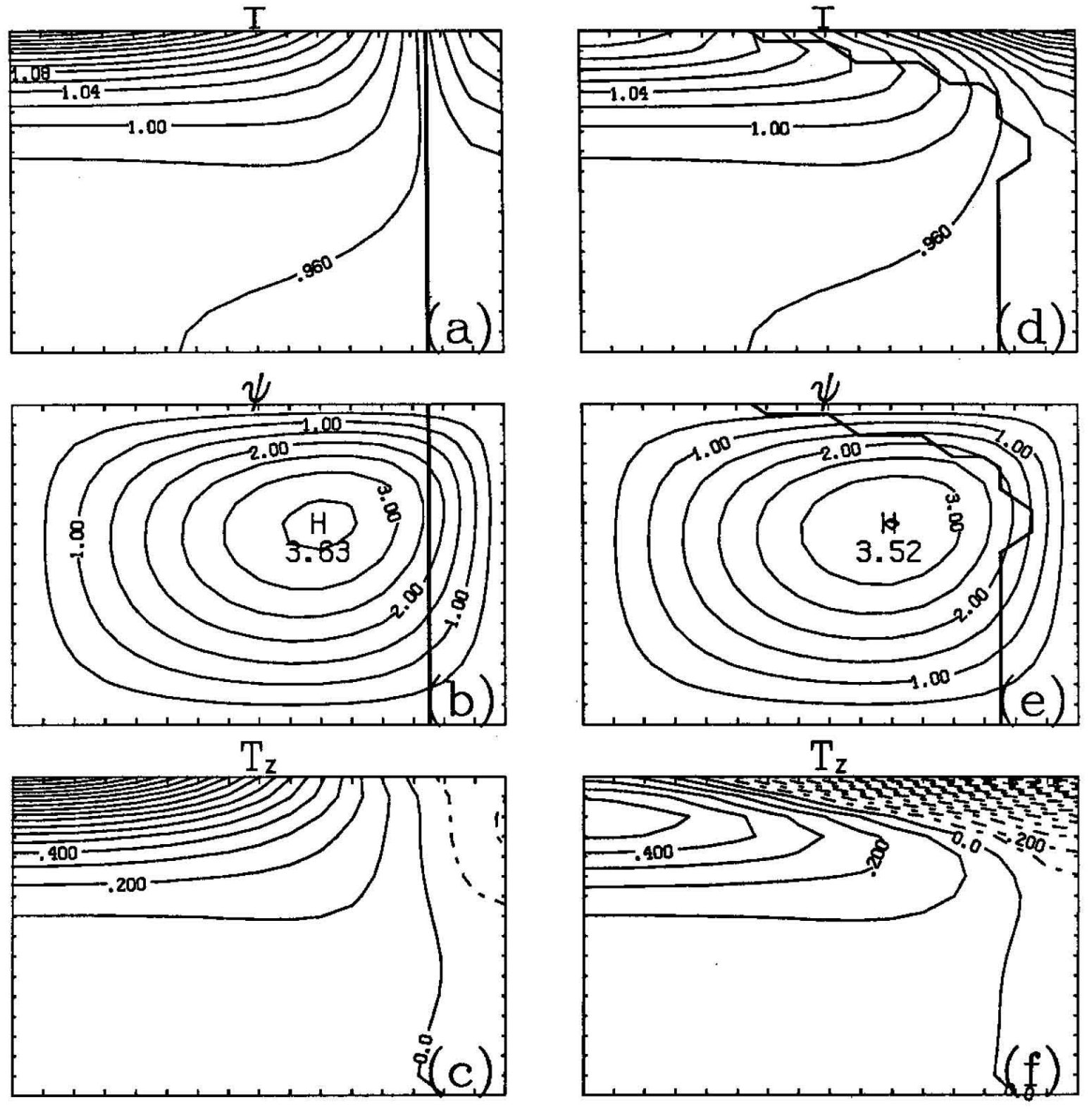

Figure (5.4). The periodic steady state when seasonal cycle is imposed. The first column is for the situation at the end of summer, while the second is evaluated at the end of winter. This is the standard case with $R_{a}=2 \cdot 10^{4}$. (a), (d) $\psi$. (b), (e) T. (c), (f) $T_{z}$. 

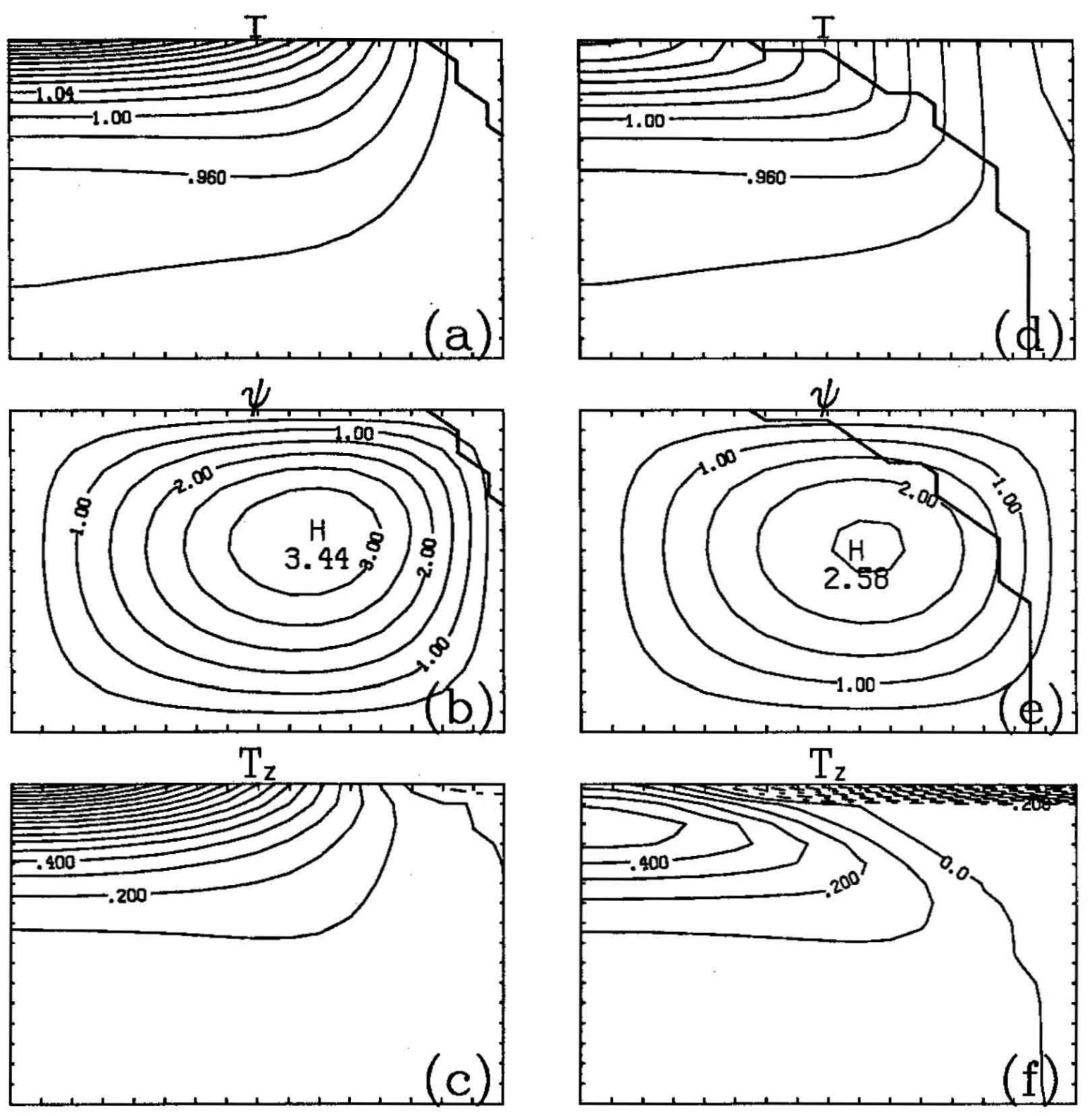

Figure (5.5). The same as (5.4) but with mixing. 

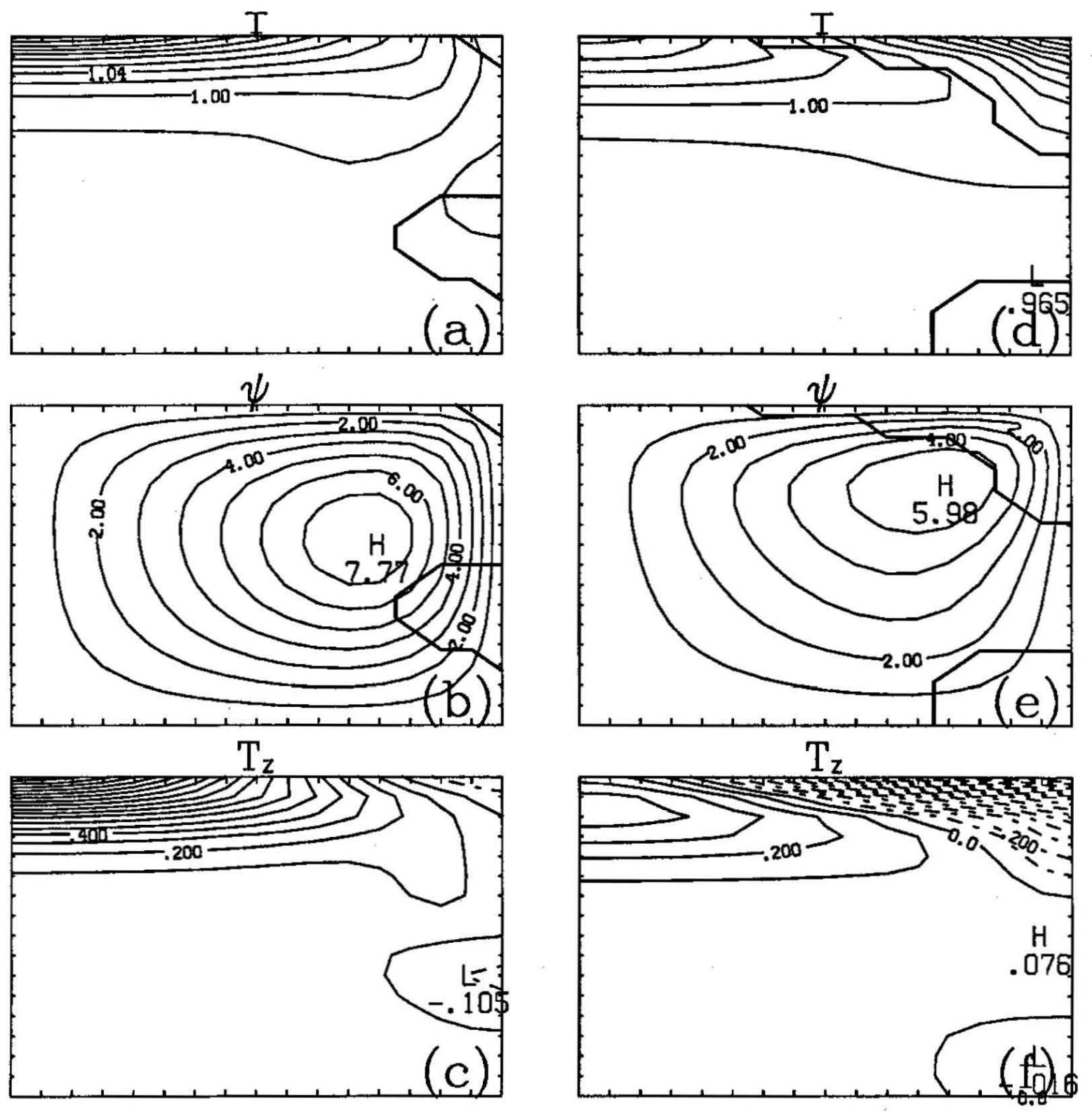

Figure (5.6). The same as (5.4) but with $R_{a}=2 \cdot 10^{5}$. 

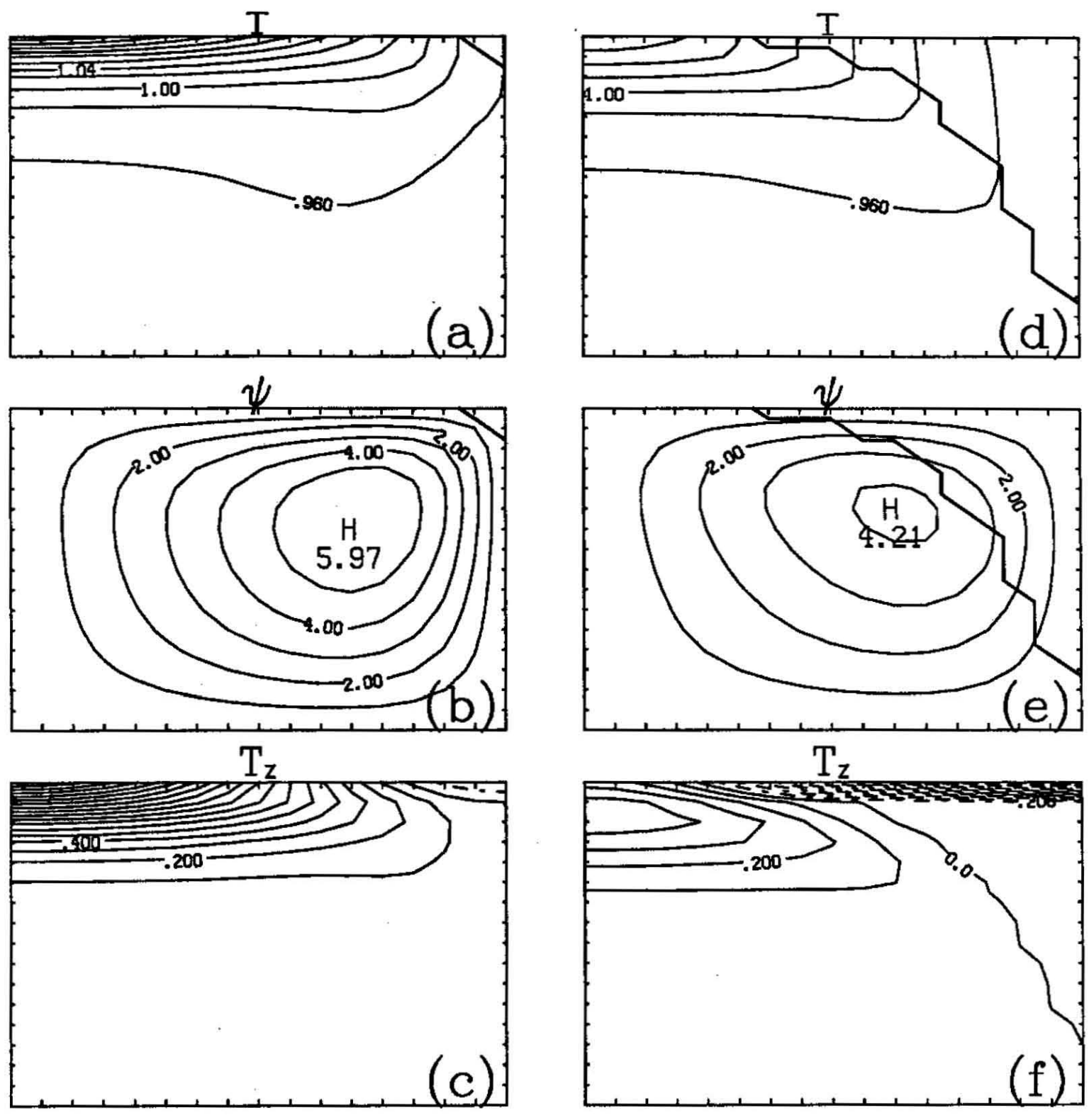

Figure (5.7). The same as (5.5) but with $R_{a}=2 \cdot 10^{5}$. 
for the two Rayleigh numbers, with and without mixing. It is apparent from these presentations that there are two time scales in the problem; a scale for the forcing, and a scale for the response. The phase difference between the forcing and the response is also evident. For example, in the standard case with $R_{a}=2 \cdot 10^{5}$, [figure (5.8) line $\mathrm{c}] \psi_{\max }$ decreases throughout most of the summer and increases through most of the winter. When the Rayleigh number is smaller by an order of magnitude, the situation changes. In figure (5.8) line a $\psi_{\max }$ increases through most of the summer, and decreases in the last quarter of the heating period. $\psi_{\max }$ starts increasing only towards the end of the winter season.

In order to understand the relation between the stream function, the forcing, and the Rayleigh number, it is beneficial to investigate the results of steady summer or winter forcing. These correspond to permanent summer or winter conditions. The heat content of the system will no longer be constant over any periodic cycle, but both the temperature structure (not the absolute values, but $T_{y}$ and $T_{z}$ ) and the stream function reach a steady state.

These experiments are motivated by simple questions arising from the previous figures. For example, figure (5.8) suggest that $\psi_{\max }$ attains its maximum value somewhere in the summer time, and minimum value in the winter time. But we just might find out that the circulation is much more intense for persisting winter condition than for permanent summer ones. This will hint that there is a possible lag of about half a year between the forcing and the response. Other questions, relating the Rayleigh number and the difference in the time scale it takes to reach the quasi winter steady state as opposed to the summer one, will also be addressed, helping in our understanding why in figure (5.8) lines a and $c$ are symmetric while lines $b$ and $d$ are not.

The results when permanent heating or cooling is applied are presented in figures (5.9-11). They show several distinct features, the most prominent of which is that indeed the circulation is much stronger in the winter than in the summer. In the 


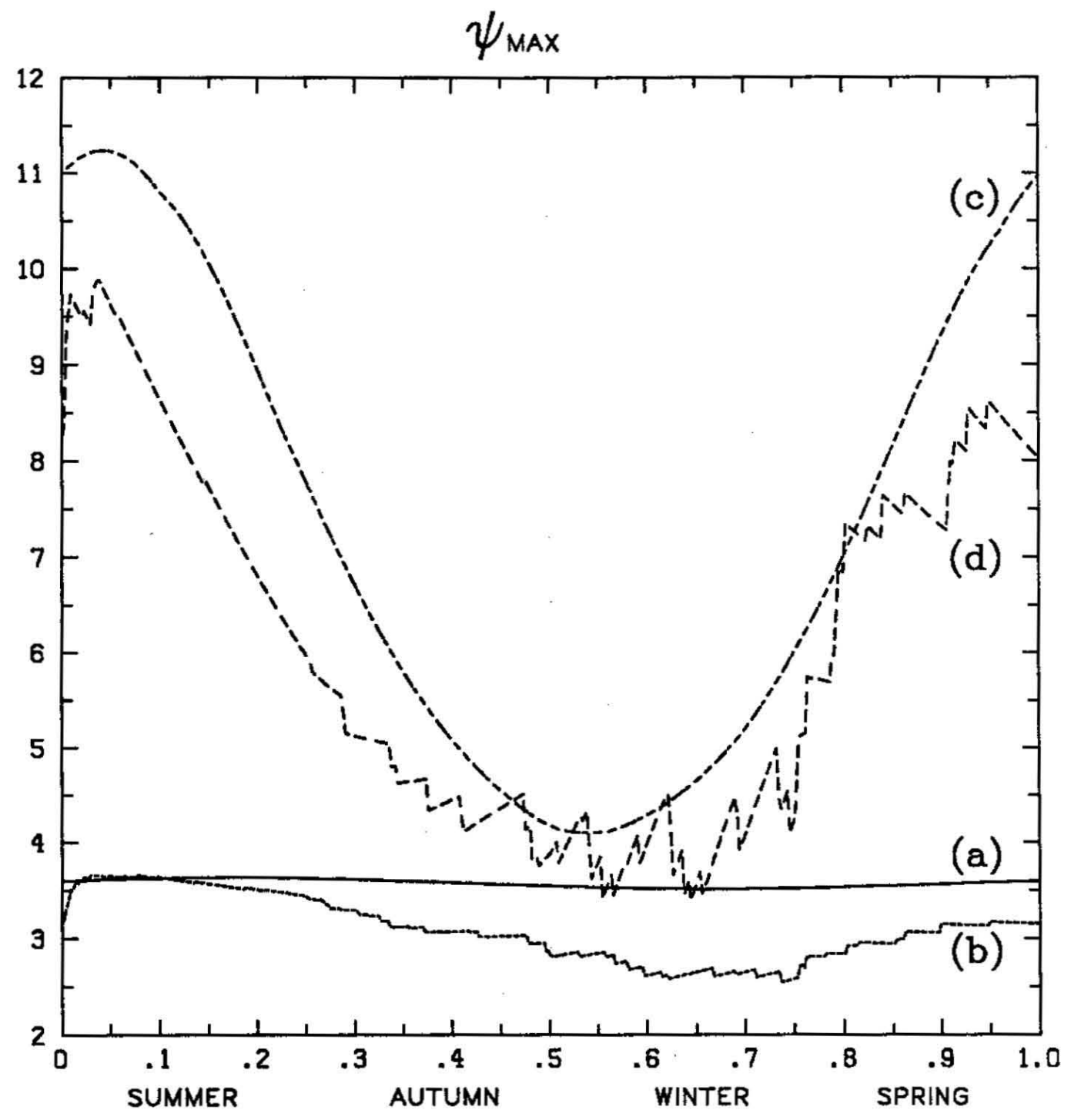

Figure (5.8). Evolution of $\psi_{\max }$ over a year. Line (a) corresponds to the standard case, (b) to the turbulent mixing case, both with $R_{a}=2 \cdot 10^{4}$. Lines (c) and (d) are the same, but with $R_{a}=2 \cdot 10^{5}$. 
winter, a large portion of the basin is being cooled hence its effective Rayleigh number is smaller; thus, $\psi_{\max }$ decreases going from the standard case to mixing one. The location of the maximum value of the stream function moves down and to the south as the effects of mixing becomes dominant because obviously $\psi$ cannot attain its maximum value at the well-mixed region where the gradients are weak. For continuous summer conditions, the results are unaffected by the degree of mixing because of the negligible size of the unstable region. This is why only one realization from summer is presented in figure (5.11). The circulation is confined to the upper domain, and is shallower for larger Rayleigh numbers. The extent of the overturning region is broader in the winter and is a touch larger for the small Rayleigh number, where the circulation is weaker. As was mentioned before, the absolute magnitude of the temperature contours is not important because of the constant heat source (or sink) in the system.

The next set of figures (5.12) represent the evolution of $\psi_{\max }$ as permanent summer or winter conditions are imposed. The two lines in each box correspond to the two different mixing hypotheses. The idea behind these illustrations is to start from an initial condition of the quasi steady state of a particular Rayleigh number, mixing parameterization, and summer or winter forcing and to impose the opposite heating function. By tracing the evolution of $\psi_{\max }$ with time, an appropriate time scale for the response to the onset of cooling and heating can be determined. It is important to note the changes that $\psi_{\max }$ undergoes on its way to the its final value, and the dependence of the process on the Rayleigh number. For example, when going from winter to summer in figures (5.12)b, $\mathrm{d}$ the transition is monotonic for the standard case, but has a large peak followed by a fast decay in the mixing case.

In summary, four sets of figures are available to investigate the response to a forced seasonal cycle. They include contours of the fields at the end of the summer and winter seasons [figures (5.4-7)], and after permanent winter or summer conditions [figures (5.910)]. The time evolution of $\psi_{\max }$ through a cycle [figure (5.8)], and from permanent 

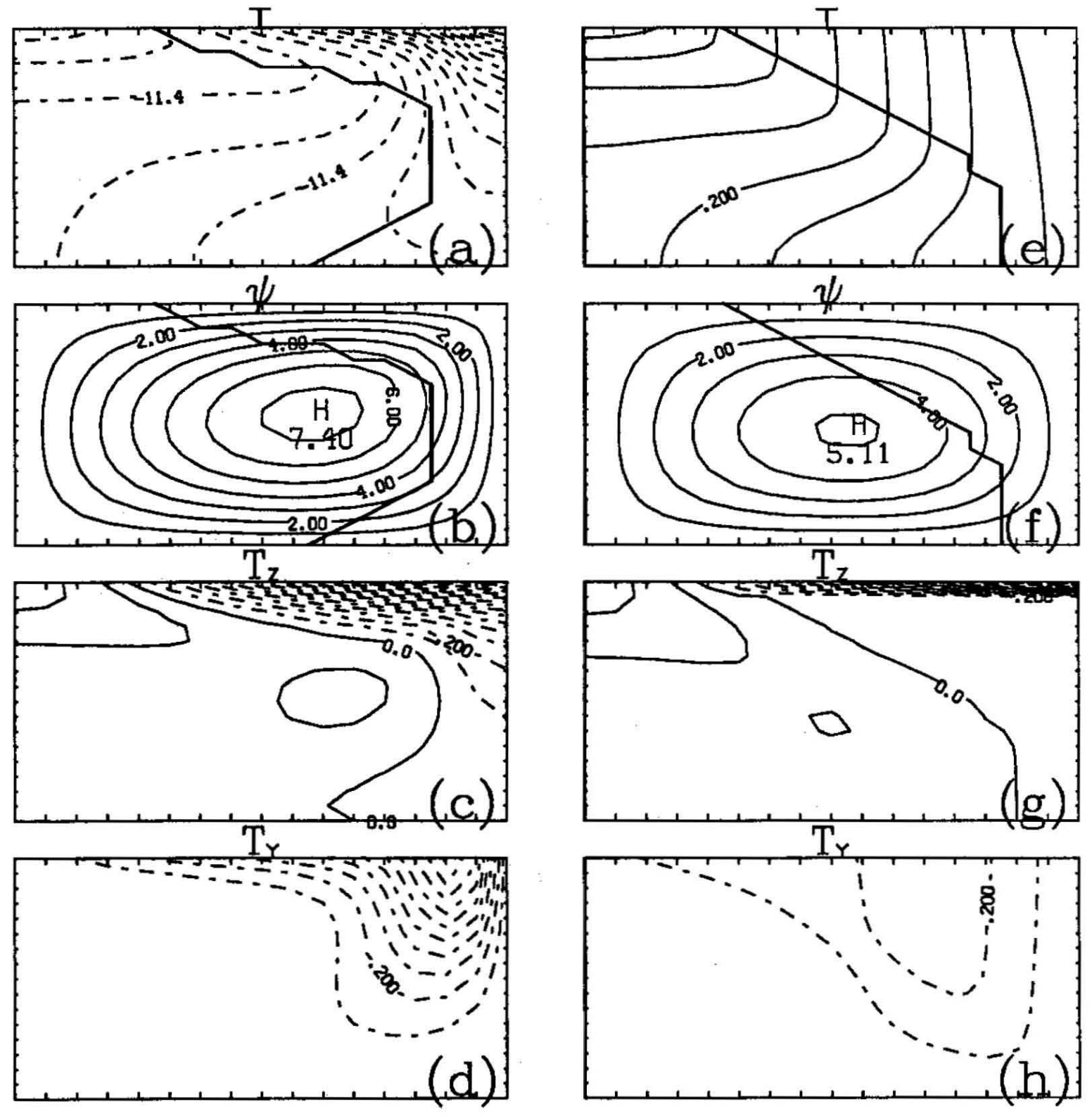

Figure (5.9). Quasi steady state for permanent winter condition when $R_{a}=2 \cdot 10^{4}$. The first column represent the fields in the standard case while the second corresponds to the mixing case. (a), (e) $\psi$. (b), (f) $T$. (c), (g) $T_{z}$. (d), (h) $T_{y}$. 

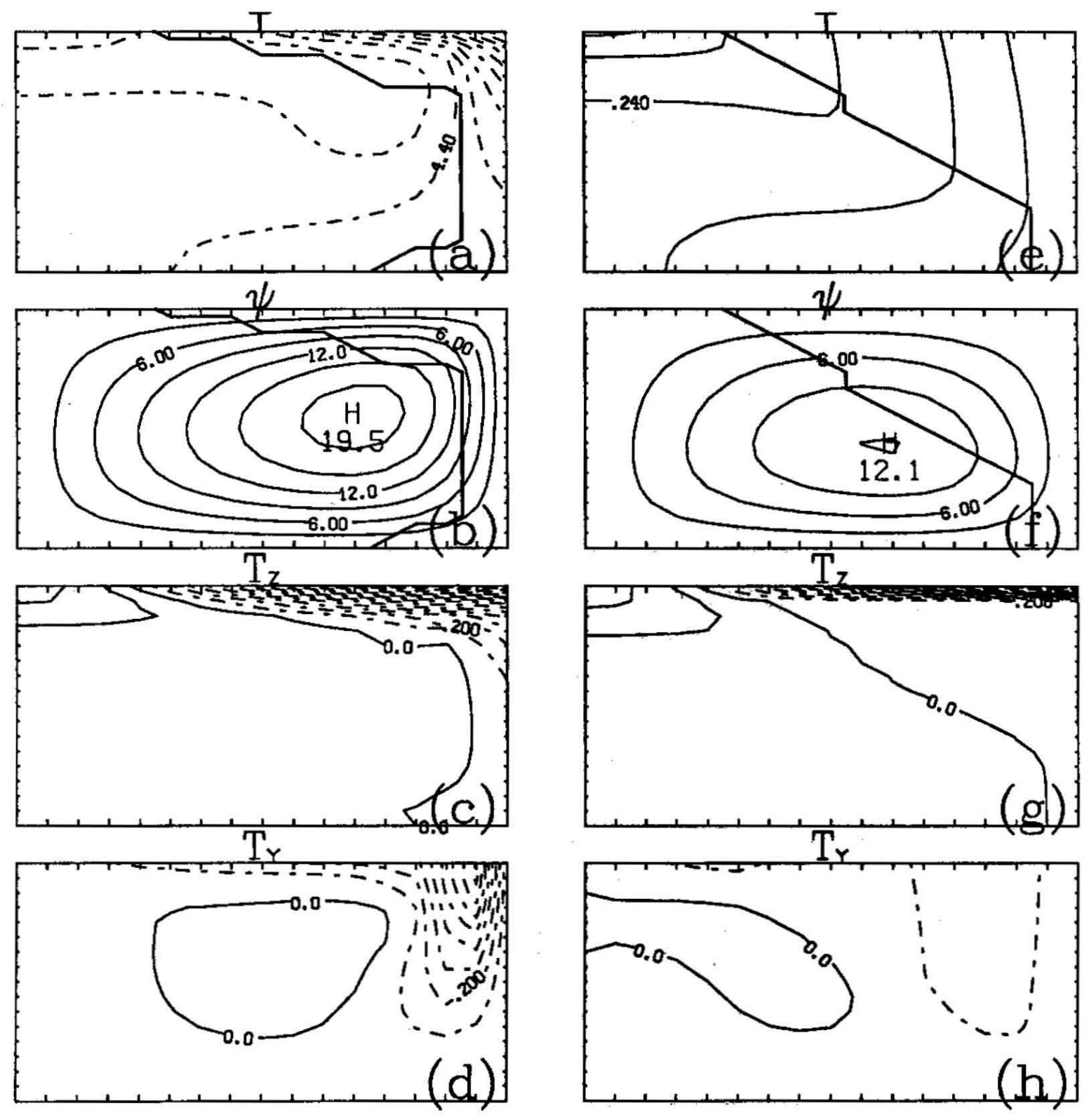

Figure (5.10). The same as (5.9) but for $R_{a}=2 \cdot 10^{5}$. 

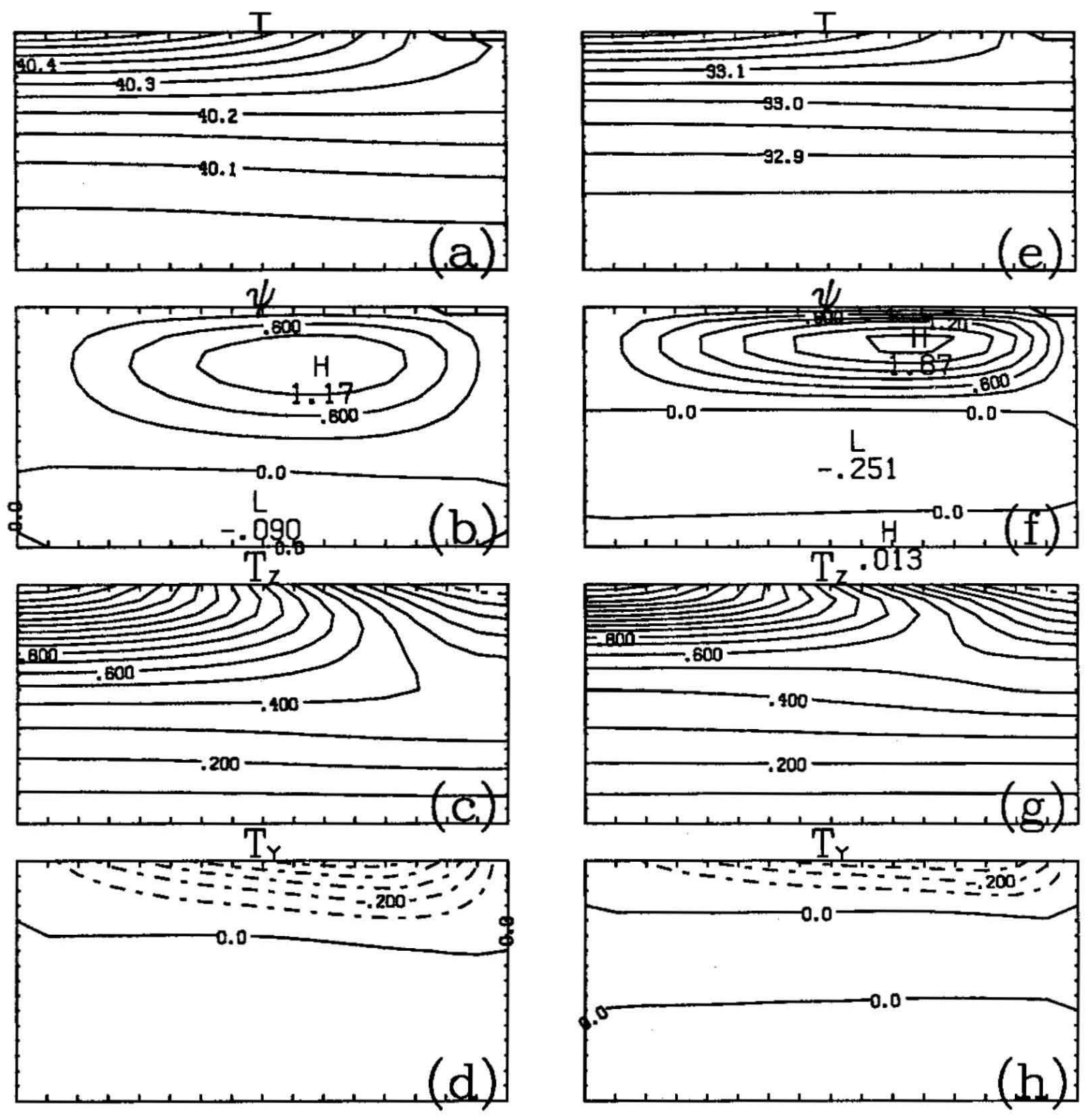

Figure (5.11). Quasi steady state for permanent summer condition. In the first column $R_{a}=2 \cdot 10^{4}$ while in the second $R_{a}=2 \cdot 10^{5}$. The summer state is not affected by the mixing hypothesis. (a), (e) $\psi$. (b), (f) $T$. (c), (g) $T_{z}$. (d), (h) $T_{y}$. 

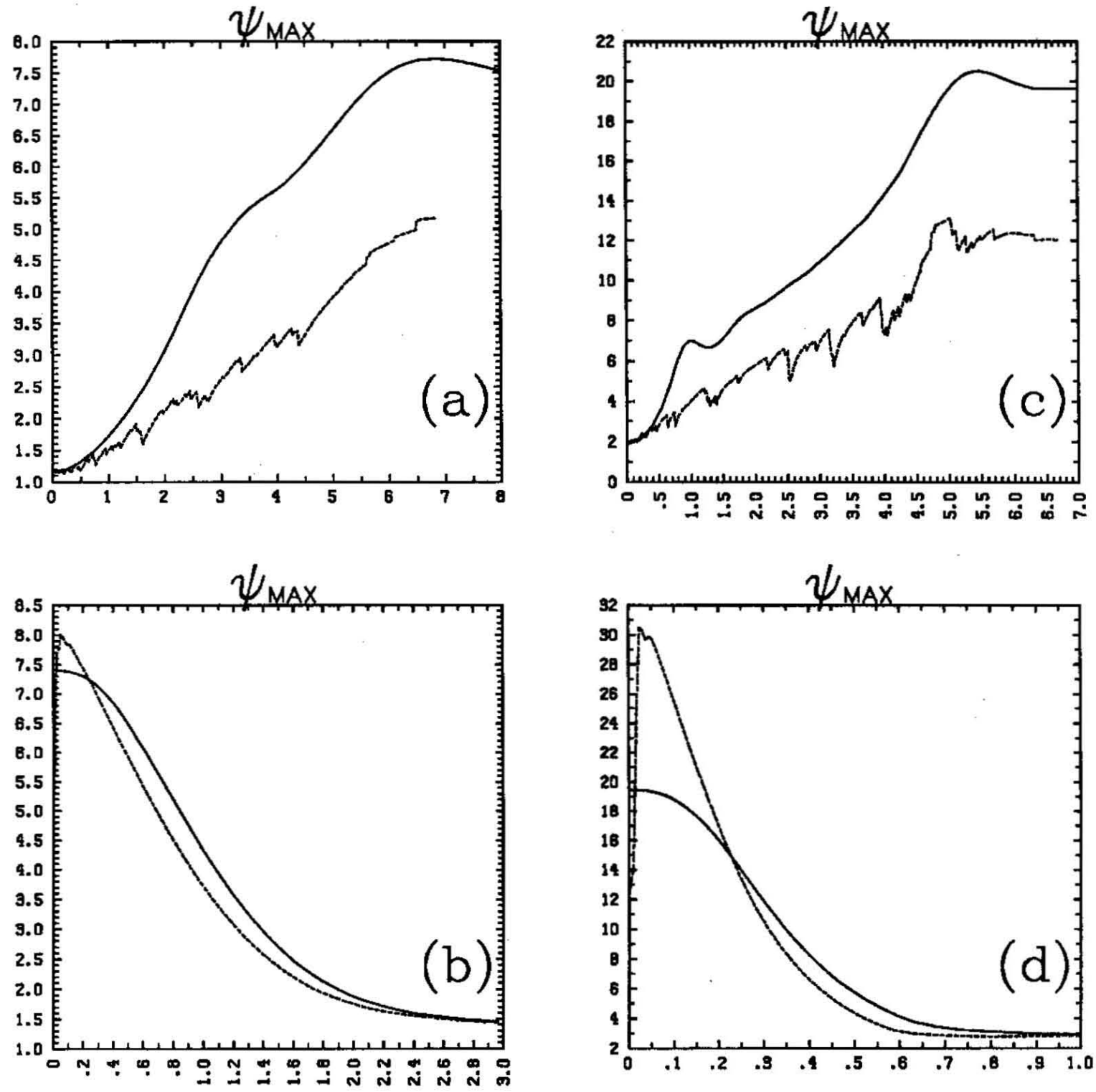

Figure (5.12). Evolution of $\psi_{\max }$ while permanent summer or winter conditions are imposed. The solid line corresponds to the standard case, while the dashed to density mixing. The units on the abscissa are real time in years. (a) $R_{a}=2 \cdot 10^{4}$, summer to winter. (b) $R_{a}=2 \cdot 10^{4}$, winter to summer. (c) $R_{a}=2 \cdot 10^{5}$, summer to winter. (d) $R_{a}=2 \cdot 10^{5}$, winter to summer. 
summer to winter and back [figures (5.12)] are also included. These figures are drawn for two different Rayleigh numbers and the two mixing cases discussed before.

The last set of figures (5.13)-(5.14) are the mean of the four seasons, the first for $R_{a}=2 \cdot 10^{4}$ with and without mixing, and the second for the higher Rayleigh number. As was done in chapter 2 figure (2.7), these presentations correspond to a sum over four seasonal realizations, whereas (5.1-2) are the accompanying situations when the fluid is forced by the mean forcing which has a simple cosine structure.

\section{Discussion}

The response of the ocean to a seasonally varying forcing is understood better if we know what happens in a spin up problem when the forcing is fixed in time. For that case, figures (5.9-12) illustrate the results when permanent winter or summer conditions are imposed. In addition to the contouring the fields in the steady state, figure (5.12) details the time scales associated with the spin up problem by following the values of $\psi_{\max }$. Several features are illustrated through the figures.

1) The results of summer conditions are approximately the same irrespective of mixing. This is attributed to the very small region in which the stratification is unstable, and is the reason only one permanent summer condition was presented for each Rayleigh number [figure (5.11)].

2) There is the usual tendency in the winter to have larger $\psi_{\max }$ with larger Rayleigh numbers, and smaller when mixing is incorporated. This is apparent comparing (5.9)a to (5.10)a and (5.9)e to (5.10)e.

3) $\psi_{\max }^{\text {winter }} \gg \psi_{\max }^{\text {summer }}$ as is evident from (5.9-10)a in comparison to (5.11)a and (5.11)e.

4) The decay of $\psi_{\max }^{\text {winter }}$ to $\psi_{\max }^{\text {summer }}$ is fast, is accomplished in order of a year [figure (5.12)b,d], and when mixing is available, peaks strongly before its sharp decline. 

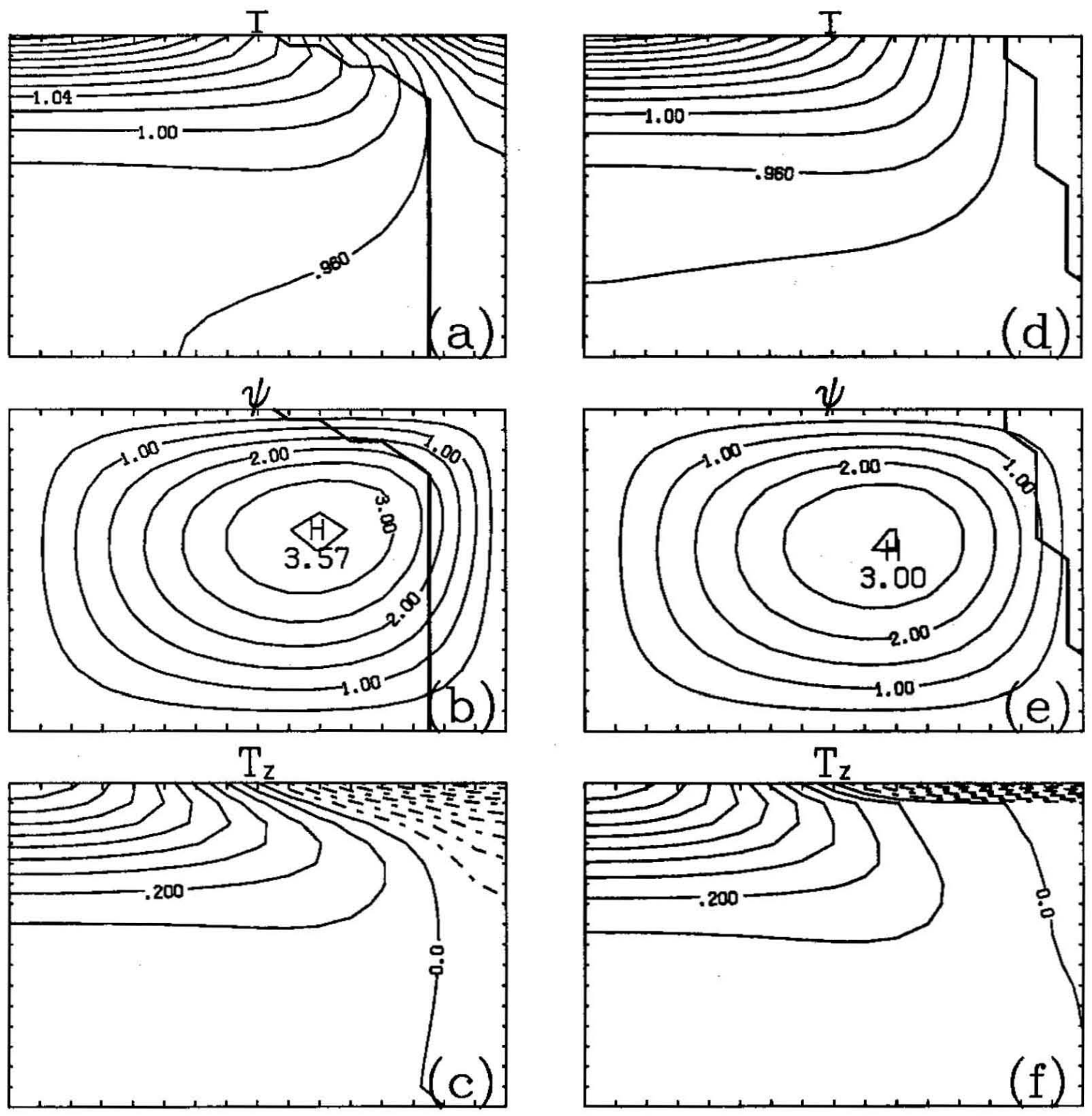

Figure (5.13). The mean fields from the four seasons with $R_{a}=2 \cdot 10^{4}$. Standard case shown in the first column, density mixing in the second. (a), (d) $\psi$. (b), (e) T. (c), (f) $T_{z}$. 

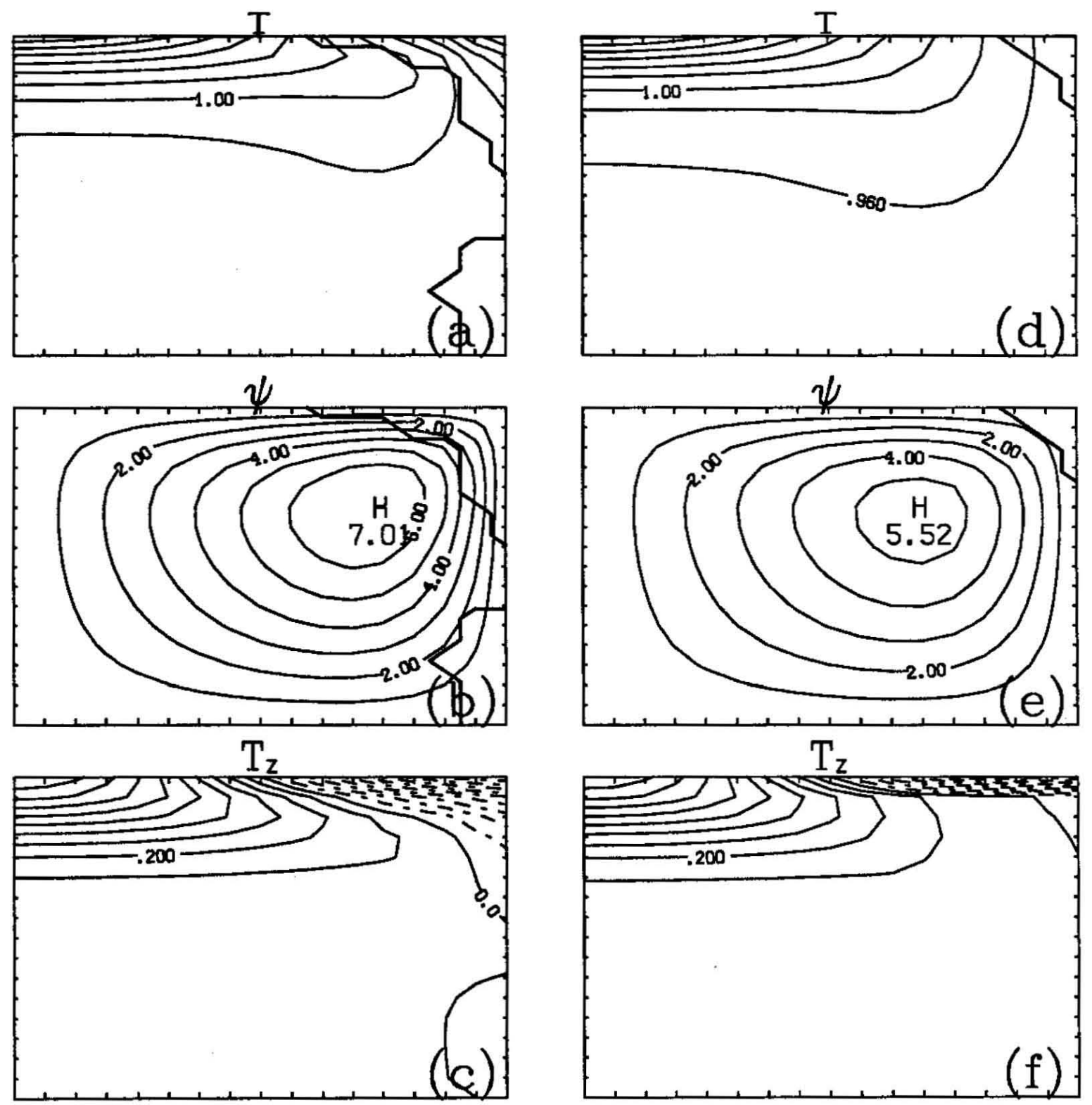

Figure (5.14). The same as (5.13) but for $R_{a}=2 \cdot 10^{5}$. 
5) The rise from $\psi_{\max }^{\text {summer }}$ to $\psi_{\max }^{\text {winter }}$ is slower, takes around six years [figure (5.12)a,c] and is relatively steady.

The explanation of the items above is based on several simple arguments. From previous experience-and opposite to what is suggested in the third point above-since in the winter the effective Rayleigh number is smaller over a large portion of the domain, the circulation should be weaker. This result was examined before for fixed forcing. Compare for instance figures (5.1)a,d,g to (5.2)a,d,g and note that $\psi_{\max }$ decreases going from the standard to the mixing case. A similar tendency is mentioned in the second item above. But the same behavior is no longer true when comparing different forcing, one with intense cooling, and one with heating. When summer conditions prevail, the unstable region is almost nonexistent, and mixing or not, the effective Rayleigh number is fixed apart from the top right corner. For our choice of geometry and forcing, this area is too small to affect the quasi steady state solution. In the winter, the overturning region is much broader and extends - at least next to the surface-to the southern portion of the domain. As always, within the winter season, $\psi_{\max }$ decreases as the effects of mixing reduce the effective Rayleigh number.

The reasoning for the observations leading to the third point above lies in the size of $T_{y}$ as seen in figures $(5.9) \mathrm{d}, \mathrm{h},(5.10) \mathrm{d}, \mathrm{h}$, and (5.11) $\mathrm{d}, \mathrm{h}$. In the winter time this term is much larger than in the summer, in particular next to the surface and along the northern jet. Because in addition to the surface boundary condition this is what actually forces the velocity field at all interior points, $\psi$ increases considerably in the cooling period. The physics behind this description is related to the different density layering observed in (5.9) b,f, (5.10) b,f, and (5.11) b,f. In the summer time the temperature contours are more flat so $T_{y}$ is small, whereas in the winter the temperature next to the surface is more uniform in the vertical so $T_{y}$ increases.

When relaxing from winter to summer in the standard case, horizontal diffusion acts upon the large $T_{y}$ gradients - which now have no support from the forcing-thus, 
$\psi_{\max }$ decreases monotonically to its summer value described in figures (5.12)b,d line a. If turbulent mixing is available, the situation is very different and the circulation is intensified over a very short time to reach a maximum from which it decays fast to its summer quasi steady state.

When starting with abrupt summer conditions after a winter quasi steady state, there is a large increase in $T_{y}$ values next to the surface in the northern region. The particular location of this sudden change in forcing is important because this is the location where small changes in forcing the elliptic problem have the most pronounced effect on the circulation. Compare for instance the $T_{y}$ contours in figure (5.9)h to those of (5.11)d. The figures show that the $T_{y}$ values in the summer are larger than those in the winter when mixing is present. On the other hand, where there no mixing as in (5.9)d, the $T_{y}$ values in the winter are large as well. When mixing is present, the strong $T_{y}$ gradients causes a sudden increase in the flow field which gradually decays to the permanent summer condition. In particular, when the Rayleigh number is large, the circulation is stronger and decays faster. The last process is aided by horizontal diffusion erasing the signature of the strong $T_{y}$. For example, figure (5.12)b line b with $R_{a}=2 \cdot 10^{4}$ decays on a time scale that is four times longer than line $\mathrm{b}$ in figure (5.12)d where $R_{a}=2 \cdot 10^{5}$.

The transition from summer to winter [figure (5.12)a,c] is slower and takes around six to seven years, again depending on the Rayleigh number. In the adjustment process, $\psi_{\max }$ overshoots its final winter value, and then decays back to it. When no mixing is present the procedure is smooth, but otherwise is very wiggly. This bumpiness in the figures is due to time stepping with low vertical resolution. Deepening the interface by one grid point at a given latitude requires a certain number of time steps depending on the amount of heat that need be removed from the water column, in order for its density to be as heavy as the fluid a grid point below it. While this cooling occurs, the interface does not change and the stream function has a short period in which to adjust 
to the density field. Then, in one step the interface all of a sudden moves, changing the effective Rayleigh number. $\psi_{\max }$ will change abruptly and would go through a short adjustment stage as before, until the interface moves no more. The picture described above indicates that if a cycle on earth were to take around 10 years or more, then the circulation would reach its maximum in the colder period, and minimum in the warmer ones. In that case, the response to the forcing would look like a combination of the corresponding lines in figures (5.12) $\mathrm{a}-\mathrm{b}$ or $(5.12) \mathrm{c}-\mathrm{d}$, and would not be symmetric even in the standard case, in contrast to the symmetry of line a in figure (5.8).

Knowledge of this permanent cooling and permanent heating condition is valuable in evaluating the results from a cyclic forcing. It shows immediately that there is a phase difference of several months between the forcing and the response, because for permanent winter the circulation is at its maximum, whereas in the seasonal cycle it reaches a minimum. This phase can be described by the difference in time between the minimum and maximum of $\psi_{\max }$ in figure (5.8), and the relationship between these values and the season. As mentioned before, it is also apparent that the symmetry of the response in figure (5.8) lines a and $c$ is not something typical of the system, but is rather an artifact of the very short time scale in which the differences between cooling and heating do not have enough time to surface.

When there is no turbulent mixing as in figures (5.1) a-c, and (5.2)a-c, the cycle looks symmetric (as the forcing) and it takes half a year to move between the two extremes of $\psi_{\max }$. But the phase of the response depends on the Rayleigh number. The larger it is, the less into the summer season $\psi_{\max }$ decreases. When $R_{a}=2 \cdot 10^{4}$ [figure (5.8) line a] $\psi_{\max }$ peaks at about $2 / 3$ of the summer and then falls off. For $R_{a}=2 \cdot 10^{5}$ [figure (5.8) line c], the peak is less than a fifth into the season. Because of the symmetry of the forcing, the same (replacing maximum by minimum) holds for the winter period. There, the intensity of the circulation is increased more towards the beginning of the season as the Rayleigh number increases. 
When mixing is introduced, the problem becomes inherently nonlinear (even if one still wants to approximate the balance in the heat equation by vertical advection against vertical diffusion) and in addition, the effective Rayleigh number varies. Although the forcing is still symmetric in the sense that there is warming for half the period and cooling for the other half, the response is not. In figure (5.8) line $\mathrm{b} \psi_{\max }$ decreases over 0.6 of a year for $R_{a}=2 \cdot 10^{5}$, and 0.7 of a year in the same figure line b when $R_{a}=2 \cdot 10^{4}$. For both Rayleigh numbers, the maximum of $\psi_{\max }$ is reached at the beginning of the summer. The difference is in the shorter time span where $\psi_{\max }$ decreases even though there is cooling. For the larger Rayleigh number in figure (5.8) line d, the minimum of $\psi_{\max }$ is reached 0.5 of a year into the cooling period, (end of winter, when actually heating starts) but when $R_{a}=2 \cdot 10^{4}$ as in figure (5.8) line b, $\psi_{\max }$ starts gaining after a shorter period of 0.4 of a year.

While following the response to a seasonal cycle, it is easier to look at the forcing as two events, summer and winter, separated by two intermediate seasons autumn and spring. At those two seasons the net heating is much smaller than in the main events. The moderate periods allow the system to equilibrate slightly to previous changes before imposing a new severe forcing, thereby smoothing the passage between seasons.

In a schematic cycle starting from the end of summer, $\psi_{\max }$ decreases in value into autumn (where there is slight cooling). The winter is successful in pointing $\psi_{\max }$ again in the right direction and it increases from the end of winter through spring and the beginning of summer. The strong warming in the summer limits $\psi_{\max }$ and it starts decreasing to complete a cycle. The amount of time spent on the downward motion while $\psi_{\max }$ decreases depends on the type of mixing assumed. The vicinity of the bending points where $\partial_{t} \psi_{\max }$ changes sign to the beginning of summer and winter is related to the Rayleigh number.

The difference between mean realizations and mean forcing is seen comparing (5.13)a-c to (5.1)a-c, (5.13) d-f to (5.1)d-f, (5.14)a-c to (5.2)a-c, and (5.14)d-f to 
(5.2)d-f. The changes are not as large as observed in the second chapter because of two factors. The most important one is the efficiency of advection in transporting heat. This process was not included in the chapter 2 , and plays an important role in balancing the system. Additionally, the forcing here is much more well behaved than Oort and Vonder Haar data used before, and is a simple shifted cosine.

For the large Rayleigh number, there is a noticeable weakening of the flow field when averaging the realizations with and without mixing. The thermal structure is nearly unaffected and the temperature for the mean realizations is a bit colder everywhere compared to the mean forcing. Because of that, $\Delta T$ is comparable in both averaging methods. It is 0.25 for the standard case, and 0.18 for the mixing one. $\mathrm{Al}-$ though $\Delta T$ is not changed, the local air sea flux will change because the sea surface temperature is not the same.

When averaging realizations with mixing the overturning region is smaller than the average forcing picture, and is determined by its extent in the warmest period. As was explained in the second chapter this is a result of the summer time stable stratification that can easily overcome any marginally stable regions developed closer to the south in the winter time. 


\section{Conclusions}

The parameterization of turbulent mixing introduced in this work has the desired effect of reducing the effective Rayleigh number, weakening the flow field, and moderating the boundary layer character of the solution. Using previous estimates of $\left(R_{a} \lambda^{2}\right)=$ $2.5 \cdot 10^{11}$, our scaling arguments for a $5 \mathrm{~km}$ ocean depth leads to a thermocline depth of less than $50 \mathrm{~m}$, which is a bit on the shallow side. Because the Rayleigh number in the ocean can probably be this large, the mixing process seems a reasonable way to smooth some of the sharp gradients found otherwise. The addition of meridional boundaries and rotation would presumably help in gaining a more realistic picture of the thermohaline circulation.

Assuming that the total heat content in the ocean is fixed, the mixing parameterization leads to an interior which is somewhat colder than without it resulting in warmer surface temperature in equatorial regions. The unstable stratification in the northern region is eliminated, and the density there is nearly vertically homogeneous. In addition, the mixing process forces the center of the gyre to migrate towards the south and the bottom relative to an ocean without mixing, while the stream lines are more bent.

The response of the ocean to a seasonal cycle follows the regular pattern where the circulation gets stronger as the Rayleigh number increases, and weakens where mixing is assumed.

When the flow is being forced by permanent cooling it is found that the forcing term $T_{y}$ is large and the circulation is strong. This result is intuitively correct remembering that in the winter, more deep water is formed thereby the intensity of the vertical circulation has to increase. The transition from weak circulation (summer) to strong (winter) is much slower than the reverse. The last (relaxation process) is being helped by a positive feedback mechanism to reduce the local gradients everywhere, where in the first (building the circulation) the forcing has to work to generate the conditions needed 
to support the large gradients. Because the seasonal cycle shows a near minimum in the values of $\psi_{\max }$ during the winter time, and near maximum in the summer time, we conclude that the response of the ocean lags the forcing by several months. This is to say that the effects of water mass production in the winter will be observed in the large scale circulation only at the summer time. Assuming the diffusivity and aspect ratio are fixed, the parameters governing this behavior-including the steepness and sign of the slope describing $\psi_{\max }$ during heating and cooling-are the Rayleigh number and the turbulent mixing.

As $R_{a}$ increases, so does the velocity field; this acts to speed up the process and shortens the time required for temperature field to relax. The fact that the seasonal cycle is not as symmetric as the driving force is another manifestation of the idea that advection and diffusion have different efficiencies. The advection carries the density field around fast, while the diffusion is more effective in local homogenization.

Although we were not able to come up with a general mathematical expression for the lag between the forcing and the response, the patterns indicate clearly that an appropriate time scale for a perturbation to decay in the ocean is larger than a season. Otherwise, $\psi_{\max }$ would start increasing while cooling was imposed, and start decreasing in the autumn.

Changes between different averaging procedures are apparent for large Rayleigh number where the surface temperature is colder and the circulation is weaker when mean seasonal picture is compared to mean forcing one. The changes are larger when turbulent mixing is used. 


\section{Concluding remarks}

The climate of our planet has always proved fascinating to its inhabitants, and in that respect, the physical oceanographer is no different. One of the possible angles of approach adopted in oceanography in order to explain the climate is by attempting to understand the thermohaline circulation. Towards that goal, this work investigates several aspects of the axially symmetric circulation. In a time where it is possible to run high resolution primitive equation general circulation models on large computers, this simplification of the fluid's behavior seems to be less popular than twenty years ago. But because the dynamics of the numerical experiments is complicated, their results are hard to analyze and are also sensitive to a large number of parameters. This is the reason why an axially symmetric configuration was chosen here, even though it is obviously less suitable in describing the intricate details of the flow pattern.

Two aspects of our analysis are new for the configuration of the meridional circulation. Those are the inclusion of turbulent mixing parameterization, which is now more common in circulation experiments, and a strong emphasis on variability. In addition, we replaced the boundary condition along the non-insulating wall from an impressed temperature to a specified flux. Although this change seems to be rather minor, this is not always the case because in many problems specifying the temperature results in the actual heat flux forcing being formed as part of the problem, and need not be at all realistic, whereas specifying a given flux is a direct statement of the forcing.

The question of variability is important because even small changes in a large water mass like the ocean can have pronounced climatic effects. In general, there are many scales associated with any particular problem, and because it is hard to account 
for all of them, one usually employs scaling arguments to justify neglecting some and retaining others. In this work, annual variability is introduced through the boundary condition, and the response of the fluid is analyzed.

In addition to numerical experiments, an analytic model is also presented. Comparisons between the two are not really possible because the underlying dynamics is different in the two cases. With that in mind, the analytic model is used to enhance our understanding of the consequences of conservation of potential vorticity in an axially symmetric system.

A model was also developed to describe open water water mass formation in "chimneys". Given a surface buoyancy loss and the local initial stratification this model can predict the penetration depth of the chimneys. We tried to relate the results of this model to the MEDOC observations and to the water mass of the Levantine Intermediate Water. The formation processes in the Eastern Mediterranean are not well understood, in particular, because there are few measurements of the events themselves with no evidence of intense upwelling and downwelling as would be expected if the process involves advection. Our model is especially suitable for these region because the chimneys are generated by vigorous mixing only. 


\section{Appendix}

Evaluation of the Arakawa Jacobian in a uniform grid

As suggested by Arakawa (1966), the Jacobian can be written in all interior points as

$$
\begin{array}{r}
J(\psi, T)=\frac{1}{12 d^{2}}\left[\left(\psi_{i, j-1}+\psi_{i+1, j-1}-\psi_{i, j+1}-\psi_{i+1, j+1}\right)\left(T_{i+1, j}+T_{i, j}\right)-\right. \\
\left(\psi_{i-1, j-1}+\psi_{i, j-1}-\psi_{i-1, j+1}-\psi_{i, j+1}\right)\left(T_{i, j}+T_{i-1, j}\right)+ \\
\left(\psi_{i+1, j}+\psi_{i+1, j+1}-\psi_{i-1, j}-\psi_{i-1, j+1}\right)\left(T_{i, j+1}+T_{i, j}\right)- \\
\left(\psi_{i+1, j-1}+\psi_{i+1, j}-\psi_{i-1, j-1}-\psi_{i-1, j}\right)\left(T_{i, j}+T_{i, j-1}\right)+ \\
\left(\psi_{i+1, j}-\psi_{i, j+1}\right)\left(T_{i+1, j+1}+T_{i, j}\right)- \\
\left(\psi_{i, j-1}-\psi_{i-1, j}\right)\left(T_{i, j}+T_{i-1, j-1}\right)+ \\
\left(\psi_{i, j+1}-\psi_{i-1, j}\right)\left(T_{i-1, j+1}+T_{i, j}\right)- \\
\left.\left(\psi_{i+1, j}-\psi_{i, j-1}\right)\left(T_{i, j}+T_{i+1, j-1}\right)\right]
\end{array}
$$

where information from 8 points surrounding the point of interest is used to determine the Jacobian, and the grid configuration stretches from 0 to $n$ in the first coordinate, and from 0 to $m$ in the second. Along the boundaries, not all the information is available (assuming one does not create artificial points outside the domain), and in addition the grid box is smaller. It is half the area of an interior box at all boundary points except the four corners where it is a quarter. It is still possible to utilize the same scheme to get the following expressions for the boundary and corner terms. 


\section{Bottom}

$$
\begin{array}{r}
J(\psi, T)=\frac{1}{12 d^{2}}\left[\left(\psi_{i+1,0}+\psi_{i+1,1}-\psi_{i-1,0}-\psi_{i-1,1}\right)\left(T_{i, 1}+T_{i, 0}\right)+\right. \\
\left(\psi_{i, 0}+\psi_{i+1,0}-\psi_{i, 1}-\psi_{i+1,1}\right)\left(T_{i+1,0}+T_{i, 0}\right)- \\
\left(\psi_{i-1,0}+\psi_{i, 0}-\psi_{i-1,1}-\psi_{i, 1}\right)\left(T_{i, 0}+T_{i-1,0}\right)+ \\
\left(\psi_{i+1,0}-\psi_{i, 1}\right)\left(T_{i+1,1}+T_{i, 0}\right)+ \\
\left.\left(\psi_{i, 1}-\psi_{i-1,0}\right)\left(T_{i-1,1}+T_{i, 0}\right)\right]
\end{array}
$$

Top

$$
\begin{array}{r}
J(\psi, T)=\frac{1}{12 d^{2}}\left[\left(\psi_{i, m-1}+\psi_{i+1, m-1}-\psi_{i, m}-\psi_{i+1, m}\right)\left(T_{i, m}+T_{i+1, m}\right)-\right. \\
\left(\psi_{i-1, m-1}+\psi_{i, m-1}-\psi_{i-1, m}-\psi_{i, m}\right)\left(T_{i, m}+T_{i-1, m}\right)- \\
\left(\psi_{i+1, m-1}+\psi_{i+1, m}-\psi_{i-1, m-1}-\psi_{i-1, m}\right)\left(T_{i, m}+T_{i, m-1}\right)- \\
\left(\psi_{i, m-1}-\psi_{i-1, m}\right)\left(T_{i, m}+T_{i-1, m-1}\right)+ \\
\left.\left(\psi_{i+1, m}-\psi_{i, m-1}\right)\left(T_{i, m}+T_{i+1, m-1}\right)\right]
\end{array}
$$

Left

$$
\begin{array}{r}
J(\psi, T)=\frac{1}{12 d^{2}}\left[\left(\psi_{0, j-1}+\psi_{1, j-1}-\psi_{0, j+1}-\psi_{1, j+1}\right)\left(T_{1, j}+T_{0, j}\right)+\right. \\
\left(\psi_{1, j}+\psi_{1, j+1}-\psi_{0, j}-\psi_{0, j+1}\right)\left(T_{0, j+1}+T_{0, j}\right)- \\
\left(\psi_{1, j-1}+\psi_{1, j}-\psi_{0, j-1}-\psi_{0, j}\right)\left(T_{0, j}+T_{0, j-1}\right)+ \\
\left(\psi_{1, j}-\psi_{0, j+1}\right)\left(T_{1, j+1}+T_{0, j}\right)- \\
\left.\left(\psi_{1, j}-\psi_{0, j-1}\right)\left(T_{0, j}+T_{1, j-1}\right)\right]
\end{array}
$$

Right

$$
\begin{array}{r}
J(\psi, T)=\frac{1}{12 d^{2}}\left[\left(\psi_{n-1, j-1}+\psi_{n-1, j}-\psi_{n, j-1}-\psi_{n, j}\right)\left(T_{n, j}+T_{n, j-1}\right)-\right. \\
\left(\psi_{n-1, j-1}+\psi_{n, j-1}-\psi_{n-1, j+1}-\psi_{n, j+1}\right)\left(T_{n, j}+T_{n-1, j}\right)- \\
\left(\psi_{n-1, j}+\psi_{n-1, j+1}-\psi_{n, j}-\psi_{n, j+1}\right)\left(T_{n, j}+T_{n-1, j}\right)- \\
\left(\psi_{n, j-1}-\psi_{n-1, j}\right)\left(T_{n, j}+T_{n-1, j-1}\right)+ \\
\left.\left(\psi_{n, j+1}-\psi_{n-1, j}\right)\left(T_{n-1, j+1}+T_{n, j}\right)\right]
\end{array}
$$


Bottom left corner

$$
\begin{array}{r}
J(\psi, T)=\frac{1}{12 d^{2}}\left[\left(\psi_{0,0}+\psi_{1,0}-\psi_{0,1}-\psi_{1,1}\right)\left(T_{0,0}+T_{1,0}\right)-\right. \\
\left(2 \psi_{1,0}-2 \psi_{0,1}+\psi_{0,0}-\psi_{1,1}\right)\left(T_{0,0}+T_{0,1}\right)+ \\
\left.\left(\psi_{1,0}-\psi_{0,1}\right)\left(T_{0,0}+T_{1,1}\right)\right]
\end{array}
$$

or

$$
\begin{array}{r}
J(\psi, T)=\frac{1}{12 d^{2}}\left[\left(\psi_{1,0}+\psi_{1,1}-\psi_{0,0}-\psi_{0,1}\right)\left(T_{0,1}+T_{1,0}\right)-\right. \\
\left(2 \psi_{1,0}-2 \psi_{0,1}+\psi_{1,1}-\psi_{0,0}\right)\left(T_{0,0}+T_{1,0}\right)+ \\
\left.\left(\psi_{1,0}-\psi_{0,1}\right)\left(T_{0,0}+T_{1,1}\right)\right]
\end{array}
$$

Bottom right corner

$$
\begin{array}{r}
J(\psi, T)=\frac{1}{12 d^{2}}\left[\left(\psi_{n, 1}+\psi_{n-1,1}-\psi_{n, 0}-\psi_{n-1,0}\right)\left(T_{n-1,0}+T_{n, 0}\right)-\right. \\
\left(2 \psi_{n, 1}-2 \psi_{n-1,0}+\psi_{n-1,1}-\psi_{n, 0}\right)\left(T_{n, 0}+T_{n, 1}\right)+ \\
\left.\left(\psi_{n, 1}-\psi_{n-1,0}\right)\left(T_{n-1,1}+T_{n, 0}\right)\right]
\end{array}
$$

or

$$
\begin{array}{r}
J(\psi, T)=\frac{1}{12 d^{2}}\left[\left(\psi_{n, 0}+\psi_{n, 1}-\psi_{n-1,0}-\psi_{n-1,1}\right)\left(T_{n, 1}+T_{n, 0}\right)-\right. \\
\left(2 \psi_{n, 1}-2 \psi_{n-1,0}+\psi_{n, 0}-\psi_{n-1,1}\right)\left(T_{n, 0}+T_{n-1,0}\right)+ \\
\left.\left(\psi_{n, 1}-\psi_{n-1,0}\right)\left(T_{n-1,1}+T_{n, 0}\right)\right]
\end{array}
$$

Top left corner

$$
\begin{array}{r}
J(\psi, T)=\frac{1}{12 d^{2}}\left[\left(\psi_{0, m-1}+\psi_{1, m-1}-\psi_{0, m}-\psi_{1, m}\right)\left(T_{1, m}+T_{0, m}\right)-\right. \\
\left(2 \psi_{0, m-1}-2 \psi_{1, m}+\psi_{1, m-1}-\psi_{0, m}\right)\left(T_{0, m}+T_{0, m-1}\right)- \\
\left.\left(\psi_{1, m}-\psi_{0, m-1}\right)\left(T_{1, m-1}+T_{0, m}\right)\right]
\end{array}
$$

or

$$
\begin{array}{r}
J(\psi, T)=\frac{1}{12 d^{2}}\left[\left(\psi_{0, m-1}+\psi_{0, m}-\psi_{1, m-1}-\psi_{1, m}\right)\left(T_{0, m}+T_{0, m-1}\right)+\right. \\
\left(2 \psi_{1, m}-2 \psi_{0, m-1}+\psi_{1, m-1}-\psi_{0, m}\right)\left(T_{1, m}+T_{0, m}\right)- \\
\left.\left(\psi_{1, m}-\psi_{0, m-1}\right)\left(T_{1, m-1}+T_{0, m}\right)\right]
\end{array}
$$


Top right corner

$$
\begin{array}{r}
J(\psi, T)=\frac{1}{12 d^{2}}\left[\left(\psi_{n-1, m}+\psi_{n, m}-\psi_{n-1, m-1}-\psi_{n, m-1}\right)\left(T_{n-1, m}+T_{n, m}\right)+\right. \\
\left(2 \psi_{n, m-1}-2 \psi_{n-1, m}+\psi_{n-1, m-1}-\psi_{n, m}\right)\left(T_{n, m-1}+T_{n, m}\right)- \\
\left.\left(\psi_{n, m-1}-\psi_{n-1, m}\right)\left(T_{n-1, m-1}+T_{n, m}\right)\right]
\end{array}
$$

or

$$
\begin{gathered}
J(\psi, T)=\frac{1}{12 d^{2}}\left[\left(\psi_{n-1, m-1}+\psi_{n-1, m}-\psi_{n, m-1}-\psi_{n, m}\right)\left(T_{n, m}+T_{n, m-1}\right)-\right. \\
\left(2 \psi_{n-1, m}-2 \psi_{n, m-1}+\psi_{n-1, m-1}-\psi_{n, m}\right)\left(T_{n, m}+T_{n-1, m}\right)-\quad(a .9) a \\
\left.\left(\psi_{n, m-1}-\psi_{n-1, m}\right)\left(T_{n-1, m-1}+T_{n, m}\right)\right]
\end{gathered}
$$




\section{References}

Anati, D., and H. Stommel, 1970. The initial phase of deep water formation in the northwest Mediterranean, during MEDOC '69, on the basis of observation made by "Atlantis II" January 25-February 12, 1969. Cahiers Océanographique 22: 343351.

Arakawa, A., 1966. Computational design for long-term numerical integration of the equations of fluid motion: two-dimensional incompressible flow. Part 1. Journal of Computational Physics 1: 119-143.

Armi, L., 1978. Some evidence for boundary mixing in the deep ocean. Journal of Geophysical Research 83: 1971-1979.

Beardsley, R. C., and J. F. Festa, 1972. A numerical model of convection driven by a surface stress and a non-uniform horizontal heating. Journal of Physical Oceanography 2: 444-455.

Boyle, E. A., and L. D. Keigwin, 1982. Deep circulation of the North Atlantic over the past 200000 years. Science 218: 784-787.

Brewer, P. G., W. S. Broecker, W. J. Jenkins, P. B. Rhines, C. G. Rooth, J. H. Swift, T. Takahashi, and R. W. Williams, 1983. A climatic freshening of the deep North Atlantic north of $50^{\circ} \mathrm{N}$ over the past 20 years. Science 222: 1237-1239.

Bryan, F., 1986. High-latitude salinity effects and interhemispheric thermohaline circulation. Nature 223: 301-304.

Bryan, K., and M. D. Cox, 1968. A nonlinear model of an ocean driven by wind and differential heating: Part I. Description of the three-dimensional velocity and density fields. Journal of the Atmospheric Sciences 25: 945-967.

Bryan, K., and L. J. Lewis, 1979. A water mass model of the world ocean. Journal of Geophysical Research 84: 2503-2517.

Bryden, H. L., and H. M. Stommel, 1984. Limiting processes that determine basic features of the circulation in the Mediterranean Sea. Oceanologica Acta 7: 289-296. 
Buneman, O., 1969. A compact non-iterative Poisson solver. SU-IPR 294: 1-11.

Busalacchi, A. J., and J. J. O'Brian, 1980. The seasonal variability in a model of the tropical Pacific. Journal of Physical Oceanography 10: 1929-1951.

Carmack, E. C., and K. Aagaard, 1973. On the deep water of the Greenland Sea. Deep Sea Research 20: 687-715.

Clarke, R. A., and J-C. Gascard, 1983. The formation of Labrador Sea Water, Part I. large scale processes. Journal of Physical Oceanography 13: 1764-1778.

Ellis, H., 1751. A letter to the Rev. Dr. Hales, F.R.S. from captain Henry Ellis, F.R.S. dated Jan. 7, 1750-1751. at Cape Monte Africa, ship Earl of Halifax. Philosophical Transaction of the Royal Society of London 47: 211-214.

Foster, T. D., and E. C. Carmack, 1976. Front zonal mixing and Antarctic bottom water formation in the southern Weddell Sea. Deep Sea Research 23: 301-317.

Gill, A. E., 1973. Circulation and bottom water formation in the Weddell Sea. Deep Sea Research 20: 111-140.

Gordon, A. L., 1978. Deep Antarctic convection west of Maud rise. Journal of Physical Oceanography 8: 600-612.

Gordon, A. L., 1982. Weddell deep water variability. Journal of Marine Research 40: Supplement 199-217.

Gordon, A. L., and P. Tchernia, 1972. Waters of the continental margin off the Adélie coast, Antarctica. In Antarctic Oceanology II: The Australian-New Zealand Sector, Antarctic Research Series 19: pp. 59-69. D. E. Hayes, ed., Washington D. C.

Held, M. I., and A. Y. Hou, 1980. Nonlinear axially symmetric circulation in a nearly inviscid atmosphere. Journal of the Atmospheric Sciences 37: 515-533.

Huang, R. X., 1984. The thermocline and current structure in subtropical/subpolar basins. Doctoral Dissertation. Woods Hole Oceanographic Institution and the Massachusetts Institute of Technology. 
Iselin, C. O'D., 1939. The influence of vertical and lateral turbulence on the characteristics of the waters at mid-depths. Transaction of the American Geophysical Union 20: 414-417.

Jacobs, S. S., A. F. Amos, and P. M. Bruckhausen, 1970. Ross Sea oceanography and Antarctic bottom water formation. Deep Sea Research 17: 935-962.

Joyce, T. M., and K. G. Speer, 1987. Modeling the large-scale influence of geothermal sources on abyssal flow. Journal of Geophysical Research 92: 2843-2850.

Killworth, P. D., 1976. The mixing and spreading phases of MEDOC. 1. Progress in Oceanography 7: 59-90.

Killworth, P.D., 1979. On "chimney" formation in the ocean. Journal of Physical Oceanography 9: 531-554.

Killworth, P. D., 1983. Deep convection in the world ocean. Reviews of Geophysics and Space Physics 21: 1-26.

Killworth, P. D., and P. C. Manins, 1980. A model of confined thermal convection driven by non-uniform heating from below. Journal of Fluid Mechanics 98: 587607.

Lacombe, H., and P. Tchernia, 1960. Quelques traits généraux de l'hydrologie Méditerranéenne d'après diverses campagnes hydrologiques rècentes en Méditerranée, dans le proche Atlantique et dans le détroit de Gibraltar. Cahiers Océanographique 12 année 8: 527-547.

Lazier, J. R. N., 1973. The renewal of Labrador Sea Water. Deep Sea Research 20: 341-353.

Luyten., J. R., J. Pedlosky, and H. Stommel, 1983. The ventilated thermocline. Journal of Physical Oceanography 13: 292-309.

Martinson, D. G., P. D. Killworth, and A. L. Gordon, 1981. A convective model for the Weddell polynya. Journal of Physical Oceanography 11: 466-488. 
McCartney, M. S., 1982. The subtropical circulation of mode waters. Journal of Marine Research 40: Supplement 427-464.

MEDOC Group, 1970. Observation of formation of deep water in the Mediterranean Sea, 1969. Nature 227: 1037-1040.

Morcos, S. A., 1972. Sources of Mediterranean Intermediate Water in the Levantine Sea. In Studies in physical oceanography, a tribute to Georg Wüst on his 80th birthday, A. L. Gordon, ed., Gordon and Bearch, New York, Vol. 2: 185-206

Moskalenko, L. V., and I. M. Ovchinnikov, 1965. Water masses of the Mediterranean Sea. In Basic features of the geological structure, hydrological regime, and biology of the Mediterranean. L. M. Fomin, ed. Translation from russian of the Institute for Modern Languages for the U.S.N. Oceanographic Office. pp. 202-218a.

Neumann, G., and W. J. Pierson, Jr., 1966. Principles of physical oceanography. Prentice-Hall, pp. 407, 545 pp.

Oort, A. H., and T. H. Vonder Haar, 1976. On the observed annual cycle in the ocean-atmosphere heat balance over the northern hemisphere. Journal of Physical Oceanography 6: 781-800.

Özsoy, E., M. A. Latif, and Ü. Ünlüata, 1981. On the formation of Levantine Intermediate water. Rapports Commission Internationale Mer Méditerranée 27(6): $51-65$.

Özturgut, F., 1976. The sources and spreading of the Levantine Intermediate water in the eastern Mediterranean. Saclant ASW Research Center Memorandum SM-92, La Spezia, Italy, $45 \mathrm{pp}$.

Philander, S. G. H., and R. C. Pacanowski, 1984. Simulations of the seasonal cycle in the tropical Atlantic Ocean . Geophysical Research Letters 11: 802-804.

Pollard, R. T., and S. Pu, 1985. Structure and circulation of the upper Atlantic Ocean north of the Azores. Proceedings in Oceanography 14: 443-462. 
Redi, H. M., 1982. Oceanic isopycnal mixing by coordinate rotation. Journal of Physical Oceanography 12: 1154-1158.

Roberts, G. O., 1977. Fast viscous convection. Journal of Geophysical and Astrophysical Fluid Dynamics 8: 197-233.

Robinson, A. R., and H. Stommel, 1959. The oceanic thermocline and the associated thermohaline circulation. Tellus 11: 295-308.

Roemmich, D., and C. Wunsch, 1984. Apparent changes in the climatic state of the deep North Atlantic ocean. Nature 307: 447-450.

Rossby, T. H., 1965. On thermal convection driven by non-uniform heating from below, an experimental study. Deep Sea Research 12: 9-16.

Ruddiman, W. F., and A. McIntyre, 1981. Oceanic mechanisms for the amplification of the 23000 year ice-volume cycle. Science 212: 617-627.

Said, M. A., 1986. Hydrographic characteristics and circulation of the Eastern Mediterranean waters. Report of the first POEM scientific workshop. to be published.

Sarmiento, J. L., and J. R. Toggweiler, 1984. A new model for the role of the oceans in determining atmospheric $\mathrm{P}_{\mathrm{CO}_{3}}$. Nature 308: 621-624.

Schneider, K. E., and R. S. Lindzen, 1977. Axially symmetric steady-state models of the basic state for instability and climate studies. Part I. Linearized calculations. Journal of the Atmospheric Sciences 34: 263-279.

Schneider, K. E., 1977. Axially symmetric steady-state models of the basic state for instability and climate studies. Part II. Nonlinear calculations. Journal of the Atmospheric Sciences 34: 280-296.

Schnitker, D., 1980. Quaternary deep-sea benthic foraminifers and bottom water masses. Annual Reviews of Earth Planetary Sciences 8: 343-370.

Sommerville, C. J. R., 1967. A nonlinear spectral model of convection in a fluid unevenly heated from below. Journal of the Atmospheric Sciences 24: 665-676. 
Stern, E. M., 1975. Ocean circulation physics. International geophysics series, Vol. 19: Academic Press, pp. 216-233, 246 pp.

Stommel, H., 1950. An example of thermal convection. Transaction of the American Geophysical Union 31: 553-554.

Stommel, H., 1962. On the smallness of sinking regions in the ocean. Proceedings of the National Academy of Science, U.S. 48: 776-772.

Stommel, H., 1972. Deep winter-time convection in the western Mediterranean Sea. In Studies in physical oceanography, a tribute to Georg Wüst on his 80th birthday, A. L. Gordon, ed., Gordon and Bearch, New York, Vol. 2: 207-218

Stommel, H., and G. Veronis, 1957. Steady convective motion in a horizontal layer of fluid heated uniformly from above and cooled non-uniformly from below. Tellus 9: 401-407.

Stone, P. H., 1968. Some properties of Hadley regimes on rotating and non-rotating planets. Journal of the Atmospheric Sciences 25: 644-657.

Veronis, G., 1969. On theoretical models of the thermocline circulation. Deep Sea Research 16: Supplement 301-323.

Warren, B. A., 1972. Insensitivity of subtropical mode water characteristics to meteorological fluctuations. Deep Sea Research 19: 1-19.

Welander, P., 1959. An advective model of the ocean thermocline. Tellus 11: 309318.

Wunsch, C., and B. Grant, 1982. Towards the general circulation of the North Atlantic Ocean. Progress in Oceanography 11: 1-59.

Wüst, G., 1935. The stratosphere of the Atlantic Ocean. Scientific results of the German Atlantic expedition of the research vessel Meteor 1925-1927. Vol. 6: Section 1. W. J. Emery, ed. Amerind publishing, 1978.

Wüst, G., 1961. On the vertical circulation of the Mediterranean Sea. Journal of Geophysical Research 66: 3261-3271. 